\title{
Well-posedness of the transport equation by stochastic perturbation
}

\author{
F. Flandoli ${ }^{1}$, M. Gubinelli ${ }^{2}$, E. Priola ${ }^{3}$ \\ (1) Dipartimento di Matematica Applicata "U. Dini", Università di Pisa, Italia \\ (2) CEREMADE (UMR 7534), Université Paris Dauphine, France
}

(3) Dipartimento di Matematica, Università di Torino, Italia

May 29, 2018

\begin{abstract}
We consider the linear transport equation with a globally Hölder continuous and bounded vector field, with an integrability condition on the divergence. While uniqueness may fail for the deterministic PDE, we prove that a multiplicative stochastic perturbation of Brownian type is enough to render the equation well-posed. This seems to be the first explicit example of partial differential equation that become well-posed under the influence of noise. The key tool is a differentiable stochastic flow constructed and analyzed by means of a special transformation of the drift of Itô-Tanaka type.
\end{abstract}

\section{Introduction}

The transport equation in $[0, T] \times \mathbb{R}^{d}$

$$
\begin{aligned}
\partial_{t} u(t, x)+b(t, x) \cdot D u(t, x) & =0, \quad(t, x) \in[0, T] \times \mathbb{R}^{d}, \\
u(0, x) & =u_{0}(x), \quad x \in \mathbb{R}^{d}
\end{aligned}
$$

driven by a vector field $b:[0, T] \times \mathbb{R}^{d} \rightarrow \mathbb{R}^{d}$ which is of class $L^{1}\left(0, T ; W_{l o c}^{1, \infty}\left(\mathbb{R}^{d}, \mathbb{R}^{d}\right)\right)$ with a linear growth condition in $x$, can be solved in several classical ways and in different function spaces. A remarkable extension has been obtained by R.J. Di Perna and P.L. Lions [10] who proved, under the assumption $b \in L^{1}\left(0, T ; W_{\text {loc }}^{1,1}\left(\mathbb{R}^{d}, \mathbb{R}^{d}\right)\right)$ with a linear growth condition and $\operatorname{div} b \in L^{1}\left(0, T ; L^{\infty}\left(\mathbb{R}^{d}\right)\right)$, that a unique $L^{\infty}\left([0, T] \times \mathbb{R}^{d}\right)$ solution exists, weak-* continuous in time, for any given $u_{0} \in L^{\infty}\left(\mathbb{R}^{d}\right)$. Moreover, a generalized notion of flow is introduced and its existence and uniqueness is proved. L. Ambrosio [2] proved that uniqueness of $L^{\infty}$ solutions is still true when $W_{l o c}^{1,1}\left(\mathbb{R}^{d}, \mathbb{R}^{d}\right)$ is replaced by $B V_{l o c}\left(\mathbb{R}^{d}, \mathbb{R}^{d}\right)$; furthermore he showed that a sufficient condition for the uniqueness of the generalized flow is that the negative part of $\operatorname{div} b$ is $L^{1}\left(0, T ; L^{\infty}\left(\mathbb{R}^{d}\right)\right)$. The literature following [10] is wide, see a partial review in [3]. See also generalizations to transport-diffusion equations and the associated stochastic differential equations by C. Le Bris and P.L. Lions [27] and A. Figalli [12] (in a direction different from the one of the present paper).

Under weaker conditions on $b$, there are examples of non-uniqueness; see Section 6.1. The aim of this paper is to show that, under a suitable random perturbation, $L^{\infty}$-solutions are unique, 
even in the case of vector fields $b$ such that the deterministic Cauchy problem for the transport equation may have multiple solutions. This result is obtained by introducing a multiplicative white noise in the PDE. Precisely, we consider the stochastic PDE (SPDE)

$$
\begin{aligned}
& d_{t} u(t, x)+(b(t, x) \cdot D u(t, x)) d t+\sum_{i=1}^{d} e_{i} \cdot D u(t, x) \circ d W_{t}^{i}=0, \\
& u(0, x)=u_{0}(x), \quad x \in \mathbb{R}^{d}
\end{aligned}
$$

where $e_{1}, \ldots, e_{d}$ is the canonical basis of $\mathbb{R}^{d}$ and $W_{t}=\left(W_{t}^{1}, \ldots, W_{t}^{d}\right)$ is a standard Brownian motion in $\mathbb{R}^{d}$. The stochastic integration is understood in the Stratonovich sense.

We study existence and uniqueness of $L^{\infty}$-solutions, strong in the probabilistic sense, when $b$ is measurable, bounded, globally $\alpha$-Hölder continuous in space uniformly in time, for some $\alpha \in(0,1)$ and $\operatorname{div} b \in L_{\text {loc }}^{1}\left([0, T] \times \mathbb{R}^{d}\right)$. In particular, we prove that uniqueness always holds in dimension $d=1$ and in any dimension $d \geq 1$ when $\alpha \in(1 / 2,1)$. If $\alpha \in(0,1 / 2]$ we still get uniqueness assuming in addition a global integrability condition on div $b$, i.e.,

$$
\operatorname{div} b \in L^{p}\left([0, T] \times \mathbb{R}^{d}\right)
$$

for some $p>2$ (recall that a global integrability on $\operatorname{div} b$ different from (2) is also needed in the deterministic case to get uniqueness; see [10, 2]).

Moreover, we have existence and uniqueness of $B V_{l o c}$-solutions, when $u_{0} \in B V_{l o c}$, assuming only the Hölder condition on $b$, without any assumption on $\operatorname{div} b$.

Our result gives the first concrete example of partial differential equation that may lack uniqueness without noise, but is well-posed with a suitable noise. This phenomenon is well studied for ordinary differential equations but it is one of the more interesting direction of investigations in the theory of SPDEs with the ultimate aim of proving the well-posedness of suitable stochastic perturbations of relevant fluid-dynamics equations. Most attempts in this direction, until now, focused on additive noise perturbations, see a discussion in [1]. Regularization by multiplicative noise is, as far as we know, a new phenomenon.

The choice of Stratonovich integral in eq. (1) is motivated by two related facts. On one side (see H. Kunita 24]), for smooth data and regular vector field $b$, eq. (11) has an explicit solution $u(t, x)=u_{0}\left(\phi_{t}^{-1}(x)\right)$ where $\phi_{t}(x)$ is the flow map giving the unique strong solution $\left(X_{t}^{x}\right)_{t \geq 0}$ of the SDE

$$
d X_{t}^{x}=b\left(t, X_{t}^{x}\right) d t+d W_{t}, \quad t \geq 0, \quad X_{0}^{x}=x .
$$

On the other side, Stratonovich integral is motivated by the Wong-Zakai principle. Roughly, it state that differential equations driven by regular random functions usually converge to the Stratonovich version of the limiting stochastic differential equations provided that these random functions tend to Brownian motion. In Appendix C we will prove two versions of this principle for the stochastic transport equation.

Existence of $L^{\infty}$-solutions to (1) does not require all the assumptions stated above on $b$ since a compactness argument require only that $b \in L_{\text {loc }}^{1}\left([0, T] \times \mathbb{R}^{d} ; \mathbb{R}^{d}\right)$ and $\operatorname{div} b \in L_{\text {loc }}^{1}\left([0, T] \times \mathbb{R}^{d}\right)$.

As in the deterministic case (see [10, 2]), uniqueness of $L^{\infty}$-weak solutions is related to some form of commutator lemma which allows to perform differential computations on regularizations of $L^{\infty}$-solutions. In the deterministic case one has strong convergence to zero of the commutator. Here we have strong convergence in $L_{l o c}^{1}$ only for $d=1$, since (2) implies that $\operatorname{div} b=D b \in$ $L_{l o c}^{1}\left([0, T] \times \mathbb{R}^{d}\right)$. For $d>1$ our conditions on $b$ do not guarantee the strong convergence of 
the commutator and we are forced to exploit some non-trivial regularization properties of the stochastic characteristic equation which come from the non-degeneracy of the noise. Let us briefly explain this phenomenon.

Observe that formally the mean value $\bar{u}(t, x)=\mathbb{E}[u(t, x)]$ of the solution to eq. (11) satisfies

$$
\begin{aligned}
& \frac{d}{d t} \bar{u}(t, x)+b(t, x) \cdot D \bar{u}(t, x)+\frac{1}{2} \Delta \bar{u}(t, x) d t=0, \\
& \bar{u}(0, x)=u_{0}(x), \quad x \in \mathbb{R}^{d},
\end{aligned}
$$

(this may be explained by Lemma 13). The regularizing effect of the viscous term is linked with the regularity properties of the law of the diffusion $X_{t}^{x}$. At the path-wise level no regularization of $u(t, x)$ can appear (as witnessed by the characteristics method). However the non-degeneracy of the diffusion has a remarkable effect also on integrals of the form

$$
\int_{0}^{T} f\left(s, X_{s}^{x}\right) d s
$$

where $f:[0, T] \times \mathbb{R}^{d} \rightarrow \mathbb{R}^{d}$ is a deterministic but possibly time-dependent function. The occupation measure of a typical trajectory of $X^{x}$ (by occupation measure we mean the pushforward of Lebesgue measure on $[0, T]$ under the map $\left.t \mapsto X_{t}^{x}\right)$ has a density with respect to Lebesgue measure in dimension one, the local time, see 33. In dimension larger than one its regularity is less easy, but in any dimension it may be captured by means of Itô formula and the regularity of solutions of an auxiliary parabolic equations. Indeed, if we consider a solution $F$ to the parabolic PDE

$$
\partial_{t} F+\frac{1}{2} \Delta F+b \cdot D F=f
$$

on $[0, T] \times \mathbb{R}^{d}$, then an application of the Itô formula allows us to rewrite the above integral as

$$
\int_{0}^{T} f\left(s, X_{s}^{x}\right) d s=F\left(T, X_{T}^{x}\right)-F(0, x)-\int_{0}^{T} D F\left(s, X_{s}^{x}\right) \cdot d W_{s} .
$$

The point of this manouvre (which we will call the "Itô-Tanaka trick") is to replace the timeaverage over the diffusion path by a combination of terms which usually are better behaved than the l.h.s.. Indeed, under appropriate conditions, the non-degeneracy of the diffusion implies that the solution $F$ of the parabolic PDE is more regular than the original function $f$. In some sense the Itô-Tanaka trick allows us to partly transfer the parabolic regularization of the law of the diffusion to its sample paths.

This basic strategy is our key tool. It gives us the following two properties of the characteristics equation (3):

(i) Under the assumption that $b(t, \cdot) \in C_{b}^{\alpha}\left(\mathbb{R}^{d} ; \mathbb{R}^{d}\right)$ uniformly in time, equation (3) generates a stochastic flow of $C^{1+\alpha^{\prime}}$-diffeomorphisms $\phi_{t}(x, \omega)$, for any $0<\alpha^{\prime}<\alpha$. Moreover this flow is stable under approximation of the vector field. Related results in $d=1$ have been proved in [13].

(ii) Under the integrability assumption (2) on $\operatorname{div} b$, we show that the Jacobian $J \phi_{t}(\cdot, \omega)$ of the flow is in $L^{2}\left(0, T ; W_{l o c}^{1,2}\right) P$-a.s. (see Theorem 11). Note that, since the noise is additive, the Jacobian $J \phi_{t}(x, \omega)$ solves pathwise at least formally the deterministic ODE

$$
\frac{d}{d t} J \phi_{t}(x, \omega)=\operatorname{div} b\left(t, \phi_{t}(x, \omega)\right) J \phi_{t}(x, \omega)
$$


and thus

$$
\log J \phi_{t}(x, \omega)=\int_{0}^{t} \operatorname{div} b\left(s, \phi_{s}(x, \omega)\right) d s .
$$

In the deterministic case there is no hope to differentiate in $x$ without further differentiability assumptions on $\operatorname{div} b$, but in the stochastic case we use again the improved regularity of integrals of the form (5) thanks to the Itô-Tanaka trick and to some classical $L^{p}$-parabolic regularity results. The required integrability condition on $\operatorname{div} b$ (see (2) ) turns out to be different from the one imposed in the deterministic setting [10, 2].

When $\alpha \in(1 / 2,1)$, we can prove distributional convergence of the commutator (and so uniqueness for our SPDE) by combining the Hölder regularity of $b$ and the one of the stochastic flow (so using only (i)). In the case of $\alpha \in(0,1 / 2]$ we can still prove distributional convergence of the commutator, but we need both (i) and (ii) (and so we require (2)).

The differentiability of the flow gives easily existence and uniqueness of $B V_{\text {loc }}$ solutions (and other more regular spaces) to the SPDE without any requirement on the divergence (see Appendix (A).

A remark on the connections with the work of Y. Le Jan and O. Raimond 28] on generalized stochastic flows is important. At present, no precise comparison can be made between the result of the present work and those of [28], but it is clear that [28] has been a source of inspiration for us, like [2, 10].

Conceptual similarities between all these works can be seen in the following results of [28]. It deals with a stochastic transport-like equation, or to be more precise, a stochastic continuity equation written in weak form and a variation-of-constant reformulation of it, see equations (e) and (d) respectively of Theorem 3.2. An existence and uniqueness result is proved, in a special class of solutions, under very general assumptions. Finally, 28] gives criteria for existence of an associated flow of maps, or on the contrary for the possibility of coalescence and diffusion. The general results are applied to examples where the coefficients have a very poor Sobolev regularity.

What is entirely different between our work and [28] is that 28] deals with stochastic equations which are well posed in the weak sense (martingale sense) but not necessarily strongly well posed. The relevant examples, at present, are constructed by means of suitable diffusion coefficients (and infinite dimensional noise, often, like the isotropic Brownian motion); the drift part does not play a relevant role. The poor regularity of coefficients mentioned above regards the diffusion coefficients. On the contrary, our purpose is to deal with a non-regular drift coefficient (and we choose a trivial but non-degenerate diffusion part for sake of simplicity), following the philosophy that we randomly perturb a deterministic transport equation having non-regular drift. Our stochastic equations are also strongly well posed and they always define a stochastic flow of diffeomorphisms.

Finding a synthesis of these different approaches to non-regular transport (or continuity) equations, deterministic and stochastic, would be a very interesting progress. Let us end this introduction by mentioning a few other open questions.

The generalization to nonlinear transport equations, where $b$ depends on $u$ itself, would be a major next step for applications to fluid dynamics but it turns out to be a difficult problem. Specifically there are already some difficulties in dealing with a vector field $b$ which depends itself on the random perturbation $W$. There is no obvious extension of the Itô-Tanaka trick to integrals of the form $\int_{0}^{T} f\left(\omega, s, X_{s}^{x}(\omega)\right) d s$ with random $f$. As we will show in Section [6, it 
is very easy to produce examples, both for the linear SPDE (11) and for a stochastic version of Euler equation which show that the particular noise we use does not have any regularizing effect in this case. Thus new ideas are needed to approach nonlinear problems.

The linear case with deterministic $b$ still contains interesting open problems. N. Depauw [9] gave examples of non-uniqueness in $d=2$ for divergence free bounded measurable fields $b$ with a condition on the bounded variation norm. Our results do not cover this case. In particular, the existence of stochastic flows under $L^{\infty}$ assumptions on $b$ is an interesting open problem.

Finally, in Section 6 we see that the classical one dimensional example with $b(x) \simeq|x|^{\gamma}$, $\gamma \in(0,1)$, is covered by our uniqueness result. The differentiablility of the stochastic flow $\phi$ means in particular that its stretching $J \phi$ around $x=0$ is very large but finite. However, we can prove $J \phi \in L^{2}\left(0, T ; W_{\text {loc }}^{1,2}\right)$ only for $\gamma \in(1 / 2,1)$. It is not clear if $\gamma \geq 1 / 2$ is a natural threshold for the smoothness of the stretching or it is just a limitation of our approach.

Acknowledgement. The authors would like to thank the anonymous referees for the careful reading of the first version of this paper and for their remarks which helped to greatly improve the paper.

Plan. The main body of the paper is devoted to the analysis of weak $L^{\infty}$-solutions and preliminaries on stochastic flows. In Sect. 2 we prove the existence of a global stochastic flow associated to eq. (3) and its differentiability properties. Sect. 3 is devoted to prove that under our hypotheses on $\operatorname{div} b$ the Jacobian of the flow is in $L^{2}\left(0, T ; W_{\mathrm{loc}}^{1,2}\left(\mathbb{R}^{d}\right)\right)$. In Sect. 4 we prove existence of weak solutions to the SPDE (11). Sect. 5 is devoted to prove uniqueness of $L^{\infty}$-solutions to the SPDE (1). Finally, in Sect. 6 we collect some positive and negative examples.

Then we present a number of appendixes on related results. Appendix $\mathrm{A}$ is devoted to existence and uniqueness of $B V_{l o c}$ solutions, Appendix B to an equivalent pathwise formulation of the SPDE, Appendix C gives Wong-Zakai approximation results finally Appendix D gives uniqueness results by fractional Sobolev spaces non covered in the main text.

Notations. Usually we denote by $D_{i} f$ the derivative in the $i$-th coordinate direction and with $\left(e_{i}\right)_{i=1, \ldots, d}$ the canonical basis of $\mathbb{R}^{d}$ so that $D_{i} f=e_{i} \cdot D f$. For partial derivatives of any order $n \geq 1$ we use the notation $D_{i_{1}, \ldots, i_{n}}^{n}$. If $\eta: \mathbb{R}^{d} \rightarrow \mathbb{R}^{d}$ is a $C^{1}$-diffeomorphism we will denote by $J \eta(x)=\operatorname{det}[D \eta(x)]$ its Jacobian determinant. For a given function $f$ depending on $t \in[0, T]$ and $x \in \mathbb{R}^{d}$, we will also adopt the notation $f_{t}(x)=f(t, x)$.

Let $T>0$ be fixed. For $\alpha \in(0,1)$ define the space $L^{\infty}\left(0, T ; C_{b}^{\alpha}\left(\mathbb{R}^{d}\right)\right)$ as the set of all bounded Borel functions $f:[0, T] \times \mathbb{R}^{d} \rightarrow \mathbb{R}$ for which

$$
[f]_{\alpha, T}=\sup _{t \in[0, T]} \sup _{x \neq y \in \mathbb{R}^{d}} \frac{|f(t, x)-f(t, y)|}{|x-y|^{\alpha}}<\infty
$$

$\left(|\cdot|\right.$ denotes the Euclidean norm in $\mathbb{R}^{d}$ for every $d$, if no confusion may arise). This is a Banach space with respect to the usual norm $\|f\|_{\alpha, T}=\|f\|_{0}+[f]_{\alpha, T}$ where $\|f\|_{0}=\sup _{(t, x) \in[0, T] \times \mathbb{R}^{d}}|f(t, x)|$.

We write $L^{\infty}\left(0, T ; C_{b}^{\alpha}\left(\mathbb{R}^{d} ; \mathbb{R}^{d}\right)\right)$ for the space of all vector fields $f:[0, T] \times \mathbb{R}^{d} \rightarrow \mathbb{R}^{d}$ having all components in $L^{\infty}\left(0, T ; C_{b}^{\alpha}\left(\mathbb{R}^{d}\right)\right)$.

Moreover, for $n \geq 1, f \in L^{\infty}\left(0, T ; C_{b}^{n+\alpha}\left(\mathbb{R}^{d}\right)\right)$ if all spatial partial derivatives $D_{i_{1}, \ldots, i_{k}}^{k} f \in$ 
$L^{\infty}\left(0, T ; C_{b}^{\alpha}\left(\mathbb{R}^{d}\right)\right)$, for all orders $k=0,1, \ldots, n$. Define the corresponding norm as

$$
\|f\|_{n+\alpha, T}=\|f\|_{0}+\sum_{k=1}^{n}\left\|D^{k} f\right\|_{0}+\left[D^{n} f\right]_{\alpha, T}
$$

where we extend the previous notations $\|\cdot\|_{0}$ and $[\cdot]_{\alpha, T}$ to tensors. The definition of the space $L^{\infty}\left(0, T ; C_{b}^{n+\alpha}\left(\mathbb{R}^{d} ; \mathbb{R}^{d}\right)\right)$ is similar. The previous functions spaces can be defined similarly when $T=+\infty$ (i.e., we are considering functions defined on $\left.[0, \infty) \times \mathbb{R}^{d}\right)$. The spaces $C_{b}^{n+\alpha}\left(\mathbb{R}^{d}\right)$ and $C_{b}^{n+\alpha}\left(\mathbb{R}^{d} ; \mathbb{R}^{d}\right)$ are defined as before but only involve functions $f: \mathbb{R}^{d} \rightarrow \mathbb{R}^{d}$ which do not depend on time. Moreover, we say that $f: \mathbb{R}^{d} \rightarrow \mathbb{R}^{d}$ belongs to $C^{n, \alpha}, n \in \mathbb{N}, \alpha \in(0,1)$, if $f$ is continuous on $\mathbb{R}^{d}, n$-times differentiable with all continuous derivatives and the derivatives of order $n$ are locally $\alpha$-Hölder continuous. Finally, $C_{0}^{0}\left(\mathbb{R}^{d}\right)$ denotes the space of all real continuous functions defined on $\mathbb{R}^{d}$, having compact support and by $C_{0}^{\infty}\left(\mathbb{R}^{d}\right)$ its subspace consisting of infinitely differentiable functions.

For any $r>0$ we denote by $B(r)$ the Euclidean ball centered in 0 of radius $r$ and by $C_{r}^{\infty}\left(\mathbb{R}^{d}\right)$ the space of smooth functions with compact support in $B(r)$; moreover, $\|\cdot\|_{L_{r}^{p}}$ and $\|\cdot\|_{W_{r}^{1, p}}$ stand for, respectively, the $L^{p}$-norm and the $W^{1, p}$-norm on $B(r), p \in[1, \infty]$. We let also $[f]_{C_{r}^{\theta}}=\sup _{x \neq y \in B(r)}|f(x)-f(y)| /|x-y|^{\theta}$.

We will often use the standard mollifiers. Let $\vartheta: \mathbb{R}^{d} \rightarrow \mathbb{R}$ be a smooth test function such that $0 \leq \vartheta(x) \leq 1, x \in \mathbb{R}^{d}, \vartheta(x)=\vartheta(-x), \int_{\mathbb{R}^{d}} \vartheta(x) d x=1, \operatorname{supp}(\vartheta) \subset B(2), \vartheta(x)=1$ when $x \in B(1)$. For any $\varepsilon>0$, let $\vartheta_{\varepsilon}(x)=\varepsilon^{-d} \vartheta(x / \varepsilon)$ and for any distribution $g: \mathbb{R}^{d} \rightarrow \mathbb{R}^{n}$ we define the mollified approximation $g^{\varepsilon}$ as

$$
g^{\varepsilon}(x)=\vartheta_{\varepsilon} * g(x)=g\left(\vartheta_{\varepsilon}(x-\cdot)\right), \quad x \in \mathbb{R}^{d} .
$$

If $g$ depends also on time $t$, we consider $g^{\varepsilon}(t, x)=\left(\vartheta_{\varepsilon} * g(t, \cdot)\right)(x), t \in[0, T], x \in \mathbb{R}^{d}$.

Recall that, for any smooth bounded domain $\mathcal{D}$ of $\mathbb{R}^{d}$, we have: $f \in W^{\theta, p}(\mathcal{D}), \theta \in(0,1)$, $p \geq 1$, if and only if $f \in L^{p}(\mathcal{D})$ and

$$
[f]_{W^{\theta, p}}^{p}=\iint_{\mathcal{D} \times \mathcal{D}} \frac{|f(x)-f(y)|^{p}}{|x-y|^{\theta p+d}} d x d y<\infty .
$$

We have $W^{1, p}(\mathcal{D}) \subset W^{\theta, p}(\mathcal{D}), \theta \in(0,1)$.

Throughout the paper we will assume a stochastic basis with a $d$-dimensional Brownian motion $\left(\Omega,\left(\mathcal{F}_{t}\right), \mathcal{F}, P,\left(W_{t}\right)\right)$ to be given. We denote by $\mathcal{F}_{s, t}$ the completed $\sigma$-algebra generated by $W_{u}-W_{r}, s \leq r \leq u \leq t$, for each $0 \leq s<t$.

\section{Differentiable stochastic flow with $C_{b}^{\alpha}$ drift}

Given $s \in[0, T]$ and $x \in \mathbb{R}^{d}$, consider the stochastic differential equation in $\mathbb{R}^{d}$ :

$$
d X_{t}=b\left(t, X_{t}\right) d t+d W_{t}, \quad t \in[s, T], \quad X_{s}=x .
$$

A classical fact is the existence and uniqueness of a weak solution, obtained for instance by Girsanov transform. Yu. Veretennikov [36] proved that boundedness of $b$ (uniformly in $t$ ) is enough to have path-wise uniqueness and existence of strong probabilistic solutions. For related works 
see also the more recent paper [21] by N.V. Krylov and M. Röckner where strong uniqueness is proved under some integrability assumption on $b$. These works are based on the technique introduced by Zvonkin [38] of removing the irregular drift by a suitable change of coordinates in the SDE. The fact that such a coordinate change modifies the drift is a consequence of the Itô formula. Technically, as we will see in the proof of Th. 5, the Itô-Tanaka trick is similar to the Zvonkin approach. It is worthwhile to note however that the heuristic behind is not necessarily the same and in our opinion the Itô-Tanaka point of view has wider range of applicability (as we demonstrate in the control of the Jacobian of the flow).

A related interesting result has been obtained by A.M. Davie in [8]. Under the assumption that $b$ is measurable and bounded, he proved that the (deterministic) integral equation

$$
x(t)=x+\int_{0}^{t} b(s, x(s)) d s+w(t)
$$

has a unique solution $x(\cdot) \in C\left(0, T ; \mathbb{R}^{d}\right)$ for all $w \in N^{c}$ where $N \subset C\left(0, T ; \mathbb{R}^{d}\right)$ is a set which has probability zero according to Wiener measure. This paper contains also the very interesting key estimate

$$
E\left[\left|\int_{0}^{1}\left(b\left(s, x+W_{s}\right)-b\left(s, W_{s}\right)\right) d s\right|^{p}\right] \leq C_{p}\|b\|_{\infty}|x|^{p}, \quad x \in \mathbb{R}^{d}
$$

where $C_{p}$ is an absolute constant not depending on $b$. This estimate is obtained by non-trivial direct computations and show very explicitly the regularization phenomenon which occurs when considering average values of functions along the trajectories of diffusions (Brownian motion in this case).

In all the cited works the analysis of the flows is however missing, essentially they deal only with (various forms of) path-wise uniqueness of the SDE. For papers that tackle existence of global flows of homeomorphisms for SDEs without global Lipschitz coefficients see [37, 11] and the references therein. However, the assumptions of these works are too strong for our purposes.

Our key result is the existence of a differentiable stochastic flow $(x, s, t) \mapsto \phi_{s, t}(x)$ for equation (7) under the following hypothesis:

Hypothesis 1 There exists $\alpha \in(0,1)$ such that $b \in L^{\infty}\left(0, T ; C_{b}^{\alpha}\left(\mathbb{R}^{d} ; \mathbb{R}^{d}\right)\right)$.

Recall the relevant definition from [23]:

Definition 1 A stochastic flow of diffeomorphisms (resp. of class $\left.C^{1, \alpha}\right)$ on $\left(\Omega,\left(\mathcal{F}_{t}\right), \mathcal{F}, P,\left(W_{t}\right)\right.$ ) associated to equation (7) is a map $(s, t, x, \omega) \mapsto \phi_{s, t}(x)(\omega)$, defined for $0 \leq s \leq t \leq T, x \in \mathbb{R}^{d}$, $\omega \in \Omega$ with values in $\mathbb{R}^{d}$, such that

(a) given any $s \in[0, T], x \in \mathbb{R}^{d}$, the process $X^{s, x}=\left(X_{t}^{s, x}, t \in[s, T]\right)$ defined as $X_{t}^{s, x}=\phi_{s, t}(x)$ is a continuous $\mathcal{F}_{s, t}$-measurable solution of equation (7),

(b) P-a.s., $\phi_{s, t}$ is a diffeomorphism, for all $0 \leq s \leq t \leq T$, and the functions $\phi_{s, t}(x), \phi_{s, t}^{-1}(x)$, $D \phi_{s, t}(x), D \phi_{s, t}^{-1}(x)$ are continuous in $(s, t, x)$ (resp. of class $C^{\alpha}$ in $x$ uniformly in $(s, t)$ ),

(c) P-a.s., $\phi_{s, t}(x)=\phi_{u, t}\left(\phi_{s, u}(x)\right)$ for all $0 \leq s \leq u \leq t \leq T$ and $x \in \mathbb{R}^{d}$ and $\phi_{s, s}(x)=x$. 
As already mentioned, the main ingredient to obtain the regularity of the flow is the observation that the time integral $\int_{0}^{t} b\left(s, X_{s}^{x}\right) d s$ has richer regularity properties than expected only on the basis of the regularity of $b$. To reveal them we have to use the regularity theory of parabolic PDEs. Let $\lambda>0$ be fixed. Let us extend $b$ to the whole $[0, \infty) \times \mathbb{R}^{d}$ by setting

$$
b(t, x)=b(T, x), \quad t \geq T, \quad x \in \mathbb{R}^{d} .
$$

Clearly we have that $b \in L^{\infty}\left(0, \infty ; C_{b}^{\alpha}\left(\mathbb{R}^{d} ; \mathbb{R}^{d}\right)\right)$. Given a function $f \in L^{\infty}\left(0, \infty ; C_{b}^{\alpha}\left(\mathbb{R}^{d} ; \mathbb{R}^{d}\right)\right)$, consider the following backward parabolic system (collecting $d$ independent equations):

$$
\partial_{t} u_{\lambda}+L^{b} u_{\lambda}-\lambda u_{\lambda}=f, \quad(t, x) \in[0, \infty) \times \mathbb{R}^{d},
$$

where

$$
L^{b} u=\frac{1}{2} \Delta u+b \cdot D u
$$

and $u:[0,+\infty) \times \mathbb{R}^{d} \rightarrow \mathbb{R}^{d}$ (eq. (10) has to be interpreted componentwise).

Since $b$ and $f$ are only measurable in time instead of continuous, the notion of solution to (9) is not standard. We follow [20] by prescribing that a function $u:[0,+\infty) \times \mathbb{R}^{d} \rightarrow \mathbb{R}^{d}$ which belongs to $L^{\infty}\left(0, \infty ; C_{b}^{2+\alpha}\left(\mathbb{R}^{d} ; \mathbb{R}^{d}\right)\right)$ is a solution to (9) if

$$
u(t, x)-u(s, x)=\int_{s}^{t}\left[-L^{b} u(r, x)+\lambda u(r, x)+f(r, x)\right] d r
$$

for every $t \geq s \geq 0, x \in \mathbb{R}^{d}$. From this identity it follows that $u(\cdot, x)$ is Lipschitz continuous for every $x \in \mathbb{R}^{d}$. Other regularity properties can be found in [20].

The next result deals with Schauder estimates and is known even in a more general form (see [20] and the references therein). A-priori estimates of the type (11) were first proved in [4]. We will only sketch the proof and refer to [20] for more details. The backward equation (9) is not supplemented by the value of the limit $u(\infty, x)$ and uniqueness is due to the condition of uniform boundedness of $u$.

Theorem 2 Let us consider equation (9) with $b, f \in L^{\infty}\left(0, \infty ; C_{b}^{\alpha}\left(\mathbb{R}^{d} ; \mathbb{R}^{d}\right)\right)$. Then there exists a unique solution $u=u_{\lambda}$ to equation (9) in the space $L^{\infty}\left(0, \infty ; C_{b}^{2+\alpha}\left(\mathbb{R}^{d} ; \mathbb{R}^{d}\right)\right)$. Moreover there exists $C>0$ (independent on $u$ and $f$ ) such that

$$
\sup _{t \geq 0}\|u(t, \cdot)\|_{C_{b}^{2+\alpha}\left(\mathbb{R}^{d} ; \mathbb{R}^{d}\right)} \leq C \sup _{t \geq 0}\|f(t, \cdot)\|_{C_{b}^{\alpha}\left(\mathbb{R}^{d} ; \mathbb{R}^{d}\right)}
$$

Proof. Step 1 (uniqueness). Uniqueness follows from the maximum principle $\|u\|_{0} \leq$ $\lambda^{-1}\|f\|_{0}$ applied to the difference of two solutions. The maximum principle under our conditions is proved in [20, Theorem 4.1] (the proof is more delicate than in the classical case when $b$ and $f$ are continuous in $t$, see [17, Theorem 8.1.7]). For completeness we give also a self-contained probabilistic proof. From [36] or 21], under Hypothesis 1 there exists a unique strong solution $\left(X_{t}^{s, x}\right)_{t \geq 0}$ of equation (7). Let $u \in L^{\infty}\left(0, \infty ; C_{b}^{2+\alpha}\left(\mathbb{R}^{d} ; \mathbb{R}^{d}\right)\right)$ be a solution of equation (9). For any given $s$, we may apply Itô formula to $e^{-\lambda(t-s)} u\left(t, X_{t}^{s, x}\right)$ (see Lemma 3 below) in the $t$ variable. Taking then expectation we get

$$
e^{-\lambda(t-s)} E\left[u\left(t, X_{s, t}^{x}\right)\right]=u(s, x)+\int_{s}^{t} e^{-\lambda(r-s)} E\left[f\left(r, X_{s, r}^{x}\right)\right] d r .
$$


As $t \rightarrow \infty$ (recall that $u$ is bounded) we obtain

$$
u(s, x)=-\int_{s}^{\infty} e^{-\lambda(r-s)} E\left[f\left(r, X_{s, r}^{x}\right)\right] d r .
$$

Then $u$ is uniquely determined by $f$ and $b$. This also gives the estimate $\|u\|_{0} \leq \lambda^{-1}\|f\|_{0}$ mentioned above.

Step 2 (existence and estimate (11)). We only recall the idea of the proof, see [20] for details. If $b=0$, then the result follows by using the explicit formula

$$
u(t, x)=\int_{t}^{+\infty} e^{-\lambda(r-t)} P_{r-t} f(r, \cdot)(x) d r=\int_{0}^{+\infty} e^{-\lambda s} P_{s} f(t+s, \cdot)(x) d s
$$

(where $\left(P_{t}\right)$ denotes the forward heat semigroup) and well known estimates on the spatial derivatives of $P_{t} g$ when $g \in C_{b}^{\alpha}\left(\mathbb{R}^{d}\right)$ and $t>0$.

In the general case, using to the boundedness of $b$ and the maximum principle, we get easily a-priori estimates for equation (9) (assuming that there exists a bounded solution $u$ ). Then a continuity method (see [20, Lemma 4.3]) allows to get the existence of the solution which verifies equation (9), along with the estimate (11).

In the previous proof we have used Itô formula for solutions of equation (9), although their regularity in time is not standard for Itô formula. We give a self-contained proof of the validity of Itô formula in our hypotheses since we have to use it again below in the essential step of the change of variables from the SDE (7) to the SDE (19).

Lemma 3 Let $u:[0,+\infty) \times \mathbb{R}^{d} \rightarrow \mathbb{R}$ be a function of class $L^{\infty}\left(0, \infty ; C_{b}^{2+\alpha}\left(\mathbb{R}^{d}\right)\right)$, such that

$$
U(t, x)-U(s, x)=\int_{s}^{t} V(r, x) d r
$$

for every $t \geq s \geq 0, x \in \mathbb{R}^{d}$, with $V \in L^{\infty}\left(0, \infty ; C_{b}^{\alpha}\left(\mathbb{R}^{d}\right)\right)$. Let $\left(X_{t}\right)_{t \geq 0}$ be a continuous adapted process of the form

$$
X_{t}=X_{0}+\int_{0}^{t} b_{s} d s+\int_{0}^{t} \sigma_{s} d W_{s}
$$

where $b$ and $\sigma$ are (resp. $\mathbb{R}^{d}$-valued and $\mathbb{R}^{d \times d}$-valued) progressively measurable processes, $b$ integrable and $\sigma$ square integrable in $t$ with probability one. Then

$$
\begin{aligned}
U\left(t, X_{t}\right)=U(0, x)+ & \int_{0}^{t}\left(V+b_{s} \cdot D U+\frac{1}{2} \operatorname{Tr}\left(\sigma \sigma^{T} D^{2} U\right)\right)\left(s, X_{s}\right) d s \\
& +\int_{0}^{t}\left\langle D U\left(s, X_{s}\right), \sigma_{s} d W_{s}\right\rangle .
\end{aligned}
$$

Proof. Set

$$
U_{\varepsilon}(t, x)=\varepsilon^{-1} \int_{t}^{t+\varepsilon} U(s, x) d s, \quad V_{\varepsilon}(t, x)=\varepsilon^{-1} \int_{t}^{t+\varepsilon} V(s, x) d s .
$$

The time derivative (see (13) )

$$
\partial_{t} U_{\varepsilon}=\varepsilon^{-1}[U(t+\varepsilon, x)-U(t, x)]=V_{\varepsilon}(t, x)
$$


exists and is continuous. Thus $U_{\varepsilon}$ satisfies the assumptions of the classical Itô formula. We apply it to $U_{\varepsilon}\left(t, X_{t}\right)$ and get an identity like (14) with $U_{\varepsilon}$ and $V_{\varepsilon}$ in place of $U$ and $V$. Given $t \geq 0$, the r.v. $U_{\varepsilon}\left(t, X_{t}(\omega)\right)=\varepsilon^{-1} \int_{t}^{t+\varepsilon} U\left(s, X_{t}(\omega)\right) d s$ converges $P$-a.s. to $U\left(t, X_{t}(\omega)\right)$ as $\varepsilon \rightarrow 0$ (we may also use the fact that, from equation (13), $U$ is globally bounded and continuous in $(t, x)$ on $\left.[0,+\infty) \times \mathbb{R}^{d}\right)$. Now we use the fact that $\left|V_{\varepsilon}\right|,\left|U_{\varepsilon}\right|,\left\|D U_{\varepsilon}\right\|,\left\|D^{2} U_{\varepsilon}\right\|$ are uniformly bounded in $(t, x, \varepsilon)$. The $P$-a.s. convergence of the Lebesgue integral is easy by dominated convergence theorem, since $\left|V_{\varepsilon}\right|$ is uniformly bounded in $(t, x, \varepsilon),\left|b_{s} \cdot D U_{\varepsilon}\right|$ is uniformly bounded in $(x, \varepsilon)$ by a constant times $\left|b_{s}\right|$ and similarly for $\left|\operatorname{Tr}\left(\sigma \sigma^{T} D^{2} U_{\varepsilon}\right)\right|$. Finally, the r.v. $\int_{0}^{t}\left\langle D U_{\varepsilon}\left(s, X_{s}\right), \sigma_{s} d W_{s}\right\rangle$ converges in probability to $\int_{0}^{t}\left\langle D U\left(s, X_{s}\right), \sigma_{s} d W_{s}\right\rangle$ because $\int_{0}^{t}\left\|\sigma_{s}^{T}\left(D U_{\varepsilon}\left(s, X_{s}\right)-D U\left(s, X_{s}\right)\right)\right\|^{2} d s$ converges in probability to zero (since it converges to zero $P$-a.s., again because the integrand is bounded by a constant times $\left.\left\|\sigma_{s} \sigma_{s}^{T}\right\|\right)$. The proof is complete.

We also need the following simple lemma.

Lemma 4 Under the assumptions of Theorem 2, let $u_{\lambda}$ be the solution to (9). Then

$$
\left\|D u_{\lambda}\right\|_{0} \rightarrow 0, \text { as } \lambda \rightarrow+\infty
$$

where the supremum is taken on $[0, \infty) \times \mathbb{R}^{d}$. The choice of $\lambda$ to have, for instance, $\left\|D u_{\lambda}\right\|_{0} \leq$ $1 / 2$, depends only on $\|b\|_{0}$ and $\|f\|_{0}$.

Proof. We write $\partial_{t} u_{\lambda}+\frac{1}{2} \Delta u_{\lambda}-\lambda u_{\lambda}=f-b \cdot D u_{\lambda}$. Using the well known estimate for the heat semigroup $\sup _{x \in \mathbb{R}^{d}}\left|D P_{t} g(x)\right| \leq C t^{-1 / 2} \sup _{x \in \mathbb{R}^{d}}|g(x)|, g \in C_{b}\left(\mathbb{R}^{d}\right), t>0$, and, differentiating in formula (12), we get easily, for any $\lambda>0$,

$$
\left\|D u_{\lambda}\right\|_{0} \leq \frac{c}{\lambda^{\frac{1}{2}}}\left(\|f\|_{0}+\left\|b \cdot D u_{\lambda}\right\|_{0}\right) \leq \frac{c}{\lambda^{\frac{1}{2}}}\|f\|_{0}+\frac{c}{\lambda^{\frac{1}{2}}}\|b\|_{0}\left\|D u_{\lambda}\right\|_{0} .
$$

Considering $\lambda>c^{2}\|b\|_{0}^{2}$, we get

$$
\left(1-\frac{c}{\lambda^{\frac{1}{2}}}\|b\|_{0}\right)\left\|D u_{\lambda}\right\|_{0} \leq \frac{c}{\lambda^{\frac{1}{2}}}\|f\|_{0}
$$

and the assertion follows. The proof is complete.

Theorem 5 Assume $b \in L^{\infty}\left(0, T ; C_{b}^{\alpha}\left(\mathbb{R}^{d} ; \mathbb{R}^{d}\right)\right)$. Then we have the following facts:

(i) (pathwise uniqueness) For every $s \in[0, T], x \in \mathbb{R}^{d}$, the stochastic equation (7) has a unique continuous adapted solution $X^{s, x}=\left(X_{t}^{s, x}(\omega), t \in[s, T], \omega \in \Omega\right)$.

(ii) (differentiable flow) There exists a stochastic flow $\phi_{s, t}$ of diffeomorphisms for equation (7). The flow is also of class $C^{1+\alpha^{\prime}}$ for any $\alpha^{\prime}<\alpha$.

(iii) (stability) Let $\left(b^{n}\right) \subset L^{\infty}\left(0, T ; C_{b}^{\alpha}\left(\mathbb{R}^{d} ; \mathbb{R}^{d}\right)\right)$ be a sequence of vector fields and $\phi^{n}$ be the corresponding stochastic flows. If $b^{n} \rightarrow b$ in $L^{\infty}\left(0, T ; C_{b}^{\alpha^{\prime}}\left(\mathbb{R}^{d} ; \mathbb{R}^{d}\right)\right)$ for some $\alpha^{\prime}>0$, then, for any $p \geq 1$,

$$
\begin{aligned}
\lim _{n \rightarrow \infty} \sup _{x \in \mathbb{R}^{d}} \sup _{0 \leq s \leq T} E\left[\sup _{r \in[s, T]}\left|\phi_{s, r}^{n}(x)-\phi_{s, r}(x)\right|^{p}\right]=0 \\
\quad \sup _{n \in \mathbb{N}} \sup _{x \in \mathbb{R}^{d}} \sup _{0 \leq s \leq T} E\left[\sup _{u \in[s, T]}\left\|D \phi_{s, u}^{n}(x)\right\|^{p}\right]<\infty, \\
\lim _{n \rightarrow \infty} \sup _{x \in \mathbb{R}^{d}} \sup _{0 \leq s \leq T} E\left[\sup _{r \in[s, T]}\left\|D \phi_{s, r}^{n}(x)-D \phi_{s, r}(x)\right\|^{p}\right]=0 .
\end{aligned}
$$


Proof. Step 1. (auxiliary parabolic systems). For $\lambda>0$ consider the (vector valued) solution $\psi \in L^{\infty}\left(0, \infty ; C_{b}^{2+\alpha}\left(\mathbb{R}^{d} ; \mathbb{R}^{d}\right)\right)$ to the parabolic system

$$
\partial_{t} \psi_{\lambda}+L^{b} \psi_{\lambda}-\lambda \psi_{\lambda}=-b, \quad(t, x) \in(0, \infty) \times \mathbb{R}^{d}
$$

provided by Theorem 2 with $f=-b$. Define

$$
\Psi_{\lambda}(t, x)=x+\psi_{\lambda}(t, x)
$$

Lemma 6 For $\lambda$ large enough, such that $\sup _{t \geq 0}\left\|D \psi_{\lambda}(t, \cdot)\right\|_{0}<1$ (see Lemma 4), the following statements hold:

(i) Uniformly in $t \in[0,+\infty), \Psi_{\lambda}$ has bounded first and second spatial derivatives and moreover the second (Fréchet) derivative $D_{x}^{2} \Psi_{\lambda}$ is globally $\alpha$-Hölder continuous.

(ii) for any $t \geq 0, \Psi_{\lambda}: \mathbb{R}^{d} \rightarrow \mathbb{R}^{d}$ is a non-singular diffeomorphism of class $C^{2}$.

(iii) $\Psi_{\lambda}^{-1}$ has bounded first and second spatial derivatives, uniformly in $t \in[0,+\infty)$.

Proof. Assertion (i) follows by Theorem 2 ,

(ii) Recall the classical Hadamard theorem (see for instance [32, page 330]): Let $g: \mathbb{R}^{d} \rightarrow \mathbb{R}^{d}$ be of class $C^{2}$. Suppose that $\lim _{|x| \rightarrow \infty}|g(x)|=+\infty$ and that the Jacobian matrix $D g(x)$ is an isomorphism of $\mathbb{R}^{d}$ for all $x \in \mathbb{R}^{d}$. Then $g$ is a $C^{2}$-diffeomorphism of $\mathbb{R}^{d}$. Applying this result to $\Psi_{\lambda}$, we get the assertion.

(iii) We know that $\Psi_{\lambda}^{-1}$ is of class $C^{2}$. Moreover

$$
\begin{aligned}
D \Psi_{\lambda}^{-1}(t, y) & =\left[D \Psi_{\lambda}\left(t, \Psi_{\lambda}^{-1} y\right)\right]^{-1}=\left[I+D \psi_{\lambda}\left(t, \Psi_{\lambda}^{-1} y\right)\right]^{-1} \\
& =\sum_{k \geq 0}\left(-D \psi_{\lambda}\left(t, \Psi_{\lambda}^{-1} y\right)\right)^{k}, \quad y \in \mathbb{R}^{d} .
\end{aligned}
$$

It follows that $\sup _{t \geq 0}\left\|D \Psi_{\lambda}^{-1}(t, \cdot)\right\|_{0} \leq \sum_{k \geq 0}\left(\sup _{t \geq 0}\left\|D \psi_{\lambda}(t, \cdot)\right\|_{0}\right)^{k}<\infty$. This shows the boundedness of the first derivative. Arguing in a similar way we get also the boundedness of the second derivative since

$$
D^{2} \Psi_{\lambda}^{-1}(t, y)=-\left[D \Psi_{\lambda}\left(t, \Psi_{\lambda}^{-1} y\right)\right]^{-1} D^{2} \Psi_{\lambda}\left(t, \Psi_{\lambda}^{-1}(t, y)\right)\left\{\left[D \Psi_{\lambda}\left(t, \Psi_{\lambda}^{-1} y\right)\right]^{-1}\right\}^{\otimes 2} .
$$

In the sequel we will use a value of $\lambda$ for which Lemma 6 holds and simply write $\psi$ and $\Psi$ for $\psi_{\lambda}$ and $\Psi_{\lambda}$.

Step 2. (conjugated SDE). Define

$$
\widetilde{b}(t, y)=-\lambda \psi\left(t, \Psi^{-1}(t, y)\right), \quad \widetilde{\sigma}(t, y)=D \Psi\left(t, \Psi^{-1}(t, y)\right)
$$

and consider, for every $s \in[0, T]$ and $y \in \mathbb{R}^{d}$, the SDE

$$
Y_{t}=y+\int_{s}^{t} \tilde{\sigma}\left(u, Y_{u}\right) d W_{u}+\int_{s}^{t} \widetilde{b}\left(u, Y_{u}\right) d u, \quad t \in[s, T] .
$$


This equation is equivalent to equation (7), in the following sense. If $X_{t}$ is a solution to (7), then $Y_{t}=\Psi\left(t, X_{t}\right)$ verifies equation (19) with $y=\Psi(s, x)$ : it is sufficient to apply Itô formula of Lemma 3 to $\Psi\left(t, X_{t}\right)$ and use equation (18). It is also possible to show that given a solution $Y_{t}$ of equation (19), then $X_{t}=\Psi^{-1}\left(t, Y_{t}\right)$ is a solution of (7) with $x=\Psi^{-1}(s, y)$, but we shall not use this fact.

Step 3. (proof of (i) and (ii)). Assertion (i) is known, see [36], but we give a proof based on our approach. We have clearly $\widetilde{b} \in L^{\infty}\left(0, T ; C_{b}^{1+\alpha}\left(\mathbb{R}^{d} ; \mathbb{R}^{d}\right)\right)$ and $\widetilde{\sigma} \in L^{\infty}\left(0, T ; C_{b}^{1+\alpha}\left(\mathbb{R}^{d} ; \mathbb{R}^{d \times d}\right)\right)$. By classical results (see [23, Ch. 2]) this implies existence and uniqueness of a strong solution $Y$ of equation (19) and even the existence of a $C^{1, \alpha^{\prime}}\left(\alpha^{\prime}<\alpha\right)$ stochastic flow of diffeomorphisms $\varphi_{s, t}$ associated to equation (19). Continuity in time is assumed in [23], but it can be easily extended to $L^{\infty}$ time dependence, as it is done in 25 even in greater generality. The uniqueness of $Y$ implies the path-wise uniqueness of solutions of the original SDE (7) since two solutions $X, \tilde{X}$ give rise to two processes $Y_{t}=\Psi\left(t, X_{t}\right)$ and $\tilde{Y}_{t}=\Psi\left(t, \tilde{X}_{t}\right)$ solving (19), then $Y=\tilde{Y}$ and then necessarily $X=\tilde{X}$. By the Yamada-Watanabe theorem path-wise uniqueness together with weak existence (which is a direct consequence of the Girsanov formula) gives the existence of the (unique) solution $\left(X_{t}^{x}\right)_{t \geq s}$ of eq. (7) starting from $x$ at time $s$. Moreover setting $\phi_{s, t}=\Psi_{t}^{-1} \circ \varphi_{s, t} \circ \Psi_{s}$ we realize that $\phi_{s, t}$ is the flow of (7) (in the sense that $X_{t}^{x}=\phi_{s, t}(x)$ ).

Step 4. (proof of (iii)). Let $\psi^{n}$ be the solution in $L^{\infty}\left(0, T ; C_{b}^{2+\alpha}\left(\mathbb{R}^{d} ; \mathbb{R}^{d}\right)\right.$ ) of the parabolic problem (18) associated to $b_{n}$. Notice that we can make a choice of $\lambda$ independent of $n$. Since $b^{n} \rightarrow b$ in $L^{\infty}\left(0, T ; C_{b}^{\alpha}\left(\mathbb{R}^{d} ; \mathbb{R}^{d}\right)\right)$, by Theorem 2 we have $\psi^{n} \rightarrow \psi$ in $L^{\infty}\left(0, T ; C_{b}^{2+\alpha}\left(\mathbb{R}^{d} ; \mathbb{R}^{d}\right)\right)$. To prove this last fact one has to write

$$
\partial_{t}\left(\psi^{n}-\psi\right)+L^{b}\left(\psi^{n}-\psi\right)-\lambda\left(\psi^{n}-\psi\right)=-\left(b^{n}-b\right)+\left(b^{n}-b\right) \cdot D \psi^{n}
$$

and use the bound

$$
\left\|\left(b^{n}-b\right) \cdot\left(D \psi^{n}-I\right)\right\|_{C_{b}^{\alpha}\left(\mathbb{R}^{d} ; \mathbb{R}^{d}\right)} \leq C\left\|b^{n}-b\right\|_{C_{b}^{\alpha}\left(\mathbb{R}^{d} ; \mathbb{R}^{d}\right)}
$$

which is true since, by Theorem 2 , $D \psi^{n}$ is uniformly bounded.

Consider the flows $\varphi_{s, t}^{n}=\Psi_{t}^{n} \circ \phi_{s, t}^{n} \circ\left(\Psi_{s}^{n}\right)^{-1}$ which satisfy

$$
\varphi_{s, t}^{n}(y)=y+\int_{s}^{t} \widetilde{b}^{n}\left(u, \varphi_{s, u}^{n}(y)\right) d u+\int_{s}^{t} \widetilde{\sigma}^{n}\left(u, \varphi_{s, u}^{n}(y)\right) \cdot d W_{u}
$$

We have $\widetilde{\sigma}^{n} \rightarrow \widetilde{\sigma}$ and $\widetilde{b}^{n} \rightarrow \widetilde{b}$ in $L^{\infty}\left(0, T ; C_{b}^{1+\alpha}\left(\mathbb{R}^{d} ; \mathbb{R}^{d \times d}\right)\right)$ and $L^{\infty}\left(0, T ; C_{b}^{1+\alpha}\left(\mathbb{R}^{d} ; \mathbb{R}^{d}\right)\right)$, respectively. By standard argument using the Gronwall lemma, the Doob inequality and the Burkholder inequality (compare, for instance, with the proof of [23, Theorem II.2.1]) we obtain the analog of (15) for the auxiliary flows $\varphi_{s, t}^{n}$ and $\varphi_{s, t}$. The estimates are standard so we leave them to the reader. We note only that we need to control the difference $\varphi_{s, t}^{n}\left(y_{n}\right)-\varphi_{s, t}(y)$ where $y_{n}=\Psi_{s}^{n}(x)$ and $y=\Psi_{s}(x)$. But $\left|\Psi_{s}^{n}(x)-\Psi_{s}(x)\right|$ is uniformly small in $x \in \mathbb{R}^{d}$ so we need to estimate $\varphi_{s, t}^{n}(y+v)-\varphi_{s, t}(y)$, uniformly in $y$, only with respect to a uniformly small variation $v$. Then it is not difficult to see that $E\left[\sup _{t \in[s, T]}\left|\varphi_{s, t}^{n}(y+v)-\varphi_{s, t}(y)\right|^{p}\right]$ is small in $y$ uniformly for large $n$ and small $v$. Finally, one has to check that $\left(\Psi_{s}^{n}\right)^{-1}$ converges to $\Psi_{s}^{-1}$ uniformly. This is due to the fact that $\Psi_{s}^{n}$ converges uniformly to $\Psi_{s}$ with its derivatives and the Jacobian $J \Psi_{s}$ is uniformly away from zero.

Concerning the derivative of the stochastic flow, first one can prove an inequality for $D \varphi_{s, t}^{n}(y)$ similar to (16) using the fact that the equation for $D \varphi_{s, t}^{n}(y)$ has the identity as initial condition 
and the coefficients $D \widetilde{b}^{n}\left(\phi_{s, u}^{n}\right)$ and $D \widetilde{\sigma}^{n}\left(\phi_{s, u}^{n}\right)$ are uniformly bounded functions (in all variables and $n$ ). Then one has to use the uniform boundedness of the derivatives of $\Psi_{s}^{n}$ and its inverse, to estimate $D \phi_{s, u}^{n}$.

Arguing as in the proof of [23, Theorem II.3.1], we get the following linear equation for the derivative $D \phi_{s, t}(x)$

$$
\begin{aligned}
{\left[D \Psi_{t}\left(\phi_{s, t}(x)\right)\right] D \phi_{s, t}(x) } & =D \Psi_{s}(x)+\int_{s}^{t}\left[D^{2} \Psi_{u}\left(\phi_{s, u}(x)\right)\right] D \phi_{s, u}(x) d W_{u} \\
& -\lambda \int_{s}^{t}\left[D \psi_{u}\left(\phi_{s, u}(x)\right)\right] D \phi_{s, u}(x) d u,
\end{aligned}
$$

$0 \leq s \leq t \leq T, x \in \mathbb{R}^{d}$. From the convergence $\psi^{n} \rightarrow \psi$ in $L^{\infty}\left(0, T ; C_{b}^{2+\alpha}\left(\mathbb{R}^{d} ; \mathbb{R}^{d}\right)\right)$ together with (16) and (21), we finally obtain, for any $p \geq 1$,

$$
\lim _{n \rightarrow \infty} \sup _{x \in \mathbb{R}^{d}} \sup _{0 \leq s \leq T} E\left[\sup _{u \in[s, T]}\left\|D \phi_{s, u}^{n}(x)-D \phi_{s, u}(x)\right\|^{p}\right]=0 .
$$

We show now that the inverse flow $\phi_{s, t}^{-1}$ is directly related to the solutions of a simple backward stochastic differential equations, of the same form as the original one (only the drift has opposite sign).

Lemma 7 Under Hypothesis 1 the process $\left(\phi_{s, t}^{-1}(y)\right)_{s \in[0, t]}$ is the unique solution of the backward $S D E$

$$
\phi_{s, t}^{-1}(y)=y-\int_{s}^{t} b\left(r, \phi_{r, t}^{-1}(y)\right) d r-\left[W_{t}-W_{s}\right]
$$

and

$$
\sup _{x \in \mathbb{R}^{d}} \sup _{0 \leq u \leq T} E\left[\sup _{s \in[0, u]}\left\|D \phi_{s, u}^{-1}(x)\right\|^{p}\right]<\infty
$$

for any $p \geq 1$.

Proof. We have $\phi_{s, t}(x)=x+\int_{s}^{t} b\left(r, \phi_{s, r}(x)\right) d r+W_{t}-W_{s}$ and then

$$
\phi_{s, t}\left(\phi_{s, t}^{-1}(y)\right)=\phi_{s, t}^{-1}(y)+\int_{s}^{t} b\left(r, \phi_{s, r}\left(\phi_{s, t}^{-1}(y)\right)\right) d r+W_{t}-W_{s}
$$

But $\phi_{s, r} \circ \phi_{s, t}^{-1}=\phi_{r, t}^{-1}$ and thus $y=\phi_{s, t}^{-1}(y)+\int_{s}^{t} b\left(r, \phi_{r, t}^{-1}(y)\right) d r+W_{t}-W_{s}$. The proof of the bound (23) is then similar to that of eq. (16) once taken into account the backward character of the equation.

Remark 8 We shall use the following consequence of the stability estimates in Th. 5: for any $r>0$ and $p \geq 1$,

$$
\begin{gathered}
\lim _{n \rightarrow \infty} E\left[\int_{B(r)} \sup _{t \in[0, T]}\left|\phi_{t}^{n}(y)-\phi_{t}(y)\right|^{p} d y\right]=0 \\
\lim _{n \rightarrow \infty} E\left[\int_{B(r)} \sup _{t \in[0, T]}\left\|D \phi_{t}^{n}(y)-D \phi_{t}(y)\right\|^{p} d y\right]=0 .
\end{gathered}
$$


Possibly passing to a subsequence, still denoted by $\phi^{n}$, this implies that P-a.s. the following property holds: for every $t \in[0, T], \phi_{t}^{n}$ and $D \phi_{t}^{n}$ converge to $\phi_{t}$ and $D \phi_{t}$ in $L^{p}\left(B(r) ; \mathbb{R}^{d}\right)$ and $L^{p}\left(B(r) ; \mathbb{R}^{d \times d}\right)$ respectively.

We finish the section with a result of independent interest. It concerns a special situation when the given vector field $b$ is of zero distributional divergence and gives rise to a measurepreserving flow.

Lemma 9 Assume $\operatorname{div} b(t, \cdot)=0, t \in[0, T]$, in the sense of distributions. Then the stochastic flow $\phi$ is $P$-a.s. measure-preserving, i.e., $J \phi_{s, t}(x)=1$, for all $0 \leq s \leq t \leq T$ and all $x \in \mathbb{R}^{d}$, P-a.s..

Proof. Let $b_{n}$ be $C_{b, T}^{\infty}$ vector fields that converge to $b$ in $C_{b, T}^{\alpha^{\prime}}$ for some $0<\alpha^{\prime}<\alpha$ and such that $\operatorname{div} b_{n}=0$. The functions $b_{n}$ can be constructed as in (6). Let $\phi^{n}$ be the associated smooth diffeomorphism. Applying [23, Theorem II.3.1] and the well known Liouville theorem, we get that the diffeomorphism $\phi^{n}$ preserves the Lebsegue measure since $b_{n}$ is a divergence-free vector field. Then $J \phi_{s, t}^{n}(x)=1$ for all $0 \leq s<t \leq T$ and all $x, P$-a.s.. Fix $x \in \mathbb{R}^{d}$ and $s \in[0, T]$. By (22), there exists a subsequence (possibly depending on $x, s$ and still denoted by $D \phi_{s, u}^{n}$ ) such that $P$-a.s.

$$
\sup _{s \leq u \leq T}\left\|D \phi_{s, u}^{n}(x)-D \phi_{s, u}(x)\right\|^{2} \rightarrow 0, \text { as } n \rightarrow \infty .
$$

We find that $J \phi_{s, t}(x)=1$, for any $x \in \mathbb{R}^{d}$, and so $\phi_{s, t}$ is a measure-preserving diffeomorphism $P$-a.s. for any $s<t \leq T$.

\section{Estimates on the derivative of the Jacobian}

The aim of this section is to prove Sobolev type estimates on the derivative of the Jacobian of the stochastic flow $\phi_{t}=\phi_{0, t}$ associated to equation (77) starting at 0 . These estimates will be crucial in the proof of uniqueness of weak solutions of the SPDE for $d \geq 2$ (the case $d=1$ will be treated differently).

The basic observation is that the (formal) expression

$$
\log J \phi_{t}(x)=\int_{0}^{t} \operatorname{div} b\left(s, \phi_{s}(x)\right) d s
$$

shows the opportunity of exploiting the Itô-Tanaka trick to regularize the integrated divergence of $b$. We make the following hypothesis on $\operatorname{div} b$ :

Hypothesis 2 There exists $p \in(2,+\infty)$, such that

$$
\operatorname{div} b \in L^{p}\left([0, T] \times \mathbb{R}^{d}\right)
$$

(where $\operatorname{div} b(t, \cdot)$ is understood in distributional sense).

To apply the Itô-Tanaka trick to eq. (24) the relevant PDEs results are classical $L^{p}$-parabolic estimates (see, for instance, [19]) which are based on the following function spaces. For $p \in$ 
$(1,+\infty)$, we consider the Banach space $H_{p}^{2}(T)$ of all functions $u \in L^{p}\left(0, T ; W^{2, p}\left(\mathbb{R}^{d}\right)\right)$ such that the distributional derivative $\partial_{t} u \in L^{p}\left([0, T] \times \mathbb{R}^{d}\right)$. The norm is given by

$$
\|u\|_{H_{p}^{2}(T)}\|u\|_{L^{p}\left(0, T ; W^{2, p}\left(\mathbb{R}^{d}\right)\right)}+\left\|\partial_{t} u\right\|_{L^{p}\left(0, T ; L^{p}\left(\mathbb{R}^{d}\right)\right)},
$$

$u \in H_{p}^{2}(T)$. The next result is well known (see, for instance, [19, Theorem 9 in Section 7.3]).

Theorem 10 Consider a Borel and bounded function $l:[0, T] \times \mathbb{R}^{d} \rightarrow \mathbb{R}^{d}$. For any function $f \in L^{p}\left([0, T] \times \mathbb{R}^{d}\right), p>1$, the Cauchy parabolic problem

$$
\left\{\begin{array}{r}
\frac{\partial F}{\partial t}+\frac{1}{2} \Delta F+l \cdot D F=f, \quad t \in[0, T[ \\
F(T, x)=0, \quad x \in \mathbb{R}^{d}
\end{array}\right.
$$

has a unique solution $F$ in the space $H_{p}^{2}(T)$ and $F \in C\left([0, T] ; W^{1, p}\left(\mathbb{R}^{d}\right)\right)$. Moreover, there exists a positive constant $C=C\left(p, d, T,\|l\|_{\infty}\right)$ such that

$$
\|F\|_{H_{p}^{2}(T)} \leq C\|f\|_{L^{p}\left([0, T] \times \mathbb{R}^{d}\right)} .
$$

If $p \geq 2$ the constant $C$ above can be chosen such that

$$
\sup _{t \in[0, T]}\|F(t, \cdot)\|_{W^{1, p}\left(\mathbb{R}^{d}\right)} \leq C\|f\|_{L^{p}\left([0, T] \times \mathbb{R}^{d}\right)} .
$$

We prove now a regularity result for the Jacobian $J \phi$.

Theorem 11 Under Hypotheses 1 and 2 we have $J \phi \in L^{2}\left(0, T ; W_{r}^{1,2}\right)$ P-a.s. for any $r>0$.

Proof. Step 1. Recall the chain rule for Sobolev function: if $f: \mathbb{R}^{d} \rightarrow \mathbb{R}$ is a continuous function, of class $W_{\text {loc }}^{1,2}\left(\mathbb{R}^{d}\right)$ and $g: \mathbb{R} \rightarrow \mathbb{R}$ is a $C^{\infty}$ function, then $g \circ f \in W_{\text {loc }}^{1,2}\left(\mathbb{R}^{d}\right)$ and

$$
\int_{B(r)}|D(g \circ f)(x)|^{2} d x \leq\left(\sup _{x \in B(r)}\left|g^{\prime}(f(x))\right|\right)^{2} \int_{B(r)}|D f(x)|^{2} d x
$$

for every $r>0$. Since $\log J \phi_{t}(x)$ is a continuous function, by the previous argument, if we prove that $\log J \phi_{t}(x) \in W_{l o c}^{1,2}\left(\mathbb{R}^{d}\right)$ for a.e. $(\omega, t) \in \Omega \times[0, T]$, then $J \phi_{t}(x) \in W_{l o c}^{1,2}\left(\mathbb{R}^{d}\right)$ for a.e. $(\omega, t) \in \Omega \times[0, T]$ and

$$
\int_{B(r)}\left|D J \phi_{t}(x)\right|^{2} d x \leq\left(\sup _{x \in B(r)}\left|J \phi_{t}(x)\right|\right)^{2} \int_{B(r)}\left|D \log J \phi_{t}(x)\right|^{2} d x .
$$

Integrating in $t \in[0, T]$ and recalling that $\sup _{x \in B(r), t \in[0, T]}\left|J \phi_{t}(x)\right|$ is finite $(P$-a.s. $)$ because $J \phi_{t}(x)$ is continuous in $(t, x)$, we see that in order to prove the theorem it is sufficient to prove that

$$
\log J \phi .(\cdot) \in L^{2}\left(0, T, W_{r}^{1,2}\right)
$$

for every $r>0$, for a.e. $\omega \in \Omega$. This will be proved by showing that

$$
\log J \phi .(\cdot) \in L^{2}\left(\Omega \times(0, T), W_{r}^{1,2}\right) .
$$


Step 2. Introduce $b^{\varepsilon}(t, x)=\left(\vartheta_{\varepsilon} * b(t, \cdot)\right)(x), \varepsilon>0$ and also set $b^{0}=b$. Let $\phi_{t}^{\varepsilon}$ be the flow corresponding to the SDE (17) with $b$ replaced by $b^{\varepsilon}$. By well known results (see [23]), we get, for any $\varepsilon>0$,

$$
\log J \phi_{t}^{\varepsilon}(x)=\int_{0}^{t} \operatorname{div} b^{\varepsilon}\left(s, \phi_{s}^{\varepsilon}(x)\right) d s .
$$

Since the noise is additive, this can be also proved in an elementary way by the $\omega$-wise application of the classical deterministic results to the equation

$$
\rho_{t}^{\varepsilon}(x)=x+\int_{0}^{t} g^{\varepsilon}\left(s, \rho_{s}^{\varepsilon}(x)\right) d s
$$

where $\rho_{t}^{\varepsilon}(x)=\phi_{t}^{\varepsilon}(x)-W_{t}, g^{\varepsilon}(t, y)=b^{\varepsilon}\left(t, y+W_{t}\right)\left(\right.$ one has $J \phi_{t}^{\varepsilon}(x)=J \rho_{t}^{\varepsilon}(x)$ and $\operatorname{div} g^{\varepsilon}(t, y)=$ $\left.\operatorname{div} b^{\varepsilon}\left(s, y+W_{t}\right)\right)$.

Note that, by Remark $8, J \phi_{t}^{\varepsilon}(x) \rightarrow J \phi_{t}(x)$ in $L^{2}\left(\Omega \times(0, T), L_{r}^{2}\right)$ as $\varepsilon \rightarrow 0^{+}$. Define

$$
\psi_{\varepsilon}(t, x)=\int_{0}^{t} \operatorname{div} b^{\varepsilon}\left(s, \phi_{s}^{\varepsilon}(x)\right) d s .
$$

Possibly passing to a sequence $\left(\varepsilon_{n}\right)_{n \geq 1}$, we have that

$$
\psi_{\varepsilon_{n}}(t, x) \rightarrow \log J \phi_{t}(x)
$$

a.e. in $t, x, \omega$, as $n \rightarrow \infty$. On the other hand, we have that $\left(\psi_{\varepsilon}\right)_{\varepsilon>0}$ is bounded in $L^{2}(\Omega \times$ $\left.(0, T) ; L_{r}^{2}\right)$. Indeed, we have, using that $p>2$ and (23),

$$
\begin{gathered}
\left(E \int_{0}^{T} \int_{B(r)}\left|\int_{0}^{t} \operatorname{div} b^{\varepsilon}\left(s, \phi_{s}^{\varepsilon}(x)\right) d s\right|^{2} d x d t\right)^{p / 2} \\
\leq C_{r, T} E \int_{0}^{T} \int_{B(r)}\left|\int_{0}^{t} \operatorname{div} b^{\varepsilon}\left(s, \phi_{s}^{\varepsilon}(x)\right) d s\right|^{p} d x d t \\
\leq C_{r, T}^{\prime} E \int_{0}^{T} d s \int_{B(r)}\left|\operatorname{div} b^{\varepsilon}\left(s, \phi_{s}^{\varepsilon}(x)\right)\right|^{p} d x \leq C_{r, T}^{\prime} \int_{0}^{T} d s \int_{\mathbb{R}^{d}}\left|\operatorname{div} b^{\varepsilon}(s, y)\right|^{p} E\left[J\left(\phi_{s}^{\varepsilon}\right)^{-1}(y)\right] d y \\
\leq C_{r, T}^{\prime \prime} \sup _{s \in[0, T], y \in \mathbb{R}^{d}} E\left[J\left(\phi_{s}^{\varepsilon}\right)^{-1}(y)\right] \int_{0}^{T} d s \int_{\mathbb{R}^{d}}\left|\operatorname{div} b^{\varepsilon}(s, y)\right|^{p} d y \leq C<\infty .
\end{gathered}
$$

where $C$ is independent on $\varepsilon>0$. Note that the previous computation also shows that $\psi_{\varepsilon}(t, x)$ is uniformly integrable on $\Omega \times[0, T] \times B(r)$.

By weak convergence we known that there exists a subsequence of $\left\{\psi_{\varepsilon_{n}}\right\}_{n \geq 1}$ (still denoted by $\left.\left\{\psi_{\varepsilon_{n}}\right\}_{n \geq 1}\right)$ which converges weakly in $L^{2}\left(\Omega \times(0, T) ; L_{r}^{2}\right)$ to some function $\psi$.

On the other hand, almost sure convergence and uniform integrability together imply that $\psi_{\varepsilon_{n}}$ converges strongly in $L^{1}(\Omega \times[0, T] \times B(r))$ to $\log J \phi$. It follows that, for any $\eta \in L^{\infty}(\Omega \times$ $[0, T] \times B(r))$, we have

$$
E \int_{0}^{T} \int_{B(r)} \psi(t, x) \eta(t, x) d t d x=E \int_{0}^{T} \int_{B(r)} \log J \phi_{t}(x) \eta(t, x) d t d x .
$$

giving $\psi=\log J \phi$ which means that $\psi_{\varepsilon_{n}}$ converges weakly in $L^{2}\left(\Omega \times(0, T) ; L_{r}^{2}\right)$ to $\log J \phi$. 
Step 3. To prove assertion (29) it is enough to check that the family $\left(\psi_{\varepsilon}\right)_{\varepsilon>0}$ is bounded in $L^{2}\left(\Omega \times(0, T) ; W_{r}^{1,2}\right)$. Indeed, once we have proved this fact, we can extract from the previous sequence $\psi_{\varepsilon_{n}}$ a subsequence which converges weakly in $L^{2}\left(\Omega \times(0, T) ; W_{r}^{1,2}\right)$ to some $\gamma$. This in particular implies that such subsequence converges weakly in $L^{2}\left(\Omega \times(0, T), L_{r}^{2}\right)$ to $\gamma$. By the previous step, we must have that $\gamma=J \phi$.

We introduce the following Cauchy problem, for $\varepsilon \geq 0$,

$$
\left\{\begin{array}{rr}
\frac{\partial F^{\varepsilon}}{\partial t}+\frac{1}{2} \Delta F^{\varepsilon}+D F^{\varepsilon} \cdot b^{\varepsilon}=\operatorname{div} b^{\varepsilon}, & t \in[0, T[ \\
F^{\varepsilon}(T, x)=0, & x \in \mathbb{R}^{d} .
\end{array}\right.
$$

Note that by Theorem 10 and since $p>2$ we have

$$
\left\|F^{\varepsilon}\right\|_{H_{p}^{2}(T)}+\sup _{t \in[0, T]}\left\|F^{\varepsilon}(t, \cdot)\right\|_{W^{1, p}\left(\mathbb{R}^{d}\right)} \leq C\|\operatorname{div} b\|_{L^{p}\left(0, T ; L^{p}\left(\mathbb{R}^{d}\right)\right)},
$$

for any $\varepsilon \geq 0$. Using Itô formula we find (remark that $F^{\varepsilon}(t, \cdot) \in C_{b}^{2}\left(\mathbb{R}^{d}\right)$ )

$$
F^{\varepsilon}\left(t, \phi_{t}^{\varepsilon}(x)\right)-F^{\varepsilon}(0, x)-\int_{0}^{t} D F^{\varepsilon}\left(s, \phi_{s}^{\varepsilon}(x)\right) \cdot d W_{s} \int_{0}^{t} \operatorname{div} b^{\varepsilon}\left(s, \phi_{s}^{\varepsilon}(x)\right) d s=\psi_{\varepsilon}(t, x) .
$$

By classical results (see [23]) the maps $\psi_{\varepsilon}(t, \cdot)$ are differentiable, $P$-a.s. and

$$
\begin{gathered}
D \psi_{\varepsilon}(t, x) D F^{\varepsilon}\left(t, \phi_{t}^{\varepsilon}(x)\right) D \phi_{t}^{\varepsilon}(x)-D F^{\varepsilon}(0, x) \\
-\int_{0}^{t} D^{2} F^{\varepsilon}\left(s, \phi_{s}^{\varepsilon}(x)\right) D \phi_{s}^{\varepsilon}(x) d W_{s}
\end{gathered}
$$

Since we already know that $\left(\psi_{\varepsilon}\right)_{\varepsilon>0}$ is bounded in $L^{2}\left(\Omega \times(0, T), L_{r}^{2}\right)$, to verify that $\left(\psi_{\varepsilon}\right)_{\varepsilon>0}$ is bounded in $L^{2}\left(\Omega \times(0, T) ; W_{r}^{1,2}\right)$, it is enough to prove that $\left(D \psi_{\varepsilon}\right)_{\varepsilon>0}$ is bounded in $L^{2}(\Omega \times$ $\left.(0, T), L_{r}^{2}\right)$.

To this purpose we only prove the bound for the critical term $\int_{0}^{t} D^{2} F^{\varepsilon}\left(s, \phi_{s}^{\varepsilon}(x)\right) D \phi_{s}^{\varepsilon}(x) d W_{s}$ in (33). The other terms are easier to estimate, we remark only that for the term $\operatorname{DF}^{\varepsilon}(0, x)$ we use eq. (32).

We show that there exists a constant $C>0$ (depending on $r$ and $T$ ) such that

$$
E\left[\int_{0}^{T} \int_{B(r)}\left|\int_{0}^{t} D^{2} F^{\varepsilon}\left(s, \phi_{s}^{\varepsilon}(x)\right) D \phi_{s}^{\varepsilon}(x) d W_{s}\right|^{2} d x d t\right] \leq C
$$

for every $\varepsilon>0$. Note that

$$
\begin{aligned}
& \int_{0}^{T} \int_{B(r)} E\left[\left|\int_{0}^{t} D^{2} F^{\varepsilon}\left(s, \phi_{s}^{\varepsilon}(x)\right) D \phi_{s}^{\varepsilon}(x) d W_{s}\right|^{2}\right] d x d t \\
& \leq T \int_{B(r)} E\left[\int_{0}^{T}\left|D^{2} F^{\varepsilon}\left(s, \phi_{s}^{\varepsilon}(x)\right) D \phi_{s}^{\varepsilon}(x)\right|^{2} d s\right] d x .
\end{aligned}
$$

According to (16), we have

$$
\int_{B(r)}\left(\int_{0}^{T} E\left[\left|D \phi_{s}^{\varepsilon}(x)\right|^{r}\right] d s\right) d x \leq C<\infty,
$$


for every $r \geq 1$, with $C$ independent on $\varepsilon$; therefore by the Hölder inequality on $\Omega \times B(r) \times[0, T]$, it is sufficient to prove that there exists $C>0$ such that, for any $\varepsilon>0$,

$$
\int_{B(r)} E\left[\int_{0}^{T}\left|D^{2} F^{\varepsilon}\left(s, \phi_{s}^{\varepsilon}(x)\right)\right|^{p} d s\right] d x \leq C<\infty .
$$

Step 4. Let us show (35). We have

$$
\begin{gathered}
E\left[\int_{0}^{T} d s \int_{B(r)}\left|D^{2} F^{\varepsilon}\left(s, \phi_{s}^{\varepsilon}(x)\right)\right|^{p} d x\right] \\
\leq \int_{0}^{T} d s \int_{\mathbb{R}^{d}}\left|D^{2} F^{\varepsilon}(s, y)\right|^{p} E\left[J\left(\phi_{s}^{\varepsilon}\right)^{-1}(y)\right] d y \\
\leq \sup _{s \in[0, T], y \in \mathbb{R}^{d}} E\left[J\left(\phi_{s}^{\varepsilon}\right)^{-1}(y)\right] \int_{0}^{T} d s \int_{\mathbb{R}^{d}}\left|D^{2} F^{\varepsilon}(s, y)\right|^{p} d y \leq C<\infty,
\end{gathered}
$$

where, using (23) and (32), $C$ is independent on $\varepsilon>0$. This proves (35) and ends the proof.

\section{Stochastic transport equation. Existence of weak solutions}

To avoid doubts, let us clarify a convention of language we use in the sequel. An element $u \in L^{\infty}\left(\Omega \times[0, T] \times \mathbb{R}^{d}\right)$ is an equivalence class. Given $\theta \in L^{1}\left(\mathbb{R}^{d}\right)$, the notation $\int_{\mathbb{R}^{d}} \theta(x) u(t, x) d x$ stands for an element of $L^{\infty}(\Omega \times[0, T])$ (so again an equivalence class) defined by Fubini theorem. When we say that $\int_{\mathbb{R}^{d}} \theta(x) u(t, x) d x$ has a continuous modification we mean that there exists an element in the equivalence class that is a continuous stochastic process (a process with continuous paths, $P$-a.s.). We choose this language so that it is the same as in the case when $u$ is a measurable function $u: \Omega \times[0, T] \times \mathbb{R}^{d} \rightarrow \mathbb{R}$ instead of an equivalence class (moreover, this way, when the property is true for the equivalent class it is true for all its representatives).

We still use the name 'stochastic process' for the elements of $L^{\infty}\left(\Omega \times[0, T] \times \mathbb{R}^{d}\right)$.

In the following definition we perform a Stratonovich integration. This is well defined when the integrator is a continuous semimartingale adapted to the filtration of the Brownian motion, see [23].

Definition 12 Let $b \in L_{l o c}^{1}\left([0, T] \times \mathbb{R}^{d} ; \mathbb{R}^{d}\right)$, div $b \in L_{l o c}^{1}\left([0, T] \times \mathbb{R}^{d}\right)$ and $u_{0} \in L^{\infty}\left(\mathbb{R}^{d}\right)$. A weak $L^{\infty}$-solution of the Cauchy problem (1) is a stochastic process $u \in L^{\infty}\left(\Omega \times[0, T] \times \mathbb{R}^{d}\right)$ such that, for every test function $\theta \in C_{0}^{\infty}\left(\mathbb{R}^{d}\right)$, the process $\int_{\mathbb{R}^{d}} \theta(x) u(t, x) d x$ has a continuous modification which is an $\mathcal{F}$-semimartingale and

$$
\begin{aligned}
\int_{\mathbb{R}^{d}} u(t, x) \theta(x) d x & =\int_{\mathbb{R}^{d}} u_{0}(x) \theta(x) d x \\
& +\int_{0}^{t} d s \int_{\mathbb{R}^{d}} u(s, x)[b(s, x) \cdot D \theta(x)+\operatorname{div} b(s, x) \theta(x)] d x \\
& +\sum_{i=1}^{d} \int_{0}^{t}\left(\int_{\mathbb{R}^{d}} u(s, x) D_{i} \theta(x) d x\right) \circ d W_{s}^{i} .
\end{aligned}
$$


In the previous definition we have used Stratonovich integrals since they are the natural ones in this framework. However, as usual, one can reformulate the problem in Itô form and avoid the semimartingale assumption.

Lemma 13 A process $u \in L^{\infty}\left(\Omega \times[0, T] \times \mathbb{R}^{d}\right)$ is a weak $L^{\infty}$-solution of the Cauchy problem (1) if and only if, for every test function $\theta \in C_{0}^{\infty}\left(\mathbb{R}^{d}\right)$, the process $\int_{\mathbb{R}^{d}} \theta(x) u(t, x) d x$ has a continuous $\mathcal{F}$-adapted modification and

$$
\begin{aligned}
\int_{\mathbb{R}^{d}} u(t, x) \theta(x) d x & =\int_{\mathbb{R}^{d}} u_{0}(x) \theta(x) d x \\
& +\int_{0}^{t} d s \int_{\mathbb{R}^{d}} u(s, x)[b(s, x) \cdot D \theta(x)+\operatorname{div} b(s, x) \theta(x)] d x \\
+ & \sum_{i=1}^{d} \int_{0}^{t}\left(\int_{\mathbb{R}^{d}} u(s, x) D_{i} \theta(x) d x\right) d W_{s}^{i}+\frac{1}{2} \int_{0}^{t} d s \int_{\mathbb{R}^{d}} u(s, x) \Delta \theta(x) d x
\end{aligned}
$$

for a.e. $(\omega, t) \in \Omega \times[0, T]$.

Proof. The relation between Itô and Stratonovich integrals is (see [23])

$$
\begin{aligned}
\int_{0}^{t} & \left(\int_{\mathbb{R}^{d}} u(s, x) D_{i} \theta(x) d x\right) \circ d W_{s}^{i} \\
& =\int_{0}^{t}\left(\int_{\mathbb{R}^{d}} u(s, x) D_{i} \theta(x) d x\right) d W_{s}^{i}+\frac{1}{2}\left[\int_{\mathbb{R}^{d}} u(\cdot, x) D_{i} \theta(x) d x, W^{i}\right]_{t}
\end{aligned}
$$

where $[\cdot, \cdot]_{t}$ denotes the joint quadratic variation. Only the martingale part of $\int_{\mathbb{R}^{d}} u(\cdot, x) D_{i} \theta(x) d x$ counts in the joint quadratic variation. If we start from definition [12, the martingale part of $\int_{\mathbb{R}^{d}} u(\cdot, x) D_{i} \theta(x) d x$ is (taking $D_{i} \theta$ in place of $\theta$ in the equation)

$$
\sum_{j=1}^{d} \int_{0}^{t}\left(\int_{\mathbb{R}^{d}} u(s, x) D_{i j}^{2} \theta(x) d x\right) d W_{s}^{j} .
$$

The same is true if, conversely, we start from equation (36). The joint quadratic variation is therefore equal to (see [23])

$$
\int_{0}^{t}\left(\int_{\mathbb{R}^{d}} u(s, x) D_{i j}^{2} \theta(x) d x\right) \cdot 1 d s .
$$

Summing over $i$, we get the result. The other details of the equivalence statement are easy. The proof is complete.

Remark 14 The presence of the Laplacian in the Itô formulation should not suggest that our SPDE has of parabolic nature. As clarified in the classical literature on parabolic SPDEs, an $S P D E$ in Itô form is parabolic when a super-parabolicity condition holds. If the second order operator in the drift has the form $\sum_{i, j=1}^{d} a_{i j} D_{j} D_{i} u$ and the first order part of the multiplicative noise has the form $\sum_{i, j=1}^{d} \sigma_{i j} D_{j} u d W_{t}^{i}$, then the operator

$$
\sum_{i, j=1}^{d}\left(a_{i j}-\frac{1}{2}\left(\sigma^{T} \sigma\right)_{i j}\right) D_{j} D_{i} u
$$

must be strongly elliptic (see for instance [34], [7]). In our case this operator is equal to zero. 
We may now prove a very general existence result, similarly to the deterministic case. The proof is essentially the same of that for SPDEs with monotone operators, see [22, 31, 34].

Theorem 15 Let $b \in L_{\text {loc }}^{1}\left([0, T] \times \mathbb{R}^{d} ; \mathbb{R}^{d}\right)$, $\operatorname{div} b \in L_{\text {loc }}^{1}\left([0, T] \times \mathbb{R}^{d}\right)$ and $u_{0} \in L^{\infty}\left(\mathbb{R}^{d}\right)$. Then there exists a weak $L^{\infty}$-solution of the SPDE (11).

Proof. Let $\eta^{\varepsilon}$ be a $C^{\infty}\left(\mathbb{R} \times \mathbb{R}^{d}\right)$ mollifier; let $\zeta \in C_{0}^{\infty}\left(\mathbb{R}^{d}\right)$ be such that $\zeta(x)=1$ for $|x| \leq 1$ and $\zeta(x)=0$ for $|x| \geq 2$. Define $b^{\varepsilon}=\eta^{\varepsilon} *(\zeta(\varepsilon \cdot) b)$ (for $t \notin[0, T]$, we set $b_{t}=0$ ). Note that each $b^{\varepsilon}$ is globally Lipschitz. Let $\phi^{\varepsilon}$ be the associated flow and define $u_{t}^{\varepsilon}(x)=u_{0}\left(\left(\phi^{\varepsilon}\right)_{t}^{-1}(x)\right)$. It is known (it can be checked by direct computation, see [24]) that this is the unique classical solutions of the associated transport equation, that written in weak Itô form is equation (36) with $u^{\varepsilon}$ and $b^{\varepsilon}$ in place of $u$ and $b$. From the representation in terms of the flow we immediately have $\sup _{x, \omega, t}\left|u_{t}^{\varepsilon}(x)(\omega)\right| \leq C$ uniformly in $\varepsilon$ so there exists a sequence $u^{\varepsilon_{n}}$ converging weak-* in $L^{\infty}\left(\Omega \times[0, T] \times \mathbb{R}^{d}\right)$ and weakly in $L^{2}(\Omega \times[0, T] \times B(N))$ for every integer $N>0$, to some $u$, which belongs to these spaces. For shortness, let us denote: $\varepsilon_{n}$ by $\varepsilon$; for every $\theta \in L^{1}\left(\mathbb{R}^{d}\right)$, $\int_{\mathbb{R}^{d}} \theta(x) u^{\varepsilon}(t, x) d x$ by $u_{t}^{\varepsilon}(\theta)$, including $u_{t}(\theta)$ as the case $\varepsilon=0 ; b^{\varepsilon}(s, x)$ by $b_{s}^{\varepsilon}(x)$, again also for $\varepsilon=0$.

We follow here the arguments of [31], Chapter III. Let $\theta \in C_{0}^{\infty}\left(\mathbb{R}^{d}\right)$. The process $u^{\varepsilon}(\theta)$ is non anticipative (by its definition) and converges weakly in $L^{2}(\Omega \times[0, T]$ ) (by the weak convergence of $u^{\varepsilon}$ to $u$ in $L^{2}(\Omega \times[0, T] \times B(N))$ for $N$ such that $B(N)$ contains the support of $\left.\theta\right)$. The space of non anticipative processes is a closed subspace of $L^{2}(\Omega \times[0, T])$, hence weakly closed. Therefore $u(\theta)$ is non anticipative. Thus Itô integrals of $u(\theta)$ (which is also bounded) are well defined. Moreover, the mapping $h \mapsto \int_{0}^{\cdot} h \cdot d W$ is linear continuous from the space of nonanticipative $L^{2}\left(\Omega \times[0, T] ; \mathbb{R}^{d}\right)$-processes to $L^{2}(\Omega \times[0, T])$; then it is also weakly continuous. Therefore $\int_{0} u_{s}^{\varepsilon}(D \theta) \cdot d W_{s}$ converges weakly in $L^{2}(\Omega \times[0, T])$ to $\int_{0}^{\cdot} u_{s}(D \theta) \cdot d W_{s}$.

Moreover we have $b^{\varepsilon} \rightarrow b$ and $\operatorname{div} b^{\varepsilon} \rightarrow \operatorname{div} b$ in $L_{\text {loc }}^{1}\left([0, T] \times \mathbb{R}^{d}\right)$. For every $\varepsilon \geq 0$, define

$$
G_{t}^{\varepsilon}\left(u^{\varepsilon}, \theta\right)=u_{t}^{\varepsilon}(\theta)-u_{0}(\theta)-\int_{0}^{t} u_{s}^{\varepsilon}\left(b_{s}^{\varepsilon} \cdot D \theta+\theta \operatorname{div} b_{s}^{\varepsilon}\right) d s-\frac{1}{2} \int_{0}^{t} u_{s}^{\varepsilon}(\Delta \theta) d s .
$$

It is not difficult to check that $G^{\varepsilon}\left(u^{\varepsilon}, \theta\right)$ converges weakly to $G(u, \theta)$ in $L^{2}(\Omega \times[0, T])$. To this purpose notice that $b^{\varepsilon} \cdot D \theta+\theta \operatorname{div} b^{\varepsilon}$ converges strongly to $b \cdot D \theta+\theta \operatorname{div} b$ in $L^{1}\left([0, T] \times \mathbb{R}^{d}\right)$.

Therefore we may pass to the weak $L^{2}(\Omega \times[0, T])$-limit in the equation for $u^{\varepsilon}$ and prove that $u$ satisfies equation (36) for a.e. $(\omega, t) \in \Omega \times[0, T]$.

Finally, the right-hand-side of equation (36) defines a continuous stochastic process. Therefore $u(\theta)$ has a continuous modification. The proof is complete.

Under more restrictive conditions we may construct a solution related to the stochastic flow.

Theorem 16 Assume that hypothesis 1 holds and $\operatorname{div} b \in L_{\text {loc }}^{1}\left([0, T] \times \mathbb{R}^{d}\right)$. Given $u_{0} \in L^{\infty}\left(\mathbb{R}^{d}\right)$, the stochastic process $u(t, x)$ defined as $u(t, x)=u_{0}\left(\phi_{t}^{-1}(x)\right)$ is a solution of (1)).

Proof. Step 1. We first prove the claim when $u_{0}$ has support in some ball $B(R)$. Let $b^{\varepsilon}$ be a regularization of $b$ as described in Section 1, It converges to $b$ in $L^{\infty}\left(0, T ; C_{b}^{\alpha^{\prime}}\left(\mathbb{R}^{d} ; \mathbb{R}^{d}\right)\right)$ for every $\alpha^{\prime}<\alpha$. We apply the argument of the previous proof to this particular $b^{\varepsilon}$ and the associated solution $u_{t}^{\varepsilon}(x)=u_{0}\left(\left(\phi^{\varepsilon}\right)_{t}^{-1}(x)\right)$. Let $u$ be one of its weak (and weak-*) limits, as described by the previous proof, and $u^{\varepsilon_{n}}$ the corresponding sequence. We know that $u$ is a solution of (1). We shall use that $u^{\varepsilon_{n}}$ converges weakly to $u$ in $L^{2}(\Omega \times[0, T] \times B(N))$ for every 
integer $N>0$. Thus, for every $\theta \in C_{0}^{\infty}\left(\mathbb{R}^{d}\right), u^{\varepsilon_{n}}(\theta)$ (we use the notations of the previous proof) converges to $u(\theta)$ weakly in $L^{2}(\Omega \times[0, T])$.

Let us prove that a further subsequence, still denoted by $u_{t}^{\varepsilon_{n}}(\theta)$, converges to the quantity $\int_{\mathbb{R}^{d}} u_{0}\left(\phi_{t}^{-1}(x)\right) \theta(x) d x$ for a.e. $(\omega, t) \in \Omega \times[0, T]$. Since $u_{t}^{\varepsilon_{n}}(\theta)$ is equibounded, the convergence is also strong in $L^{2}(\Omega \times[0, T])$ and thus weak. Therefore $u_{t}(\theta)=\int_{\mathbb{R}^{d}} u_{0}\left(\phi_{t}^{-1}(x)\right) \theta(x) d x$. This implies the claim of the theorem. Let $t \in[0, T]$ be given. We have

$$
u_{t}^{\varepsilon_{n}}(\theta)=\int_{B(R)} u_{0}(y) \theta\left(\phi_{t}^{\varepsilon_{n}}(y)\right) J \phi_{t}^{\varepsilon_{n}}(y) d y
$$

By Remark 8, up to a subsequence still denoted by $\varepsilon_{n}, \theta\left(\phi_{t}^{\varepsilon_{n}}(\cdot)\right) J \phi_{t}^{\varepsilon_{n}}(\cdot)$ converges in $L^{1}(B(R))$ to $\theta\left(\phi_{t}(\cdot)\right) J \phi_{t}(\cdot), P$-a.s. and thus, $P$-a.s., $u_{t}^{\varepsilon_{n}}(\theta)$ converges to $\int_{B(R)} u_{0}(y) \theta\left(\phi_{t}(y)\right) J \phi_{t}(y) d y$. This is what we wanted to prove.

Step 2. Consider now a general $u_{0} \in L^{\infty}\left(\mathbb{R}^{d}\right)$. Let $\zeta \in C_{0}^{\infty}\left(\mathbb{R}^{d}\right)$ be such that $\zeta(x)=1$ for $|x| \leq 1, \zeta(x)=0$ for $|x| \geq 2, \zeta(x) \in[0,1]$ for all $x \in \mathbb{R}^{d}$. Define $u_{0}^{n}(x)=u_{0}(x) \zeta\left(n^{-1} x\right)$. Let $u^{n}$ be the corresponding solution given by step 1 . We have

$$
u^{n}(t, x)=u_{0}\left(\phi_{t}^{-1}(x)\right) \zeta\left(n^{-1} \phi_{t}^{-1}(x)\right) .
$$

The function $u^{n}$ converges pointwise to $u(t, x)=u_{0}\left(\phi_{t}^{-1}(x)\right)$ and, because of equiboundedness, strongly in $L^{2}(\Omega \times[0, T] \times B(N))$ for every integer $N>0$, and weak-* in $L^{\infty}\left(\Omega \times[0, T] \times \mathbb{R}^{d}\right)$. Moreover, $u^{n}$ verifies equation (36). It is now easy to repeat the proof of the previous theorem and check that $u$ is a solution. The proof is complete.

\section{$5 \quad$ Uniqueness of weak solutions}

In this section, we prove uniqueness for our SPDE. Our main results are Theorems 20 and 21 ,

Let us recall that in the deterministic case with non-regular vector fields $b$, uniqueness of weak $L^{\infty}$-solutions is proved by means of the concept of renormalized solutions, see [10, 2]. The technical tool is a commutator lemma, where the role of some of the assumptions on $b$ is immediately visible. Given $g \in L_{l o c}^{\infty}\left(\mathbb{R}^{d}\right), v \in L_{l o c}^{1}\left(\mathbb{R}^{d}, \mathbb{R}^{d}\right)$, div $v \in L_{l o c}^{1}\left(\mathbb{R}^{d}\right)$, denote by $v \cdot D g$ the distribution defined on smooth compactly supported test functions $\rho$ as

$$
(v \cdot D g)(\rho)=-\int g v \cdot D \rho d x-\int g \rho \operatorname{div} v d x
$$

Given mollifiers $\vartheta_{\varepsilon}$ as in Section 1, define the commutator $\mathcal{R}_{\varepsilon}[v, g]$ as the smooth function

$$
\mathcal{R}_{\varepsilon}[v, g](x)=\left[\vartheta_{\varepsilon} *(v \cdot D g)-v \cdot D\left(\vartheta_{\varepsilon} * g\right)\right](x) .
$$

In the case $d=1$ we shall use the following classical form of the commutator lemma.

Lemma 17 Given $v \in W_{l o c}^{1,1}\left(\mathbb{R}^{d}, \mathbb{R}^{d}\right), g \in L_{l o c}^{\infty}\left(\mathbb{R}^{d}\right)$, we have

$$
\int_{B(r)}\left|\mathcal{R}_{\varepsilon}[v, g](x)\right| d x \leq C\|v\|_{W_{r+2}^{1,1}}\|g\|_{L_{r+2}^{\infty}}
$$

for all $r>0$, for some constant $C>0$ independent of $\varepsilon, v, g$ and $r$. Moreover, $\lim _{\varepsilon \rightarrow 0}$ $\int_{B(r)}\left|\mathcal{R}_{\varepsilon}[v, g](x)\right| d x=0$. 
Proof. In a global form on $\mathbb{R}^{d}$ it is proved for instance in [29, Ch. 2], Lemma 2.3. The local form on balls $B(r)$ can be easily deduced from the global form by multiplying $v$ and $g$ by a smooth function which takes values in $[0,1]$, and it is equal to 1 on $B(r+1)$ and to 0 outside $B(r+2)$.

The strong $L^{1}$ convergence of commutators requires some weak form of differentiability of $v$ or $g$. We impose such differentiability conditions only in the case $d=1$ where $\operatorname{div} b=D b$, or in the case of $B V_{l o c}$ solutions treated in Appendix A. For $d>1$ and $L^{\infty}$ solutions, taking advantage of the presence of the flow, we can prove uniqueness by means the distributional convergence of commutators composed with the flow. This composition put into play the first derivatives of the Jacobian of the flow.

The special estimates of Section 3 becomes the main tool to prove our first uniqueness result (see Theorem 201).

We start by giving some preliminary easy estimates on the distributional commutator and on its composition with a $C^{1}$ diffeomorphism.

Lemma 18 Given $v \in L_{l o c}^{\infty}\left(\mathbb{R}^{d}, \mathbb{R}^{d}\right)$, div $v \in L_{\text {loc }}^{1}\left(\mathbb{R}^{d}\right), g \in L_{l o c}^{\infty}\left(\mathbb{R}^{d}\right) ;$ for any $\rho \in C_{r}^{\infty}\left(\mathbb{R}^{d}\right)$ and for sufficiently small $\varepsilon$ we have

$$
\left|\int \mathcal{R}_{\varepsilon}[v, g](x) \rho(x) d x\right| \leq 2\|g\|_{L_{r+1}^{\infty}}\left[\|\operatorname{div} v\|_{L_{r+1}^{1}}\|\rho\|_{L_{r}^{\infty}}+\|v\|_{L_{r+1}^{\infty}}\|D \rho\|_{L_{r}^{1}}\right]
$$

and

$$
\lim _{\varepsilon \rightarrow 0} \int \mathcal{R}_{\varepsilon}[v, g](x) \rho(x) d x=0 .
$$

Proof. The proof proceeds as the one of Lemma 17 from [29, Ch. 2]: we prove the inequality for regular fields, then one can extend to non-regular ones and prove the convergence again first in the regular case and then apply an approximation procedure which we will omit. Let us rewrite the expressions in a suitable way:

$$
\begin{aligned}
\int \mathcal{R}_{\varepsilon}[v, g](x) \rho(x) d x= & \int\left[\vartheta_{\varepsilon} *(v \cdot D g)\right]\left(x^{\prime}\right) \rho\left(x^{\prime}\right) d x^{\prime}-\int\left[v \cdot D\left(\vartheta_{\varepsilon} * g\right)\right](x) \rho(x) d x \\
= & \iint\left[-g\left(x^{\prime}\right) v\left(x^{\prime}\right) D_{x^{\prime}} \vartheta_{\varepsilon}\left(x-x^{\prime}\right)-g\left(x^{\prime}\right) \vartheta_{\varepsilon}\left(x-x^{\prime}\right) \operatorname{div} v\left(x^{\prime}\right)\right] \rho(x) d x d x^{\prime} \\
& +\iint\left[g\left(x^{\prime}\right) v(x) \vartheta_{\varepsilon}\left(x-x^{\prime}\right) D_{x} \rho(x)+g\left(x^{\prime}\right) \rho(x) \vartheta_{\varepsilon}\left(x-x^{\prime}\right) \operatorname{div} v(x)\right] d x d x^{\prime}
\end{aligned}
$$

Using $D_{x^{\prime}} \vartheta_{\varepsilon}\left(x-x^{\prime}\right)=-D_{x} \vartheta_{\varepsilon}\left(x-x^{\prime}\right)$ and integrating by parts in $x$ the first term we get

$$
\begin{aligned}
& =\iint g\left(x^{\prime}\right) \vartheta_{\varepsilon}\left(x-x^{\prime}\right) D_{x} \rho(x)\left[v(x)-v\left(x^{\prime}\right)\right] d x d x^{\prime} \\
& \quad+\iint g\left(x^{\prime}\right) \rho(x) \vartheta_{\varepsilon}\left(x-x^{\prime}\right)\left[\operatorname{div} v(x)-\operatorname{div} v\left(x^{\prime}\right)\right] d x d x^{\prime} .
\end{aligned}
$$

Assume $\varepsilon$ so small that the support of $\vartheta_{\varepsilon}$ has diameter less than one. We have (using standard estimates on convolutions)

$$
\left|\int \mathcal{R}_{\varepsilon}[v, g](x) \rho(x) d x\right| \leq 2\|g\|_{L_{r+1}^{\infty}}\|v\|_{L_{r+1}^{\infty}}\|D \rho\|_{L_{r}^{1}}+2\|\rho\|_{L_{r}^{\infty}}\|g\|_{L_{r+1}^{\infty}}\|\operatorname{div} v\|_{L_{r+1}^{1}} .
$$


Corollary 19 Let $\phi$ be an $C^{1}$ diffeomorphism of $\mathbb{R}^{d}$. Assume $v \in L_{\mathrm{loc}}^{\infty}\left(\mathbb{R}^{d}, \mathbb{R}^{d}\right)$, div $v \in$ $L_{\mathrm{loc}}^{1}\left(\mathbb{R}^{d}\right), g \in L_{\mathrm{loc}}^{\infty}\left(\mathbb{R}^{d}\right)$. Moreover, if $d>1$, assume also $J \phi^{-1} \in W_{\text {loc }}^{1,1}\left(\mathbb{R}^{d}\right)$. Then for any $\rho \in C_{0}^{\infty}\left(\mathbb{R}^{d}\right)$ there exists a constant $C_{\rho}>0$ such that, given any $R>0$ such that $\operatorname{supp}\left(\rho \circ \phi^{-1}\right) \subseteq B(R)$, we have:

- for $d>1$,

$$
\begin{aligned}
& \left|\int \mathcal{R}_{\varepsilon}[v, g](\phi(x)) \rho(x) d x\right| \\
& \quad \leq C_{\rho}\|g\|_{L_{R+1}^{\infty}}\left[\|\operatorname{div} v\|_{L_{R+1}^{1}}\left\|J \phi^{-1}\right\|_{L_{R}^{\infty}}+\|v\|_{L_{R+1}^{\infty}}\left(\left\|D \phi^{-1}\right\|_{L_{R}^{\infty}}+\left\|D J \phi^{-1}\right\|_{L_{R}^{1}}\right)\right]
\end{aligned}
$$

- for $d=1$,

$$
\left|\int \mathcal{R}_{\varepsilon}[v, g](\phi(x)) \rho(x) d x\right| \leq C_{\rho}\left\|J \phi^{-1}\right\|_{L_{R}^{\infty}}\|v\|_{W_{R+2}^{1,1}}\|g\|_{L_{R+2}^{\infty}} .
$$

In both cases we have

$$
\lim _{\varepsilon \rightarrow 0} \int \mathcal{R}_{\varepsilon}[v, g](\phi(x)) \rho(x) d x=0 .
$$

Proof. By a change of variables we have $\int \mathcal{R}_{\varepsilon}[v, g](\phi(x)) \rho(x) d x=\int \mathcal{R}_{\varepsilon}[v, g](y) \rho_{\phi}(y) d x$ where the function $\rho_{\phi}(y)=\rho\left(\phi^{-1}(y)\right) J \phi^{-1}(y)$ has the support strictly contained in the ball of radius $R$. For $d>1$, by the previous lemma

$$
\left|\int \mathcal{R}_{\varepsilon}[v, g](\phi(x)) \rho(x) d x\right| \leq 2\|g\|_{L_{R+1}^{\infty}}\left[\|\operatorname{div} v\|_{L_{R+1}^{1}}\left\|\rho_{\phi}\right\|_{L_{R}^{\infty}}+\|v\|_{L_{R+1}^{\infty}}\left\|D \rho_{\phi}\right\|_{L_{R}^{1}}\right] .
$$

To conclude, it is sufficient to note that $\left\|\rho_{\phi}\right\|_{L_{R}^{\infty}} \leq\|\rho\|_{L_{r}^{\infty}}\left\|J \phi^{-1}\right\|_{L_{R}^{\infty}}$ and, denoting by $\left[D \phi^{-1}(y)\right]^{*}$ the adjoint matrix of $\left[D \phi^{-1}(y)\right]$,

$$
\begin{aligned}
\left\|D \rho_{\phi}\right\|_{L_{R}^{1}} & \leq\left\|\left[D \phi^{-1}(\cdot)\right]^{*}\left(D \rho \circ \phi^{-1}\right)(\cdot) J \phi^{-1}(\cdot)\right\|_{L_{R}^{1}}+\left\|\left(\rho \circ \phi^{-1}\right) \cdot D J \phi^{-1}\right\|_{L_{R}^{1}} \\
& \leq\left\|D \phi^{-1}\right\|_{L_{R}^{\infty}}\|D \rho\|_{L_{R}^{1}}+\|\rho\|_{L_{R}^{\infty}}\left\|D J \phi^{-1}\right\|_{L_{R}^{1}} .
\end{aligned}
$$

Given the bound the convergence follows by approximation.

For $d=1$, we simply have

$$
\begin{aligned}
\left|\int \mathcal{R}_{\varepsilon}[v, g](\phi(x)) \rho(x) d x\right| & =\left|\int \mathcal{R}_{\varepsilon}[v, g](y) \rho_{\phi}(y) d x\right| \\
& \leq\left\|\rho_{\phi}\right\|_{L_{R}^{\infty}} \int_{B(R)}\left|\mathcal{R}_{\varepsilon}[v, g](y)\right| d x \\
& \leq C_{\rho}\left\|J \phi^{-1}\right\|_{L_{R}^{\infty}}\|v\|_{W_{R+2}^{1,1}}\|g\|_{L_{R+2}^{\infty}}
\end{aligned}
$$

where we have used Lemma 17, The proof is complete.

We are now ready to prove our first uniqueness result of weak $L^{\infty}$-solutions to the SPDE (1).

Theorem 20 Assume that Hypothesis 1 holds true. Moreover, assume Hypothesis 2 for any $d \geq 1$ or simply $D b \in L_{l o c}^{1}([0, T] \times \mathbb{R})$ in the case $d=1$. Then, for every $u_{0} \in L^{\infty}$, there exists a unique weak $L^{\infty}$-solution of the Cauchy problem (1) which has the form $u(t, x, \omega)=$ $u_{0}\left(\phi_{t}^{-1}(\omega) x\right)$. 
Proof. Step 1. By linearity we have to prove that a weak $L^{\infty}$-solution with initial condition $u_{0}=0$ vanishes identically. Let us denote by $u$ such a solution. For $y \in \mathbb{R}^{d}$ fixed, $\varepsilon>0$, let us choose the test function $\theta(x)=\vartheta_{\varepsilon}(y-x)$ in Definition 12, Let us define $u^{\varepsilon}(t, \cdot)=\vartheta_{\varepsilon} * u(t, \cdot)$. We get

$$
u^{\varepsilon}(t, y)=\int_{0}^{t} A_{\varepsilon}(s, y) d s+\sum_{i=1}^{d} \int_{0}^{t} B_{\varepsilon}^{(i)}(s, y) \circ d W_{s}^{i}
$$

where

$$
\begin{aligned}
A_{\varepsilon}(t, y) & =\int_{\mathbb{R}^{d}} u(t, x)\left\{b(t, x) \cdot D_{x}\left[\vartheta_{\varepsilon}(y-x)\right]+\operatorname{div} b(t, x) \vartheta_{\varepsilon}(y-x)\right\} d x \\
B_{\varepsilon}^{(i)}(t, y) & =\int_{\mathbb{R}^{d}} u(t, x) D_{i}\left[\vartheta_{\varepsilon}(y-x)\right] d x .
\end{aligned}
$$

All these functions of $y$, namely $u^{\varepsilon}(t, y), A_{\varepsilon}(t, y), B_{\varepsilon}^{(i)}(t, y)$, are bounded measurable in $t$, adapted, smooth (of class $C^{3}$ is required for the next computation) in $y$ (from (i) and (ii) of definition 12). As a minor remark, we know that $\int_{\mathbb{R}^{d}} u(t, x) \rho(x) d x$ is adapted when $\rho \in$ $C_{0}^{\infty}\left(\mathbb{R}^{d}\right)$, by definition of solution, and by approximation the same property holds for $\rho \in$ $L^{1}\left(\mathbb{R}^{d}\right)$. From the Stratonovich version of Kunita-Itô-Wentzel formula (see [23, Th. 8.3 page 188]), we have

$$
\begin{aligned}
d u^{\varepsilon}\left(t, \phi_{t}(x)\right) & =A_{\varepsilon}\left(t, \phi_{t}(x)\right) d t+\sum_{i=1}^{d} B_{\varepsilon}^{(i)}\left(t, \phi_{t}(x)\right) \circ d W_{t}^{i} \\
& +\left(b \cdot D u^{\varepsilon}\right)\left(t, \phi_{t}(x)\right) d t+\sum_{i=1}^{d}\left(D_{i} u^{\varepsilon}\right)\left(t, \phi_{t}(x)\right) \circ d W_{t}^{i}
\end{aligned}
$$

But

$$
\left(D_{i} u^{\varepsilon}\right)(t, y)=-\int_{\mathbb{R}^{d}} u(t, x) D_{i}\left[\vartheta_{\varepsilon}(y-x)\right] d x
$$

hence

$$
d u^{\varepsilon}\left(t, \phi_{t}(x)\right)=\left[A_{\varepsilon}\left(t, \phi_{t}(x)\right)+\left(b \cdot D u^{\varepsilon}\right)\left(t, \phi_{t}(x)\right)\right] d t
$$

namely (recall that the initial condition is zero)

$$
u^{\varepsilon}\left(t, \phi_{t}(x)\right)=-\int_{0}^{t} \mathcal{R}_{\varepsilon}\left[b_{s}, u_{s}\right]\left(\phi_{s}(x)\right) d s
$$

where $\mathcal{R}_{\varepsilon}\left[b_{s}, u_{s}\right]$ is the commutator defined above.

The Stratonovich version of Kunita-Itô-Wentzel formula as given in [23, Th. 8.3 page 188] is optimized only with respect to the martingale parts of the processes: the theorems are stated for integrals of the form $\int_{0}^{t} f_{s}(x) d M_{s}$ where $M$ is a continuous semimartingale, but the assumptions on $f$ are those necessary to deal with martingales, not simply with processes of bounded variations. For the bounded variation parts, which in our case take the form $\int_{0}^{t} f_{s}(x) d s$, much weaker assumptions are needed. This is in analogy with Lemma 3 proved above and applies in particular to the random function $U(t, y)$ given by the integral $\int_{0}^{t} A_{\varepsilon}(s, y) d s: A_{\varepsilon}$ does not satisfies the conditions of [23, Th. 8.3 page 188] but $U(t, y)$ satisfies those of Lemma 3 . The Stratonovich version of Kunita-Itô-Wentzel formula extends to this case. 
Step 2. For every $\rho \in C_{0}^{\infty}\left(\mathbb{R}^{d}\right)$ (see the definition above) we have

$$
\int u^{\varepsilon}\left(t, \phi_{t}(x)\right) \rho(x) d x=\int u^{\varepsilon}(t, y) \rho\left(\phi_{t}^{-1}(y)\right) J \phi_{t}^{-1}(y) d y .
$$

Given $t \in[0, T]$, with probability one, $u^{\varepsilon}(t, \cdot)$ converges weak-* to $u(t, \cdot)$ as $\varepsilon \rightarrow 0$. Moreover, $P$-a.s., the function $y \mapsto \rho\left(\phi_{t}^{-1}(y)\right) J \phi_{t}^{-1}(y)$ is integrable, since it is continuous and with compact support. Hence, $P$-a.s.,

$$
\begin{aligned}
\lim _{\varepsilon \rightarrow 0} \int u^{\varepsilon}\left(t, \phi_{t}(x)\right) \rho(x) d x & =\int u(t, y) \rho\left(\phi_{t}^{-1}(y)\right) J \phi_{t}^{-1}(y) d y \\
& =\int u\left(t, \phi_{t}(x)\right) \rho(x) d x
\end{aligned}
$$

Therefore we have $P$-a.s.

$$
\int u\left(t, \phi_{t}(x)\right) \rho(x) d x=\lim _{\varepsilon \rightarrow 0} \int\left(\int_{0}^{t} \mathcal{R}_{\varepsilon}\left[b_{s}, u_{s}\right]\left(\phi_{s}(x)\right) d s\right) \rho(x) d x
$$

If we prove that, given $\rho \in C_{0}^{\infty}\left(\mathbb{R}^{d}\right)$ and $t \in[0, T]$, this $P$-a.s.-limit (which exists) is zero, then we have that $u$ is identically zero (because $\phi_{t}$ is a bijection).

Step 3. Let us check, by means of Corollary 19, that

$$
s \mapsto \int_{\mathbb{R}^{d}} \mathcal{R}_{\varepsilon}\left[b_{s}, u_{s}\right]\left(\phi_{s}(x)\right) \rho(x) d x
$$

satisfies the assumptions of Lebesgue dominated convergence theorem $(P$-a.s. $)$ on $[0, T]$. This will complete the proof.

Assume $\rho \in C_{r}^{\infty}\left(\mathbb{R}^{d}\right)$ for some $r>0$ and define the r.v. $R=\sup _{s \in[0, T], x \in B(r)}\left|\phi_{s}(x)\right|$ so that the maps $\phi_{s}$ send the support of $\rho$ strictly in the ball of radius $R$.

Let us give the details of the proof in the case $d>1$, the case $d=1$ being similar and easier. By Corollary 19 , for every $s \in[0, T]$ we have

$$
\begin{aligned}
& \left|\int \mathcal{R}_{\varepsilon}\left[b_{s}, u_{s}\right]\left(\phi_{s}(x)\right) \rho(x) d x\right| \\
& \quad \leq C_{\rho}\left\|u_{s}\right\|_{L_{R+1}^{\infty}}\left[\left\|\operatorname{div} b_{s}\right\|_{L_{R+1}^{1}}\left\|J \phi_{s}^{-1}\right\|_{L_{R}^{\infty}}+\left\|b_{s}\right\|_{L_{R+1}^{\infty}}\left(\left\|D \phi_{s}^{-1}\right\|_{L_{R}^{\infty}}+\left\|D J \phi_{s}^{-1}\right\|_{L_{R}^{1}}\right)\right] \\
& \quad \leq C_{\rho}\left[\left\|\operatorname{div} b_{s}\right\|_{L_{R+1}^{1}}\left\|J \phi_{s}^{-1}\right\|_{L_{R}^{\infty}}+\left\|D \phi_{s}^{-1}\right\|_{L_{R}^{\infty}}+\left\|D J \phi_{s}^{-1}\right\|_{L_{R}^{1}}\right]
\end{aligned}
$$

by the global boundedness of $b$ and $u$. From the properties of the stochastic flow $\phi$ we know that $P(R<\infty)=1$ and that $(s, x) \mapsto D \phi_{s}^{-1}(x)$ is $P$-a.s. continuous. Hence the term $\sup _{s \in[0, T]}\left\|J \phi_{s}^{-1}\right\|_{L_{R}^{\infty}}$ and $\sup _{s \in[0, T]}\left\|D \phi_{s}^{-1}\right\|_{L_{R}^{\infty}}$ are $P$-a.s. finite. Moreover, $\int_{0}^{T}\left\|\operatorname{div} b_{s}\right\|_{L_{R+1}^{1}} d s<$ $\infty$. So it remains to show that

$$
\int_{0}^{T}\left\|D J \phi_{s}^{-1}\right\|_{L_{R}^{1}} d s<\infty, \quad P \text {-a.s. }
$$

where $R$ is a positive r.v. which is $P$-a.s. finite. This bound will follow from a similar bound where $R$ is replaced by an arbitrary positive number. Moreover, since by Lemma 7 the equation 
for $\phi_{s}^{-1}$ is equal to the equation for $\phi_{s}$ (up to a sign and inversion of time) we can use Theorem 11 to conclude. The proof is complete.

Let us formulate our second main result which basically only requires Hypothesis 1 but with $\alpha>1 / 2$ (for any $d \geq 1$ ). Here we do not need the regularity results on the derivatives of $J \phi$ in Sobolev spaces.

Theorem 21 Assume that Hypothesis 1 holds true with $\alpha>1 / 2$. Moreover assume that $\operatorname{div} b \in$ $L_{\mathrm{loc}}^{1}\left([0, T] \times \mathbb{R}^{d}\right)$. Then, for every $u_{0} \in L^{\infty}$, there exists a unique weak $L^{\infty}$-solution of the Cauchy problem (11) which has the form $u(t, x, \omega)=u_{0}\left(\phi_{t}^{-1}(\omega) x\right)$.

The proof requires the following lemma, in which we provide a special bound for the commutator.

Lemma 22 Given $v \in L_{l o c}^{\infty}\left(\mathbb{R}^{d}, \mathbb{R}^{d}\right)$, $\operatorname{div} v \in L_{l o c}^{1}\left(\mathbb{R}^{d}\right), g \in L_{l o c}^{\infty}\left(\mathbb{R}^{d}\right)$.

(i) For any $\rho \in C_{r}^{\infty}\left(\mathbb{R}^{d}\right)$ and for sufficiently small $\varepsilon$ we have, for some positive constant $C_{r}$,

$$
\begin{array}{r}
\left|\int \mathcal{R}_{\varepsilon}[g, v](x) \rho(x) d x\right| \leq C_{r}\|g\|_{L_{r+1}^{\infty}}\|\rho\|_{L_{r}^{\infty}}\|\operatorname{div} v\|_{L_{r+1}^{1}} \\
+\left|\iint g\left(x^{\prime}\right) D_{x} \vartheta_{\varepsilon}\left(x-x^{\prime}\right)\left(\rho(x)-\rho\left(x^{\prime}\right)\right)\left[v(x)-v\left(x^{\prime}\right)\right] d x d x^{\prime}\right| .
\end{array}
$$

(ii) If in addition there exists $\theta \in(0,1)$ such that $v \in C_{\mathrm{loc}}^{\theta}\left(\mathbb{R}^{d}, \mathbb{R}^{d}\right)$, then we have the uniform bound

$$
\left|\int \mathcal{R}_{\varepsilon}[g, v](x) \rho(x) d x\right| \leq C_{r}\|g\|_{L_{r+1}^{\infty}}\left(\|\rho\|_{L_{r}^{\infty}}\|\operatorname{div} v\|_{L_{r+1}^{1}}+[v]_{C_{r+1}^{\theta}}[\rho]_{C_{r+1}^{1-\theta}}\right) .
$$

Proof. (i) We start as in the proof of Lemma 18, We write

$$
\int \mathcal{R}_{\varepsilon}[g, v](x) \rho(x) d x=J_{1}(\rho)+J_{2}(\rho)
$$

where

$$
\begin{gathered}
J_{1}(\rho)=\iint g\left(x^{\prime}\right) \vartheta_{\varepsilon}\left(x-x^{\prime}\right) D_{x} \rho(x)\left[v(x)-v\left(x^{\prime}\right)\right] d x d x^{\prime} \\
J_{2}(\rho)=\iint g\left(x^{\prime}\right) \rho(x) \vartheta_{\varepsilon}\left(x-x^{\prime}\right)\left[\operatorname{div} v(x)-\operatorname{div} v\left(x^{\prime}\right)\right] d x d x^{\prime} .
\end{gathered}
$$

Let us estimate $J_{2}$. By changing variables, $x=\varepsilon y+y^{\prime}, x^{\prime}=y^{\prime}$,

$$
\begin{gathered}
J_{2}(\rho)=\frac{1}{\varepsilon^{d}} \iint g\left(x^{\prime}\right) \rho(x) \vartheta\left(\frac{x-x^{\prime}}{\varepsilon}\right)\left[\operatorname{div} v(x)-\operatorname{div} v\left(x^{\prime}\right)\right] d x d x^{\prime} \\
=\iint g\left(y^{\prime}\right) \rho\left(\varepsilon y+y^{\prime}\right) \vartheta(y)\left[\operatorname{div} v\left(\varepsilon y+y^{\prime}\right)-\operatorname{div} v\left(y^{\prime}\right)\right] d y d y^{\prime}
\end{gathered}
$$

Hence

$$
\begin{gathered}
\left|J_{2}(\rho)\right| \leq\|g\|_{L_{r+1}^{\infty}}\|\rho\|_{L_{r}^{\infty}} \iint\left(\left|\operatorname{div} v\left(\varepsilon y+y^{\prime}\right)\right|+\left|\operatorname{div} v\left(y^{\prime}\right)\right|\right) d y d y^{\prime} \\
\leq C_{r}\|g\|_{L_{r+1}^{\infty}}\|\rho\|_{L_{r}^{\infty}}\|\operatorname{div} v\|_{L_{r+1}^{1}}
\end{gathered}
$$


In order to estimate $J_{1}$, we note that

$$
\begin{gathered}
J_{1}(\rho)=\iint g\left(x^{\prime}\right) \vartheta_{\varepsilon}\left(x-x^{\prime}\right) D_{x}\left(\rho(x)-\rho\left(x^{\prime}\right)\right)\left[v(x)-v\left(x^{\prime}\right)\right] d x d x^{\prime} \\
=-\iint g\left(x^{\prime}\right) D_{x} \vartheta_{\varepsilon}\left(x-x^{\prime}\right)\left(\rho(x)-\rho\left(x^{\prime}\right)\right)\left[v(x)-v\left(x^{\prime}\right)\right] d x d x^{\prime} \\
-\iint g\left(x^{\prime}\right) \vartheta_{\varepsilon}\left(x-x^{\prime}\right)\left(\rho(x)-\rho\left(x^{\prime}\right)\right) \operatorname{div} v(x) d x d x^{\prime} \\
=J_{11}(\rho)+J_{12}(\rho) .
\end{gathered}
$$

Let us treat $J_{12}(\rho)$. We find

$$
\left|J_{12}(\rho)\right| \leq 2\|g\|_{L_{r+1}^{\infty}}\|\rho\|_{L_{r+1}^{\infty}} \int|\operatorname{div} v(x)| d x \int \vartheta_{\varepsilon}\left(x-x^{\prime}\right) d x^{\prime} \leq 2\|g\|_{L_{r+1}^{\infty}}\|\rho\|_{L_{r+1}^{\infty}}\|\operatorname{div} v\|_{L_{r+1}^{1}} .
$$

(ii) We only have to estimate $J_{11}(\rho)$. We get

$$
\begin{aligned}
\left|J_{11}(\rho)\right| & \leq\left|\frac{1}{\varepsilon^{d+1}} \iint g\left(x^{\prime}\right) D_{x} \vartheta\left(\frac{x-x^{\prime}}{\varepsilon}\right)\left(\rho(x)-\rho\left(x^{\prime}\right)\right)\left[v(x)-v\left(x^{\prime}\right)\right] d x d x^{\prime}\right| \\
& \leq \frac{1}{\varepsilon}[v]_{C_{r+1}^{\theta}}[\rho]_{C_{r+1}^{1-\theta}}\|g\|_{L_{r+1}^{\infty}} \frac{1}{\varepsilon^{d}} \iint\left|D_{x} \vartheta\left(\frac{x-x^{\prime}}{\varepsilon}\right)\right|\left|x-x^{\prime}\right| d x d x^{\prime} \\
& \leq \frac{1}{\varepsilon^{d}} \iint\left|D_{x} \vartheta\left(\frac{x-x^{\prime}}{\varepsilon}\right)\right| d x d x^{\prime} \leq C[v]_{C_{r+1}^{\theta}}[\rho]_{C_{r+1}^{1-\theta}}\|g\|_{L_{r+1}^{\infty}},
\end{aligned}
$$

where $C$ is independent on $\varepsilon$. The proof is complete.

The previous result is now extended to the case in which commutators are composed with a flow.

Corollary 23 Let $\phi$ be a $C^{1}$-diffeomorphism of $\mathbb{R}^{d}$ (J申 denotes its Jacobian). Assume that there exists $\theta \in(0,1)$ such that $v \in C_{\mathrm{loc}}^{\theta}\left(\mathbb{R}^{d}, \mathbb{R}^{d}\right)$, div $v \in L_{l o c}^{1}\left(\mathbb{R}^{d}\right), g \in L_{l o c}^{\infty}\left(\mathbb{R}^{d}\right)$. Moreover assume that $J \phi \in C_{\mathrm{loc}}^{1-\theta}\left(\mathbb{R}^{d}\right)$. Then, for any $\rho \in C_{r}^{\infty}\left(\mathbb{R}^{d}\right)$ and any $R>0$ such that $\operatorname{supp}\left(\rho \circ \phi^{-1}\right) \subseteq B(R)$, we have the uniform bound

$$
\begin{aligned}
& \left|\int \mathcal{R}_{\varepsilon}[g, v](\phi(x)) \rho(x) d x\right| \leq C_{r}\|g\|_{L_{r+1}^{\infty}}\|\rho\|_{L_{r}^{\infty}}\left\|J \phi^{-1}\right\|_{L_{R}^{\infty}}\|\operatorname{div} v\|_{L_{r+1}^{1}} \\
& +C_{r}\|g\|_{L_{r+1}^{\infty}}[v]_{C_{r+1}^{\theta}}\left(\left\|D \phi^{-1}\right\|_{C_{R}^{1-\theta}}\|D \rho\|_{L_{r}^{\infty}}+\|\rho\|_{L_{r}^{\infty}}\left[D \phi^{-1}\right]_{C_{R}^{1-\theta}}\right) .
\end{aligned}
$$

In addition,

$$
\lim _{\varepsilon \rightarrow 0} \int \mathcal{R}_{\varepsilon}[g, v](\phi(x)) \rho(x) d x=0
$$

Proof. By changing variable, we have $\int \mathcal{R}_{\varepsilon}[g, v](\phi(x)) \rho(x) d x=\int \mathcal{R}_{\varepsilon}[g, v](y) \rho_{\phi}(y) d x$ where the function

$$
\rho_{\phi}(y)=\rho\left(\phi^{-1}(y)\right) J \phi^{-1}(y)
$$

has the support strictly contained in the ball of radius $R$. Clearly, $\left\|\rho_{\phi}\right\|_{L_{R}^{\infty}} \leq\|\rho\|_{L_{r}^{\infty}}\left\|J \phi^{-1}\right\|_{L_{R}^{\infty}}$. 
To prove the result, we have to check that Lemma 22 can be applied with $\rho_{\phi}$ instead of $\rho$. This follows since

$$
\begin{gathered}
{\left[\rho_{\phi}\right]_{C_{R}^{1-\theta}} \leq\left\|J \phi^{-1}\right\|_{L_{R}^{\infty}}\left[\rho\left(\phi^{-1}(\cdot)\right)\right]_{C_{R}^{1-\theta}}+\|\rho\|_{L_{r}^{\infty}}\left[J \phi^{-1}\right]_{C_{R}^{1-\theta}}} \\
\leq\left\|D \phi^{-1}\right\|_{L_{R}^{\infty}}\|D \rho\|_{L_{r}^{\infty}}\left[D \phi^{-1}\right]_{C_{R}^{1-\theta}}+\|\rho\|_{L_{r}^{\infty}}\left[D \phi^{-1}\right]_{C_{R}^{1-\theta}} .
\end{gathered}
$$

Proof of Theorem 21. We follow the proof of Theorem 20, The first two steps are just the same. The only change is in Step 3.

Step 3. We have to check that

$$
s \mapsto \int_{\mathbb{R}^{d}} \mathcal{R}_{\varepsilon}\left[u_{s}, b_{s}\right]\left(\phi_{s}(x)\right) \rho(x) d x
$$

satisfies the assumptions of Lebesgue dominated convergence theorem (P-a.s. $)$ on $[0, T]$. Assume $\rho \in C_{r}^{\infty}\left(\mathbb{R}^{d}\right)$ for some $r>0$ and define the random variable $R=\sup _{s \in[0, T], x \in B(r)}\left|\phi_{s}(x)\right|$ so that the maps $\phi_{s}$ send the support of $\rho$ strictly in the ball of radius $R$. Note that

$$
\sup _{s \in[0, T], x \in B(R)}\left|J \phi_{s}^{-1}(x)\right|<\infty .
$$

Recall that $D \phi_{s}^{-1}$ is $P$-a.s. locally $\alpha^{\prime}$-Holder continuous, uniformly in $s \in[0, T]$, for any $\alpha^{\prime} \in$ $(0, \alpha)$. Since $\alpha>1 / 2$, we infer by Corollary 23 with $\theta=1 / 2$

$$
\left|\int \mathcal{R}_{\varepsilon}\left[u_{s}, b_{s}\right]\left(\phi_{s}(x)\right) \rho(x) d x\right| \leq C_{\rho}\left\|u_{s}\right\|_{L_{R}^{\infty}}\left(\left\|\operatorname{div} b_{s}\right\|_{L_{R+1}^{1}}+\left[b_{s}\right]_{C_{R+1}^{1 / 2}}\left\|D \phi_{s}^{-1}\right\|_{C_{R}^{1 / 2}}\right) .
$$

From the properties of the stochastic flow $\phi$ we know that $P(R<\infty)=1$ and that $(s, x) \mapsto$ $D \phi_{s}^{-1}(x)$ is $P$-a.s. continuous. Hence the terms $\int_{0}^{T}\left\|\operatorname{div} b_{s}\right\|_{L_{R}^{1}} d s$ and $\sup _{s \in[0, T]}\left(\left[b_{s}\right]_{C_{R+1}^{1 / 2}}\left\|D \phi_{s}^{-1}\right\|_{C_{R}^{1 / 2}}\right)$ are $P$-a.s. finite and so we can apply the dominated convergence theorem. The proof is complete.

\section{$6 \quad$ Examples}

In this section we give two classes of examples. First, we recall a classical example of nonuniqueness for the deterministic transport equation and we observe the improvements obtained by random perturbation (we call it a 'positive example'). Notice however that other relevant examples of deterministic non-uniqueness, like the one of N. Depauw [9], are not covered by the results of our work since there $b$ is not Hölder continuous in the space variable.

Second, we show by means of two 'negative examples' that it is not clear how to extend the approach of this paper to random fields $b$ and nonlinear SPDEs.

\subsection{A positive example}

Without noise, the transport equation with an Hölder vector field is not necessarily well-posed. A counter-example can be easily constructed in 1d. Consider the function

$$
b(x)=\frac{1}{1-\gamma} \operatorname{sign}(x)(|x| \wedge R)^{\gamma}, \quad \gamma \in(0,1)
$$


(the simplest but less symmetric case $b(x)=\frac{1}{1-\gamma}(|x| \wedge R)^{\gamma}$ is similar, and many other variants can be treated in the same way), where $R>0$ is introduced only to have boundedness. This function is $C_{b}^{\gamma}(\mathbb{R})$ and $\operatorname{div} b=b^{\prime} \in L^{p}(\mathbb{R})$ for all $p \in(1,1 /(1-\gamma))$. Hence it satisfies hypothesis 1 for every $\gamma \in(0,1)$, hypothesis 2 for $\gamma \in\left(\frac{1}{2}, 1\right)$ (because of the restriction $p>2$ ) and the condition $D b \in L_{l o c}^{1}([0, T] \times \mathbb{R})$ for every $\gamma \in(0,1)$. Hence the stochastic flow of diffeomorphisms exists and the stochastic transport equation is well posed in $L^{\infty}$ and in $B V_{\text {loc }}$ (see Appendix A below) for all $\gamma \in(0,1)$.

On the contrary, the deterministic transport equation is not well posed; let us recall why. The Cauchy problem

$$
x^{\prime}(t)=b(x(t)), \quad t \geq 0, \quad x(0)=x_{0}
$$

has a unique solution for all $x_{0} \neq 0$, denoted by $\phi_{t}\left(x_{0}\right)$. For $x_{0}=0$ we have two extremal solutions $x_{+}(t)$ e $x_{-}(t), x_{+}(t)=t^{\frac{1}{1-\gamma}}$ and $x_{-}(t)=-t^{\frac{1}{1-\gamma}}$ for small $t$. In addition, for $x_{0}=0$, we have the solution $x(t) \equiv 0$, and the solutions $x(t)=x_{ \pm}\left(t-t_{0}\right) 1_{t \geq t_{0}}$ for every $t_{0} \geq 0$. Given $t>$ 0 and $x \in\left[x_{-}(t), x_{+}(t)\right]$, there is a unique number $t_{0}(t, x) \geq 0$ such that $x_{\operatorname{sign}(x)}\left(t-t_{0}(t, x)\right)=$ $x$. The function $\phi_{t}$ maps $\mathbb{R} \backslash\{0\}$ one to one on $\left(-\infty, x_{-}(t)\right) \cup\left(x_{+}(t), \infty\right) ; \phi_{t}^{-1}$ will be its inverse, between these sets. With these notations, given $u_{0} \in L^{\infty}$ and two bounded measurable functions $\gamma_{+}, \gamma_{-}:[0, \infty) \rightarrow \mathbb{R}$, define the function

$$
u_{\gamma_{ \pm}}(t, x)=\left\{\begin{array}{ccc}
u_{0}\left(\phi_{t}^{-1}(x)\right) & \text { for } & x>x_{+}(t) \\
\gamma_{+}\left(t_{0}(t, x)\right) & \text { for } & 0 \leq x \leq x_{+}(t) \\
\gamma_{-}\left(t_{0}(t, x)\right) & \text { for } & x_{-}(t) \leq x<0 \\
u_{0}\left(\phi_{t}^{-1}(x)\right) & \text { for } & x<x_{-}(t)
\end{array} .\right.
$$

These are weak $L^{\infty}$ solutions, for every $\gamma_{+}, \gamma_{-}$, of the deterministic transport equation with the same initial condition $u_{0}$. For instance, if $u_{0}=1_{x>0}$ and $\gamma_{+}=\gamma_{-} \equiv a$ for a constant value $a$, the shape of $u_{\gamma_{ \pm}}$can be easily worked out. All these functions are solutions both in $L^{\infty}$ and in $B V_{\text {loc }}$, corresponding to the same $B V_{\text {loc }}$ initial condition $u_{0}$.

\subsection{Negative examples}

It would be interesting to generalize the results of this paper to random vectorfields $b(t, x, \omega)$, possibly adapted. However our approach faces a fundamental difficulty: it is very easy to exhibit a counterexample which shows that in some cases the regularizing effect disappears. Consider in one dimension the case

$$
b(t, x, \omega)=\sqrt{\left|x-W_{t}(\omega)\right|},
$$

namely the stochastic differential equation

$$
d X_{t}^{x}=b\left(t, X_{t}^{x}, \cdot\right) d t+d W_{t}, \quad t \geq 0, \quad X_{0}^{x}=x .
$$

If $\left(X_{t}^{x}\right)$ is a solution, then $Y_{t}^{x}=X_{t}^{x}-W_{t}$ solves

$$
d Y_{t}^{x}=\sqrt{\left|Y_{t}^{x}\right|} d t \quad t \geq 0, \quad Y_{0}^{x}=x
$$

and viceversa. Hence the non-uniqueness for the latter equation transfer to the former. In terms of stochastic transport equation, an equation of the form

$$
\partial_{t} u(t, x)+\left(b_{0}(x-W(t)) \cdot D u(t, x)\right) d t+D u(t, x) \circ d W(t)=0
$$


may have several pathologies if $b_{0}$ is only Hölder continuous.

Unfortunately the previous example is not so artificial: something similar happens in the nonlinear case. Let us argue only formally. Consider the example in $\mathbb{R}^{2}$

$$
\partial_{t} \xi(t, x)+(u(t, x) \cdot D \xi(t, x)) d t+\sum_{i=1}^{d} D_{i} \xi(t, x) \circ d W^{i}(t)=0
$$

where $\xi=\partial_{2} u_{1}-\partial_{1} u_{2}$. This is the vorticity equation of a $2 \mathrm{D}$ ideal fluid described by a stochastic version of Euler equation. Following [30, this equation is (formally) equivalent to the family of stochastic ordinary equations depending on a parameter $a \in \mathbb{R}^{2}$

$$
d X_{t}^{a}=\left[\int_{\mathbb{R}^{2}} K\left(X_{t}^{a}-X_{t}^{a^{\prime}}\right) \xi_{0}\left(X_{t}^{a^{\prime}}\right) d a^{\prime}\right] d t+d W_{t}
$$

for a suitable kernel $K, \xi_{0}$ being the initial condition of the vorticity equation. This problem is equivalent to

$$
d Y_{t}^{a}=\left[\int_{\mathbb{R}^{2}} K\left(Y_{t}^{a}-Y_{t}^{a^{\prime}}\right) \xi_{0}\left(X_{t}^{a^{\prime}}\right) d a^{\prime}\right] d t
$$

by the change of variable $Y_{t}^{a}=X_{t}^{a}-W_{t}$, and the equation for $\left(Y_{t}^{a}\right)$ corresponds to the classical vorticity equation

$$
\frac{\partial_{t} \xi^{\prime}(t, x)}{\partial t}+\left(u^{\prime}(t, x) \cdot D \xi^{\prime}(t, x)\right) d t=0 \quad \xi^{\prime}=\partial_{2} u_{1}^{\prime}-\partial_{1} u_{2}^{\prime}
$$

with initial condition $\xi_{0}$. This means that the stochastic vorticity equation is (at least formally) equivalent to the deterministic one. There is no advantage to introduce that kind of stochastic perturbation.

\section{A Existence and uniqueness in $B V_{l o c}$}

The results proved in Section 2 on the stochastic flow allow one to prove several existence and uniqueness results in spaces more regular than $L^{\infty}$. We describe here the case $B V_{l o c}$, as a less trivial example. Let us emphasize that only the assumption $b \in L^{\infty}\left(0, T ; C_{b}^{\alpha}\left(\mathbb{R}^{d} ; \mathbb{R}^{d}\right)\right)$ is needed; no condition on $\operatorname{div} b$ is imposed. This is again at variance with the deterministic case.

For more details on the functions of locally bounded variation see 14. Let us recall that a function $v \in L_{l o c}^{1}\left(\mathbb{R}^{d}\right)$ is said to be of locally bounded variation, $v \in B V_{l o c}\left(\mathbb{R}^{d}\right)$ if its distributional derivatives $D_{i} v, i=1, \ldots, d$, are signed Radon measures. We denote by $D v$ the vector valued measure with components $D_{i} v$. We have

$$
\int_{\mathbb{R}^{d}} \vartheta(x) \cdot D v(d x)=-\int_{\mathbb{R}^{d}} v(x) \operatorname{div} \vartheta(x) d x
$$

for all vector fields $\vartheta \in C_{0}^{1}\left(\mathbb{R}^{d}, \mathbb{R}^{d}\right)$, and also for all $\vartheta \in C_{0}\left(\mathbb{R}^{d}, \mathbb{R}^{d}\right)$ such that the distributional divergence (which has compact support) div $\vartheta$ is of class $L^{1}\left(\mathbb{R}^{d}\right)$.

When $a \in C\left(\mathbb{R}^{d}, \mathbb{R}^{d}\right)$ is a given vector field and $v \in B V_{l o c}\left(\mathbb{R}^{d}\right)$, the notation $a \cdot D v$ stands for the (scalar) distribution $\theta \mapsto \int_{\mathbb{R}^{d}} \theta(x) a(x) \cdot D v(d x), \theta \in C_{0}\left(\mathbb{R}^{d}\right)$. This is the meaning of the notation $\int_{\mathbb{R}^{d}} \theta(x) b_{s}(x) \cdot D u_{s}(d x)$ used in the next definition. 
We denote by $L_{\mathcal{F}}^{\infty}\left(B V_{l o c}\left(\mathbb{R}^{d}\right)\right)$ the space of all stochastic processes $u \in L^{\infty}\left(\Omega \times[0, T] \times \mathbb{R}^{d}\right)$ such that $u(\omega, t, \cdot) \in B V_{l o c}\left(\mathbb{R}^{d}\right)$ for a.e. $(\omega, t) \in \Omega \times[0, T]$, for every $\theta \in C_{0}^{\infty}\left(\mathbb{R}^{d}\right)$ and $\vartheta \in$ $L^{\infty}\left(0, T ; C_{0}\left(\mathbb{R}^{d}, \mathbb{R}^{d}\right)\right)$ the processes

$$
\int_{\mathbb{R}^{d}} \theta(x) u(t, x) d x, \quad \int_{\mathbb{R}^{d}} \vartheta(t, x) \cdot D u(t, d x)
$$

are progressively measurable with respect to $\left(\mathcal{F}_{t}\right)_{t \in[0, T]}$, and $|D u(\omega, t)|$ (the total variation of the measure $D u(\omega, t, \cdot))$ has the following property:

$$
\int_{0}^{T}|D u(\omega, t)|(B(r)) d t<\infty
$$

for all $r>0$, for $P$-a.e. $\omega \in \Omega$. We use again the notation $u_{t}$ for $u(t, \cdot)$ below.

Definition 24 Let $b \in L^{\infty}\left(0, T ; C_{b}^{\alpha}\left(\mathbb{R}^{d} ; \mathbb{R}^{d}\right)\right)$ and $u_{0} \in B V_{\text {loc }}\left(\mathbb{R}^{d}\right)$. A stochastic process $u \in$

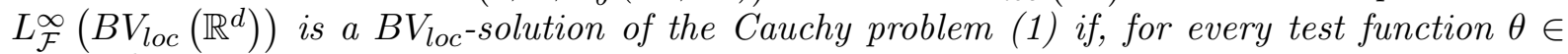
$C_{0}^{\infty}\left(\mathbb{R}^{d}\right)$, the process $\int_{\mathbb{R}^{d}} \theta(x) u_{t}(x) d x$ has a continuous modification which is an $\mathcal{F}$-semimartingale and

$$
\begin{aligned}
\int_{\mathbb{R}^{d}} u_{t}(x) \theta(x) d x & =\int_{\mathbb{R}^{d}} u_{0}(x) \theta(x) d x \\
& -\int_{0}^{t}\left(\int_{\mathbb{R}^{d}} \theta(x) b_{s}(x) \cdot D u_{s}(d x)\right) d s \\
& +\sum_{i=1}^{d} \int_{0}^{t}\left(\int_{\mathbb{R}^{d}} u_{s}(x) D_{i} \theta(x) d x\right) \circ d W_{s}^{i} .
\end{aligned}
$$

If $u$ is a $B V_{l o c}$-solution and $\operatorname{div} b \in L_{l o c}^{1}\left([0, T] \times \mathbb{R}^{d}\right)$, then $u$ is also a weak $L^{\infty}$-solution. Conversely, if $u$ is a weak $L^{\infty}$-solution, $u_{0} \in B V_{l o c}\left(\mathbb{R}^{d}\right)$ and $u \in L_{\mathcal{F}}^{\infty}\left(B V_{l o c}\left(\mathbb{R}^{d}\right)\right)$, then $u$ is a $B V_{l o c}$-solution.

Theorem 25 If $b \in L^{\infty}\left(0, T ; C_{b}^{\alpha}\left(\mathbb{R}^{d} ; \mathbb{R}^{d}\right)\right)$ and $u_{0} \in B V_{\text {loc }}\left(\mathbb{R}^{d}\right)$, there exists a unique $B V_{\text {loc }}{ }^{-}$ solution $u$, given by $u(t, x)=u_{0}\left(\phi_{t}^{-1}(x)\right)$.

Proof. Step 1 (Existence). Let us mention a preliminary known fact. If $u_{0} \in B V_{l o c}\left(\mathbb{R}^{d}\right)$ and $\varphi$ is a diffeomorphism of $\mathbb{R}^{d}$ (differentiable in both directions with continuous derivatives), then $u_{0} \circ \varphi \in B V_{l o c}\left(\mathbb{R}^{d}\right)$ and the signed Radon measures $D_{i}\left(u_{0} \circ \varphi\right)(d x), i=1, \ldots, d$, are defined by

$$
\int_{\mathbb{R}^{d}} \theta(x) D_{i}\left(u_{0} \circ \varphi\right)(d x)=\int_{\mathbb{R}^{d}} \theta\left(\varphi^{-1}(y)\right) J \varphi^{-1}(y)\left(D_{i} \varphi\right)\left(\varphi^{-1}(y)\right) \cdot D u_{0}(d y)
$$

for every $\theta \in C_{0}^{\infty}\left(\mathbb{R}^{d}\right)$. To prove this claim we use the characterization of $B V_{l o c}$ functions $v$ as the weak $L_{l o c}^{1}\left(\mathbb{R}^{d}\right)$ limits of $W_{l o c}^{1,1}\left(\mathbb{R}^{d}\right)$ (or $\left.C_{0}^{\infty}\left(\mathbb{R}^{d}\right)\right)$ functions $v_{n}$ such that $\sup _{n} \int_{B(r)}\left|D v_{n}(x)\right| d x<$ $\infty$ for every $r>0$ (see [14], Chapter 4). Take a sequence of functions $u_{0}^{n} \in C_{0}^{\infty}\left(\mathbb{R}^{d}\right)$ such that $u_{0}^{n} \rightarrow u_{0}$ in $L_{l o c}^{1}\left(\mathbb{R}^{d}\right), \sup _{n} \int_{B(r)}\left|D u_{0}^{n}(x)\right| d x<\infty$ for every $r>0$. The function $u_{0}^{n} \circ \varphi$ is in $C_{0}^{1}\left(\mathbb{R}^{d}\right), u_{0}^{n} \circ \varphi \rightarrow u_{0} \circ \varphi$ in $L_{l o c}^{1}\left(\mathbb{R}^{d}\right)$ and

$$
\begin{aligned}
\int_{B(r)}\left|D_{i}\left(u_{0}^{n} \circ \varphi\right)(x)\right| d x & \leq \int_{B(r)}\left|D u_{0}^{n}(\varphi(x)) \cdot D_{i} \varphi(x)\right| d x \\
& =\int_{\varphi(B(r))}\left|D u_{0}^{n}(y) \cdot D_{i} \varphi\left(\varphi^{-1}(y)\right)\right| J \varphi^{-1}(y) d y
\end{aligned}
$$


which implies that $\sup _{n} \int_{B(r)}\left|D\left(u_{0}^{n} \circ \varphi\right)(x)\right| d x<\infty$ for every $r>0$ and thus $u_{0} \circ \varphi \in$ $B V_{l o c}\left(\mathbb{R}^{d}\right)$. Similarly

$$
\int_{\mathbb{R}^{d}} \theta(x) D_{i}\left(u_{0}^{n} \circ \varphi\right)(d x)=\int_{\mathbb{R}^{d}} \theta\left(\varphi^{-1}(y)\right) J \varphi^{-1}(y) D_{i} \varphi\left(\varphi^{-1}(y)\right) \cdot D u_{0}^{n}(y) d y .
$$

Up to a common subsequence, the measures $D_{i}\left(u_{0}^{n} \circ \varphi\right)$ and $D u_{0}^{n}$ weakly converge to $D_{i}\left(u_{0} \circ \varphi\right)$ and $D u_{0}$ respectively, on $B(r)$ for every $r>0$ (Proposition 5 of [14], Chapter 4.1.1). We can take the limit in the previous identity and get (42).

Let us prove the existence claim. Let $\phi_{t}$ be the stochastic flow of diffeomorphisms given under the assumption $b \in L^{\infty}\left(0, T ; C_{b}^{\alpha}\left(\mathbb{R}^{d} ; \mathbb{R}^{d}\right)\right)$ and let us set $u(t, x)=u_{0}\left(\phi_{t}^{-1}(x)\right)$. For every set $A \subset \mathbb{R}^{d}$, denote by $\phi(\omega, A)$ the image of $[0, T] \times A$ under the mapping $(t, x) \mapsto \phi_{t}(\omega, x)$. This mapping is $P$-a.s. continuous, hence $\phi(\omega, B)$ is a bounded set $P$-a.s., for every bounded set $B$.

We have $u \in L^{\infty}\left(\Omega \times[0, T] \times \mathbb{R}^{d}\right)$ and $\int_{\mathbb{R}^{d}} \theta(x) u(t, x) d x$ is progressively measurable for every $\theta \in C_{0}^{\infty}\left(\mathbb{R}^{d}\right)$. Since $u_{0} \in B V_{\text {loc }}\left(\mathbb{R}^{d}\right)$, for a.e. $\omega \in \Omega$ we have that for all $t \in[0, T]$ the function $x \mapsto u_{0}\left(\phi_{t}^{-1}(x)\right)$ belongs to $B V_{l o c}\left(\mathbb{R}^{d}\right)$ and, for $\theta \in L^{\infty}\left(0, T ; C_{0}\left(\mathbb{R}^{d}\right)\right)$ and $i=1, \ldots, d$,

$$
\int_{\mathbb{R}^{d}} \theta_{t}(x) D_{i} u_{t}(d x)=\int_{\mathbb{R}^{d}} \theta_{t}\left(\phi_{t}^{-1}(y)\right) J \phi_{t}^{-1}(y)\left(D_{i} \phi_{t}\right)\left(\phi_{t}^{-1}(y)\right) \cdot D u_{0}(d y)
$$

which is progressively measurable. Moreover, since $\left|D_{i} u_{t}\right|(B(r))$ is the supremum of the quantity $\left|\int_{B(r)} \theta(x) D_{i} u_{t}(d x)\right|$ over all $\theta \in C_{0}^{1}(B(r))$ such that $|\theta(x)| \leq 1$ for all $x \in B(r)$, and from the previous identity we get

$$
\begin{aligned}
\left|\int_{B(r)} \theta(x) D_{i} u_{t}(d x)\right| & \leq \int_{\phi_{t}(B(r))} J \phi_{t}^{-1}(y)\left|\left(D_{i} \phi_{t}\right)\left(\phi_{t}^{-1}(y)\right)\right|\left|D u_{0}\right|(d y) \\
& \leq\left|D u_{0}\right|(\phi(\omega, B(r)))\left\|J \phi_{t}^{-1}(\cdot)\left|\left(D_{i} \phi_{t}\right)\left(\phi_{t}^{-1}(\cdot)\right)\right|\right\|_{L^{\infty}(\phi(\omega, B(r)))}
\end{aligned}
$$

we deduce (41) and even $P\left(\sup _{t \in[0, T]}\left|D u_{t}\right|(B(r))<\infty\right)=1$. Hence $u \in L_{\mathcal{F}}^{\infty}\left(B V_{\text {loc }}\left(\mathbb{R}^{d}\right)\right)$.

We may now repeat the proof of Theorem 16 and prove that $u(t, x)=u_{0}\left(\phi_{t}^{-1}(x)\right)$ is a solution; the difference is that in Section 4 we assumed $\operatorname{div} b \in L_{l o c}^{1}$ and $u$ was only $L^{\infty}$, while here $\operatorname{div} b$ is only a distribution but $u \in B V_{l o c}\left(\mathbb{R}^{d}\right)$, so we have to write differently the integrals involving $b \cdot D u$.

Step 2 (Uniqueness). We repeat the first part of the proof of Theorem 23: by linearity, we treat the case $u_{0}=0$; we mollify a $B V_{l o c}$-solution $u \in L_{\mathcal{F}}^{\infty}\left(B V_{l o c}\left(\mathbb{R}^{d}\right)\right)$, apply stochastic calculus and prove the same results as in steps 1 and 2 of the proof of Theorem 23. The commutator $\mathcal{R}_{\varepsilon}\left[u_{s}, b_{s}\right]$ is always given by $\mathcal{R}_{\varepsilon}\left[u_{s}, b_{s}\right]=\vartheta_{\varepsilon} *\left(b_{s} \cdot D u_{s}\right)-b_{s} \cdot D\left(\vartheta_{\varepsilon} * u_{s}\right)$, but here we stress that $b_{s} \cdot D u_{s}$ is the distribution having the meaning recalled at the beginning of the section (we have to use compact support mollifiers). We have to prove that, $P$-a.s.,

$$
\lim _{\varepsilon \rightarrow 0} \int_{0}^{t}\left(\int \mathcal{R}_{\varepsilon}\left[u_{s}, b_{s}\right]\left(\phi_{s}(x)\right) \rho(x) d x\right) d s=0
$$

for every $\rho \in C_{0}^{\infty}\left(\mathbb{R}^{d}\right)$, namely step 3 of the proof of Theorem 23 . Equivalently, we have to prove that $P$-a.s.

$$
\lim _{\varepsilon \rightarrow 0} \int_{0}^{t}\left(\int \mathcal{R}_{\varepsilon}\left[u_{s}, b_{s}\right](y) \theta_{s}(y) d y\right) d s=0
$$


where $\theta_{t}(x)=\rho\left(\phi_{t}^{-1}(x)\right) J \phi_{t}^{-1}(x)$. This is, $P$-a.s., a bounded continuous function of $(t, x)$, and $\theta_{t}(\omega, x)=0$ for all $x \notin \phi(\omega, K)$ and all $t \in[0, T]$, where $K$ is the support of $\rho$. One has

$$
\mathcal{R}_{\varepsilon}\left[u_{s}, b_{s}\right](y)=\int \vartheta_{\varepsilon}(y-z)\left[b_{s}(z)-b_{s}(y)\right] \cdot D u_{s}(d z)
$$

hence (all functions are bounded so we may apply Fubini theorem)

$$
\int_{0}^{t}\left(\int \mathcal{R}_{\varepsilon}\left[u_{s}, b_{s}\right](y) \theta_{s}(y) d y\right) d s=\int_{0}^{t}\left(\int f_{\varepsilon}(s, z) \cdot D u_{s}(d z)\right) d s
$$

where

$$
f_{\varepsilon}(\omega, s, z)=\int \vartheta_{\varepsilon}(y-z)\left[b_{s}(z)-b_{s}(y)\right] \theta_{s}(\omega, y) d y .
$$

Notice that, for $P$-a.e. $\omega \in \Omega, f_{\varepsilon}(\omega, s, z)=0$ for all $z \notin \mathcal{U}_{\varepsilon}(\phi(\omega, K))$ and all $s \in[0, T]$. Here $\mathcal{U}_{\varepsilon}(\phi(\omega, K))$ is an $\varepsilon$-neighbor of $\phi(\omega, K)$ (assuming that the support of $\vartheta$ is in $B(1 / 2)$ ). And $f_{\varepsilon}(\omega, s, \cdot)$ is continuous. Hence $\int f_{\varepsilon}(s, z) \cdot D u_{s}(d z)$ is meaningful. We have

$$
\begin{gathered}
\left|\int_{0}^{t}\left(\int \mathcal{R}_{\varepsilon}\left[u_{s}, b_{s}\right](y) \theta_{s}(y) d y\right) d s\right| \leq \int_{0}^{t}\left(\int\left|f_{\varepsilon}(s, z)\right|\left|D u_{s}\right|(d z)\right) d s \\
\leq \int_{0}^{t}\left|D u_{s}\right|\left(\mathcal{U}_{1}(\phi(\omega, K))\right)\left\|f_{\varepsilon}(\omega, s, \cdot)\right\|_{L^{\infty}\left(\mathcal{U}_{1}(\phi(\omega, K))\right)} d s
\end{gathered}
$$

for all $\varepsilon \leq 1$. Moreover, with $C(\omega)=\| \theta$. $(\omega, \cdot) \|_{L^{\infty}([0, T] \times \phi(\omega, K))}$, we have

$$
\begin{aligned}
\left|f_{\varepsilon}(\omega, s, z)\right| & \leq C(\omega) \int_{\phi(\omega, K) \cap B(z, \varepsilon)} \vartheta_{\varepsilon}(y-z)\left|b_{s}(z)-b_{s}(y)\right| d y \\
& \leq C(\omega) \int_{B(1)} \vartheta(h)\left|b_{s}(z)-b_{s}(z+\varepsilon h)\right| d h \leq C^{\prime}(\omega) \varepsilon^{\alpha}
\end{aligned}
$$

uniformly in $(s, z)$. Hence

$$
\left|\int_{0}^{t}\left(\int \mathcal{R}_{\varepsilon}\left[u_{s}, b_{s}\right](y) \theta_{s}(y) d y\right) d s\right| \leq C^{\prime}(\omega) \varepsilon^{\alpha} \int_{0}^{T}\left|D u_{s}\right|\left(\mathcal{U}_{1}(\phi(\omega, K))\right) d s
$$

which converges to zero as $\varepsilon \rightarrow 0$, for $P$-a.s. $\omega \in \Omega$. The proof is complete.

\section{B The perturbative equation}

In this section we give a pathwise formulation of the SPDE, that we call perturbative equation. It does not involve stochastic integrals explicitly. We use it to prove Theorem 30; the absence of stochastic integrals simplify the analysis of some limits. Moreover, we think it may have other applications.

For streamlining the notations in this section we will let

$$
W_{t s}=W_{t}-W_{s}, \quad t \geq s \geq 0, \quad v_{t}(\theta)=\int_{\mathbb{R}^{d}} \theta(x) v(t, x) d x, \quad t \in[0, T],
$$

for any $v \in L_{\text {loc }}^{1}\left([0, T] \times \mathbb{R}^{d}\right), \quad \theta \in C_{0}^{\infty}\left(\mathbb{R}^{d}, \mathbb{R}^{k}\right)$. 
Theorem 26 Let $b \in L_{\text {loc }}^{1}\left([0, T] \times \mathbb{R}^{d} ; \mathbb{R}^{d}\right)$, $\operatorname{div} b \in L_{\text {loc }}^{1}\left([0, T] \times \mathbb{R}^{d}\right)$ and $u_{0} \in L^{\infty}\left(\mathbb{R}^{d}\right) . \quad A$ process $u \in L^{\infty}\left(\Omega \times[0, T] \times \mathbb{R}^{d}\right)$ is a weak $L^{\infty}$-solution of the SPDE (11) if and only if, for every $\theta \in C_{0}^{\infty}\left(\mathbb{R}^{d}\right), u_{t}(\theta)$ has a continuous adapted modification and the perturbative equation

$$
u_{t}(\theta)=u_{0}\left(\theta\left(\cdot+W_{t}\right)\right)+\int_{0}^{t} d s \int\left[b_{s}(x) \cdot D \theta\left(x+W_{t s}\right)+\operatorname{div} b_{s}(x) \theta\left(x+W_{t s}\right)\right] u_{s}(x) d x
$$

holds almost surely in $\omega \in \Omega$, for every $t \in[0, T]$.

Remark 27 Given $\theta \in C_{0}^{\infty}\left(\mathbb{R}^{d}\right)$ and $t \in[0, T]$, the integral on the right-hand-side of equation (31) is a well defined random variable, in spite of the only local integrability of $b$ and div $b$. Indeed, for P-a.e. $\omega \in \Omega, W .(\omega)$ is bounded on $[0, T]$ and thus $\theta\left(x+W_{t s}(\omega)\right)$ and $D \theta\left(x+W_{t s}(\omega)\right)$ vanish for $x$ outside a bounded set $B_{\omega} \subset \mathbb{R}^{d}$ ( $B_{\omega}$ depends on $\omega$ but not on $t$ and $s$ in $[0, T]$ ).

Proof. Step 1. Let us prove that a solution $u_{t}(\theta)$ of the perturbative equation is an $\mathcal{F}$ semimartingale and that the equation of Definition 12 is verified. We apply Itô formula to the process $F_{0}\left(W_{t}\right)$ and Itô-Wentzell-Kunita formula (see [23] ) to $F_{1}\left(t, W_{t}\right)$ where

$$
\begin{aligned}
F_{0}(y) & =u_{0}(\theta(\cdot+y)) \\
F_{1}(t, y) & =\int_{0}^{t} d s \int_{\mathbb{R}^{d}}\left[b_{s}(x) \cdot D \theta\left(x+y-W_{s}\right)+\operatorname{div} b_{s}(x) \theta\left(x+y-W_{s}\right)\right] u_{s}(x) d x
\end{aligned}
$$

$t \in[0, T], x \in \mathbb{R}^{d}$. Notice that $F_{1}(t, y, \omega)$, although being a random field, is of bounded variation (and more) in $t$, namely it has no martingale part, and

$$
\begin{aligned}
f_{1}(t, y) & =\frac{\partial F_{1}(t, y)}{\partial t} \\
& =\int_{\mathbb{R}^{d}}\left[b_{t}(x) \cdot D \theta\left(x+y-W_{t}\right)+\operatorname{div} b_{t}(x) \theta\left(x+y-W_{t}\right)\right] u_{t}(x) d x .
\end{aligned}
$$

Moreover $F_{1}(t, y, \omega)$ and $F_{0}(y)$ are smooth in $y$. Thus Itô-Wentzell-Kunita formula reduces to the classical Itô formula

$$
\begin{aligned}
d u_{t}(\theta) & =f_{1}\left(t, W_{t}\right) d t+\left(D F_{0}\left(W_{t}\right)+D F_{1}\left(t, W_{t}\right)\right) \cdot d W_{t} \\
& +\frac{1}{2}\left(\Delta F_{0}\left(W_{t}\right)+\Delta F_{1}\left(t, W_{t}\right)\right) d t .
\end{aligned}
$$

Notice that $D_{i} F_{0}\left(W_{t}\right)+D_{i} F_{1}\left(t, W_{t}\right)=u_{t}\left(D_{i} \theta\right)$ and $\Delta F_{0}\left(W_{t}\right)+\Delta F_{1}\left(t, W_{t}\right)=u_{t}(\Delta \theta)$. A substitution yields

$$
\begin{aligned}
d u_{t}(\theta) & =\int_{\mathbb{R}^{d}}\left[b_{t}(x) \cdot D \theta(x)+\operatorname{div} b_{t}(x) \theta(x)\right] u_{t}(x) d x d t \\
& +\sum_{i=1}^{d} u_{t}\left(D_{i} \theta\right) d W_{t}^{i}+\frac{1}{2} u_{t}(\Delta \theta) d t
\end{aligned}
$$

which shows that $u_{t}(\theta)$ is a semimartingale. For the same reason, also $u_{t}\left(D_{i} \theta\right)$ is a semimartingale, for each $i=1, \ldots, d$. Hence the Stratonovich integral $\int_{0}^{t} u_{s}\left(D_{i} \theta\right) \circ d W_{s}^{i}$ is well defined and is related to the Itô integral and to the joint quadratic variation by the formula (see [23])

$$
\int_{0}^{t} u_{s}\left(D_{i} \theta\right) \circ d W_{s}^{i}=\int_{0}^{t} u_{s}\left(D_{i} \theta\right) d W_{s}^{i}+\frac{1}{2}\left[M^{i}, W^{i}\right]_{t}
$$


where $M_{t}^{i}$ is the martingale part of $u_{t}\left(D_{i} \theta\right)$, which is equal to

$$
\sum_{j=1}^{d} \int_{0}^{t} u_{s}\left(D_{i j}^{2} \theta\right) d W_{s}^{j}
$$

(put $D_{i} \theta$ in place of $\theta$ in (44) above). Thus $\left[M^{i}, W^{i}\right]_{t}=\int_{0}^{t} u_{s}\left(D_{i} D_{i} \theta\right) d s$. Summarizing,

$$
\sum_{i=1}^{d} \int_{0}^{t} u_{s}\left(D_{i} \theta\right) \circ d W_{s}^{i}=\sum_{i=1}^{d} \int_{0}^{t} u_{s}\left(D_{i} \theta\right) d W_{s}^{i}+\frac{1}{2} \int_{0}^{t} u_{s}(\Delta \theta) d s .
$$

Together with equation (44), this proves that $u$ satisfies the equation of Definition 12 ,

Step 3. Let us now prove the converse statement. Let $u$ be an $L^{\infty}$-solution of the SPDE (1). Given $\theta \in C_{0}^{\infty}\left(\mathbb{R}^{d}\right)$ and $y \in \mathbb{R}^{d}$ let us take the test function $\theta_{y}(x)=\theta(x+y)$ in the weak formulation of the SPDE. We get

$$
\begin{aligned}
& d_{t} \int_{\mathbb{R}^{d}} u(t, x) \theta(x+y) d x \\
& =\int_{\mathbb{R}^{d}}[b(t, x) \cdot D \theta(x+y)+\operatorname{div} b(t, x) \theta(x+y)] u(t, x) d x d t \\
& +\sum_{i=1}^{d} \int_{\mathbb{R}^{d}} u(t, x) D_{i} \theta(x+y) d x \circ d W_{t}^{i} .
\end{aligned}
$$

Consider now the random field $\Theta(t, y)=\int_{\mathbb{R}^{d}} u(t, x) \theta(x+y) d x$. Given $t_{1} \in(0, T]$, we apply Itô-Wentzell-Kunita formula, in Stratonovich form, to $t \mapsto \Theta\left(t, y-W_{t}\right)$ for $t \in\left[0, t_{1}\right]$ (see [23]). We get

$$
\begin{gathered}
d \Theta\left(t, y-W_{t}\right)=\left.d \Theta(t, z)\right|_{z=y-W_{t}}-D \Theta\left(t, y-W_{t}\right) \circ d W_{t} \\
=\int_{\mathbb{R}^{d}}\left[b(t, x) \cdot D \theta\left(x+y-W_{t}\right)+\operatorname{div} b(t, x) \theta\left(x+y-W_{t}\right)\right] u(t, x) d x d t \\
+\sum_{i=1}^{d} \int_{\mathbb{R}^{d}} u(t, x) D_{i} \theta\left(x+y-W_{t}\right) d x \circ d W_{t}^{i} \\
-\sum_{i=1}^{d} \int_{\mathbb{R}^{d}} u(t, x) D_{i} \theta\left(x+y-W_{t}\right) d x \circ d W_{t}^{i}
\end{gathered}
$$

The last two terms coincide. Thus, integrating on $\left[0, t_{1}\right]$ we get

$$
\begin{aligned}
& \Theta\left(t_{1}, y-W_{t_{1}}\right)-\Theta(0, y) \\
& =\int_{0}^{t_{1}} \int_{\mathbb{R}^{d}}\left[b(s, x) \cdot D \theta\left(x+y-W_{s}\right)+\operatorname{div} b(s, x) \theta\left(x+y-W_{s}\right)\right] u(s, x) d x d s .
\end{aligned}
$$

All the terms are a.s. smooth functions of $y$, then taking $y=W_{t_{1}}$ and substituting the definition of $\Theta$ we get the perturbative equation. The proof is complete. 


\section{Wong-Zakai approximation results}

In this Appendix we prove Wong-Zakai results to motivate the Stratonovich integral in the SPDE (1). Since this is a side result for the purpose of this work, here we do not aim at full generality.

Wong-Zakai principle states that the solutions to equations where the noise is approximated by more regular processes converge to the solution of the stochastic differential equation with Stratonovich integrals. In contrast with the classical literature on the subject, here we meet a new difficulty: the approximating equations could miss uniqueness of solutions, since they are deterministic transport equations depending on a random parameter and the regularity of $b$ assumed in this work does not suffice for uniqueness. The most general statement of Wong-Zakai type, thus, would claim that all possible $L^{\infty}$ solutions of the approximating equations converge to the unique solution of the SPDE (we have proved uniqueness for the SPDE in the previous sections). However, for the deterministic transport equation, under our assumptions on $b$, there is no control on solutions, no representation in terms of a flow and it is not even clear how to prove bounds on them (in spite of the fact that, formally speaking, the $L^{\infty}$ norm should not increase in time).

Because of these difficulties, we restrict ourselves to two manageable situations. In the first case we regularize not only the noise but also the field $b$, so that the approximating equations are well posed. In the second one we consider a sequence of solutions of the approximating equations which fulfill a uniform bound (such sequences always exist under our hypotheses).

Let us mention that there exist several results of Wong-Zakai type for stochastic partial differential equations; let us quote only [16], [35] and [5] and references therein, as examples of results for parabolic and transport-type equations. Several works are based on stochastic characteristics and a Wong-Zakai result for them, which is also one of our strategies below. However, at the level of characteristics, all works assume sufficient regularity of coefficients to be able to make estimates of differences of solutions. Under our weak assumptions on $b$, we use a different approach, based on the compactness method. The strong well posedness of the limit equation is the key tool. Also the second theorem, not based on characteristics, is proved by a compactness argument.

Given a $d$-dimensional Brownian motion $W$ on a probability space $(\Omega, F, P)$, let $\left(W_{n}\right)_{n \geq 1}$, be a sequence of processes on the same space such that ( $T>0$ is given) $W_{n}$ converges in probability to $W$ in the topology of $C^{0}\left([0, T] ; \mathbb{R}^{d}\right)$.

An example is

$$
W_{n}(t)=\int_{0}^{\infty} n \theta(n(t-s)) W(s) d s
$$

where $\theta$ is a smooth non negative function with support in $(-1,1)$ and $\int_{-\infty}^{\infty} \theta(r) d r=1$.

Theorem 28 Assume that hypothesis 1 hold and $\operatorname{div} b \in L^{1}\left(0, T ; L_{\text {loc }}^{1}\left(\mathbb{R}^{d}\right)\right)$. Assume $u_{0} \in$ $C_{b}^{0}\left(\mathbb{R}^{d}\right)$. Let $\left\{b_{n}\right\}_{n \geq 1}$ be a sequence of equibounded (in $(t, x, n)$ ) measurable fields such that for a.e. $t \in[0, T]$ we have $b_{n}(t, \cdot) \in C_{b}^{1}\left(\mathbb{R}^{d}\right)$ and $b_{n}(t, \cdot) \rightarrow b(t, \cdot)$ uniformly on compact sets. Let $u_{n}(t, x)$ be the unique $L^{\infty}$ solution of the equation

$$
\frac{\partial u_{n}(t, x)}{\partial t}+b_{n}(t, x) \cdot D u_{n}(t, x)+\sum_{i=1}^{d} D_{i} u_{n}(t, x) \frac{d W_{n}^{i}(t)}{d t}=0, \quad u_{n}(0, x)=u_{0}(x)
$$

and let $u(t, x)=u_{0}\left(\varphi_{t}^{-1}(x)\right)$ be the solution of equation (11) given by Theorem 16. Then, for every $t \geq 0$ and $x \in \mathbb{R}^{d}, u_{n}(t, x)$ converges in probability to $u(t, x)$. 
Proof. We have $u_{n}(t, x)=u_{0}\left(\varphi_{n, t}^{-1}(x)\right)$ where $\varphi_{n, t}$ is the flow associated to the random equation

$$
\frac{d X_{n}(t)}{d t}=b_{n}\left(t, X_{n}(t)\right)+\frac{d W_{n}(t)}{d t} .
$$

Thus it is sufficient to prove that $\varphi_{n, t}^{-1}(x) \rightarrow \varphi_{t}^{-1}(x)$ in probability, given $t \geq 0$ and $x \in \mathbb{R}^{d}$.

The equations satisfied by the inverse flows are entirely similar to the equations for the direct flows. Thus, just for simplicity of notations, let us prove that $\varphi_{n, t}(x) \rightarrow \varphi_{t}(x)$ in probability, given $t \geq 0$ and $x \in \mathbb{R}^{d}$. For shortness, denote $\varphi_{n, t}(x)$ by $X_{n}(t)$ and $\varphi_{t}(x)$ by $X(t)$ (the initial condition $x$ is given).

Convergence in law would be classical. Thanks to the idea of [15], we can prove also convergence in probability, due to strong uniqueness for the limit equation. Let us recall some detail, similar to [15] (but here we have to deal also with the approximation of the noise).

Recall Lemma 1.1 from [15]. To prove the convergence in probability of $X_{n}(t)$ to $X(t)$ it is sufficient to prove the following property. Let $\left\{l_{k}\right\}_{k \geq 1}$ and $\left\{m_{k}\right\}_{k \geq 1}$ be two diverging sequences of natural numbers. We have to prove that there exist $\{k(j)\}_{j \geq 1}$ such that the pair $\left(X_{l_{k(j)}}(t), X_{m_{k(j)}}(t)\right)$ converges in law to a random element supported on the diagonal $\{(x, y) \in$ $\left.\mathbb{R}^{d} \times \mathbb{R}^{d}: x=y\right\}$.

We have

$$
X_{n}(t)=x+\int_{0}^{t} b_{n}\left(s, X_{n}(s)\right) d s+W_{n}(t)
$$

Since the sequence $\left\{b_{n}\right\}_{n \geq 1}$ is equibounded in all variables, the processes $\left\{\int_{0}^{\cdot} b_{n}\left(s, X_{n}(s)\right) d s\right\}_{n \geq 1}$ are equibounded in $C^{1}\left([0, T] ; \mathbb{R}^{d}\right)$. Then since $W_{n} \rightarrow W$ in $C\left([0, T], \mathbb{R}^{d}\right)$ in probability then the laws $\mu_{n}$ of $X_{n}$ on $C\left([0, T] ; \mathbb{R}^{d}\right)$ are tight, hence precompact by Prohorov theorem. We do not use explicitly this fact; we have described the argument in this simple case for later reference.

The argument now is similar to the proof of Theorem 2.4 of [15]. Given the two subsequences $\left\{l_{k}\right\}_{k \geq 1}$ and $\left\{m_{k}\right\}_{k \geq 1}$, let us repeat the previous argument for the process $Z_{k}=$ $\left(X_{l_{k}}, X_{m_{k}}, W_{l_{k}}, W_{m_{k}}\right)$. By Prohorov theorem there exist $\{k(j)\}_{j \geq 1}$ such that $Z_{k(j)}$ converges in law to a probability measure $\nu$ on $C\left([0, T] ; \mathbb{R}^{4 d}\right)$. By Skorokhod embedding theorem, there exists a new probability space $(\widetilde{\Omega}, \widetilde{F}, \widetilde{P})$ and random variables $\widetilde{Z}_{k(j)}=\left(\widetilde{X}_{l_{k(j)}}, \widetilde{X}_{m_{k(j)}}, \widetilde{W}_{l_{k(j)}}, \widetilde{W}_{m_{k(j)}}\right)$ with the same laws as $Z_{k(j)}$, and a random variable $\widetilde{Z}=\left(\widetilde{X}^{(1)}, \widetilde{X}^{(2)}, \widetilde{W}^{(1)}, \widetilde{W}^{(2)}\right)$ with law $\nu$, such that $\left\{\widetilde{Z}_{k(j)}\right\}_{j \geq 1}$ converges $\widetilde{P}$-a.s. to $\widetilde{Z}$ in the topology of $C\left([0, T] ; \mathbb{R}^{4 d}\right)$. It is easy to deduce that $\widetilde{W}^{(1)}$ and $\widetilde{W}^{(2)}$ are Brownian motions. Using the bounded continuous function $\varphi\left(\gamma^{(1)}, \gamma^{(2)}\right)=\frac{\left\|\gamma^{(1)}-\gamma^{(2)}\right\|_{0}}{1+\left\|\gamma^{(1)}-\gamma^{(2)}\right\|_{0}}$ on $C\left([0, T] ; \mathbb{R}^{d}\right)^{2}$, we see that

$$
\begin{aligned}
E\left[\varphi\left(\widetilde{W}^{(1)}, \widetilde{W}^{(2)}\right)\right] & =\lim _{j \rightarrow \infty} E\left[\varphi\left(\widetilde{W}_{l_{k(j)}}, \widetilde{W}_{m_{k(j)}}\right)\right] \\
& =\lim _{j \rightarrow \infty} E\left[\varphi\left(W_{l_{k(j)}}, W_{m_{k(j)}}\right)\right] \\
& =E[\varphi(W, W)]=0
\end{aligned}
$$

hence $\widetilde{W}^{(1)}=\widetilde{W}^{(2)}$. With a similar argument, one can check that $\widetilde{X}_{l_{k(j)}}, \widetilde{W}_{l_{k(j)}}$ are related by the equation

$$
\widetilde{X}_{l_{k(j)}}(t)=x+\int_{0}^{t} b_{l_{k(j)}}\left(s, \widetilde{X}_{l_{k(j)}}(s)\right) d s+\widetilde{W}_{l_{k(j)}}(t)
$$


and similarly for the pair $\widetilde{X}_{m_{k(j)}}, \widetilde{W}_{m_{k(j)}}$. From the $\widetilde{P}$-a.s. convergence in $C\left([0, T] ; \mathbb{R}^{d}\right)$ of all processes, the uniform convergence of $b_{l_{k(j)}}(s, \cdot)$ to $b(s, \cdot)$ on compact sets (a.s. in $s$ ), and the equiboundedness of $b_{l_{k(j)}}$, by Lebesgue dominated convergence theorem we get

$$
\widetilde{X}^{(i)}(t)=x+\int_{0}^{t} b\left(s, \widetilde{X}^{(i)}(s)\right) d s+\widetilde{W}(t)
$$

for $i=1,2$, where $\widetilde{W}=\widetilde{W}^{(1)}=\widetilde{W}^{(2)}$. By strong uniqueness for this equation we deduce $\widetilde{X}^{(1)}=\widetilde{X}^{(2)}$. Hence $\left(\widetilde{X}_{l_{k(j)}}(t), \widetilde{X}_{m_{k(j)}}(t)\right)$ converges in law to a random element supported on the diagonal $\left\{(x, y) \in \mathbb{R}^{d} \times \mathbb{R}^{d}: x=y\right\}$. This implies the claim. The proof is complete.

Remark 29 A more difficult form of Wong-Zakai result would be to prove that any sequence $\left\{u_{n}\right\}$ of $L^{\infty}$ solutions of the (a priori) not well posed equations

$$
\frac{\partial u_{n}}{\partial t}+b \cdot D u_{n}+\sum_{i=1}^{d} D_{i} u_{n} \cdot \frac{d W_{n}^{i}}{d t}=0, \quad u^{n}(0, x)=u_{0}(x)
$$

converges to the unique solution $u$ of the SPDE (here we do not regularize b). Under our assumptions on $b$, it is very difficult to deal with $L^{\infty}$ solutions of this 'deterministic' equation. For instance, if we want to perform computations (for proving estimates, comparisons, etc.), we have to regularize the solution and control the behavior of a commutator, which is an open problem under this regularity of $b$ and $u^{n}$ (this problem is the same as proving that weak solutions are renormalizable, in the sense of DiPerna-Lions [10], problem solved under other conditions on b).

As a partial result towards a general convergence statement we propose the following theorem dealing with convergence of a particular class of non-unique solutions to the transport equation.

Theorem 30 Let $b \in L_{\mathrm{loc}}^{1}\left([0, T] \times \mathbb{R}^{d} ; \mathbb{R}^{d}\right)$, div $b \in L_{\mathrm{loc}}^{1}\left([0, T] \times \mathbb{R}^{d}\right)$ and $u_{0} \in L^{\infty}\left(\mathbb{R}^{d}\right)$. Let $u^{n}$ be a sequence of $L^{\infty}\left(\Omega \times[0, T] \times \mathbb{R}^{d}\right)$ functions which are weak solutions of the PDEs

$$
\partial_{t} u_{t}^{n}+b_{t} \cdot D u_{t}^{n}+\sum_{i=1}^{d} D_{i} u_{t}^{n} \partial_{t} W_{n}^{i}(t)=0
$$

with the same initial condition $u_{0}$. Assume the following conditions

(i) $\mathcal{F}_{t}^{n}=\sigma\left(W_{n}(s): s \leq t\right)$ converge to $\mathcal{F}_{t}=\sigma\left(W_{s}: s \leq t\right)$ as $n \rightarrow \infty$ for any $t$ in the sense that $\mathbb{E}\left[F \mid \mathcal{F}_{t}^{n}\right] \rightarrow \mathbb{E}\left[F \mid \mathcal{F}_{t}\right]$ almost surely for any bounded r.v. $F$.

(ii) The family $\left\{u^{n}\right\}$ is equibounded in $L^{\infty}\left(\Omega \times[0, T] \times \mathbb{R}^{d}\right)$ and $u^{n}$ is $\mathcal{F}^{n}$-progressively measurable for any $n$.

Then up to extraction of a subsequence still denoted by $u^{n}$ we have weak-* convergence to $u \in L^{\infty}\left(\Omega \times[0, T] \times \mathbb{R}^{d}\right)$ satisfying the SPDE P-almost surely. Moreover under Hypotheses 1$]$ and 2 the whole sequence converge to the unique $L^{\infty}$-solution of the SPDE. 
Note that by regularization and by compactness it is not difficult to show that the equibounded family of solutions $\left\{u^{n}\right\}_{n \geq 1}$ of the equations (46) exists (condition (ii)). Moreover the convergence of the conditional expectations (condition (i)) holds for our example 45] since the mollifier is of compact support (in that case $\mathcal{F}_{t}^{n} \subseteq \mathcal{F}_{t+c / n}$ for some $c>0$ and the filtration $\mathcal{F}$ is continuous). Unfortunately, as we already stressed, we are not able to deal with an arbitrary family of solutions to the approximating problems (46).

Proof. By an adaptation of Theorem [26 the $u^{n}$ are shown to satisfy the perturbative equation

$$
\begin{gathered}
u_{t}^{n}(\theta)=u_{0}\left(\theta\left(\cdot+W_{n}(t)\right)\right) \\
+\int_{0}^{t}\left[b(s, x) \cdot D \theta\left(x+W_{n}(t)-W_{n}(s)\right)+\operatorname{div} b(s, x) \theta\left(x+W_{n}(t)-W_{n}(s)\right)\right] u_{s}^{n}(x) d x d s
\end{gathered}
$$

for any $\theta \in C_{0}^{\infty}\left(\mathbb{R}^{d}\right)$. By equiboundedness in $L^{\infty}$ we can pass to a weakly-* convergent subsequence (still called $u^{n}$ ) and we have that the weak-* limit $u$ is in $L^{\infty}$ and satisfies

$$
u_{t}(\theta)=u_{0}\left(\theta\left(\cdot+W_{t}\right)\right)+\int_{0}^{t}\left[b(s, x) \cdot D \theta\left(x+W_{t s}\right)+\operatorname{div} b(s, x) \theta\left(x+W_{t s}\right)\right] u_{s}(x) d x d s
$$

for a.e. $(\omega, t) \in \Omega \times[0, T]$. The right-hand-side of this equation is a well defined random variable, for every given $\theta \in C_{0}^{\infty}\left(\mathbb{R}^{d}\right.$ ) and $t \in[0, T]$ (see remark 27). With little more work one can see that, as a stochastic process in $t$, it is $P$-a.s. continuous. Let us prove this in detail for completeness. We give the details only for the double integral, that we denote by $I_{t}(\omega)$. Take a representative $\widetilde{u}$ of the equivalence class of $u$, that is a bounded measurable function. For all $\omega$ in a full measure set $\Omega_{1}, \widetilde{u}$ is a bounded measurable function of $(t, x)$. Let $\Omega_{2}$ be a full measure set where $W$ is continuous on $[0, T]$. For every $\omega$ in the full measure set $\Omega_{1} \cap \Omega_{2}$ we have two integrals of the form

$$
\int_{0}^{t} \int_{\mathbb{R}^{d}} a(s, x) \varphi\left(x+W_{t s}(\omega)\right) d x d s
$$

where $a$ is deterministic and integrable, $\varphi \in C_{0}^{\infty}\left(\mathbb{R}^{d}\right)$ and $W_{t s}(\omega)$ is continuous in $t$ and $s$. Then this integral is continuous in $t$. This proves that the double integral is a continuous process.

By Theorem 26, $u$ satisfies the SPDE as soon as we can prove that the continuous process $u_{t}(\theta)$ is adapted. We have that $u_{t}^{n}(\theta)$ converges to $u_{t}(\theta)$ weak-* in $L^{\infty}(\Omega \times[0, T])$, hence $\mathbb{E}\left[\int_{0}^{T} u_{t}^{n}(\theta) f_{t} G d t\right]$ converges to $\mathbb{E}\left[\int_{0}^{T} u_{t}(\theta) f_{t} G d t\right]$ for every bounded r.v. $G$ and bounded measurable function $f:[0, T] \rightarrow \mathbb{R}$. Moreover, $u_{t}^{n}(\theta)$ is $\mathcal{F}_{t}^{n}$-measurable. Then for any bounded r.v. $G$ we have $\mathbb{E}\left[u_{t}^{n}(\theta) G\right]=\mathbb{E}\left[u_{t}^{n}(\theta) \mathbb{E}\left[G \mid \mathcal{F}_{t}^{n}\right]\right]$. Therefore $\int_{0}^{T} f_{t} \mathbb{E}\left[u_{t}^{n}(\theta) \mathbb{E}\left[G \mid \mathcal{F}_{t}^{n}\right]\right] d t$ converges to $\mathbb{E}\left[\int_{0}^{T} u_{t}(\theta) f_{t} G d t\right]$.

Moreover, we can write

$$
\mathbb{E}\left[u_{t}^{n}(\theta) \mathbb{E}\left[G \mid \mathcal{F}_{t}^{n}\right]\right]=\mathbb{E}\left[u_{t}^{n}(\theta) \mathbb{E}\left[G \mid \mathcal{F}_{t}\right]\right]+\mathbb{E}\left[u_{t}^{n}(\theta)\left(\mathbb{E}\left[G \mid \mathcal{F}_{t}^{n}\right]-\mathbb{E}\left[G \mid \mathcal{F}_{t}\right]\right)\right]
$$

and thus, by the a.s. convergence of $\mathbb{E}\left[G \mid \mathcal{F}_{t}^{n}\right] \rightarrow \mathbb{E}\left[G \mid \mathcal{F}_{t}\right]$ together with the dominated convergence theorem we get that $\int_{0}^{T} f_{t} \mathbb{E}\left[u_{t}^{n}(\theta) \mathbb{E}\left[G \mid \mathcal{F}_{t}\right]\right] d t$ converges to $\mathbb{E}\left[\int_{0}^{T} u_{t}(\theta) f_{t} G d t\right]$. But the quantity $\int_{0}^{T} f_{t} \mathbb{E}\left[u_{t}^{n}(\theta) \mathbb{E}\left[G \mid \mathcal{F}_{t}\right]\right] d t$ converges also to $\int_{0}^{T} f_{t} \mathbb{E}\left[u_{t}(\theta) \mathbb{E}\left[G \mid \mathcal{F}_{t}\right]\right] d t$.

We thus obtain that $\mathbb{E}\left[u_{t}(\theta) G\right]=\mathbb{E}\left[u_{t}(\theta) \mathbb{E}\left[G \mid \mathcal{F}_{t}\right]\right]$ for any bounded r.v. $G$ showing that $u_{t}(\theta)$ is $\mathcal{F}_{t}$-measurable. The proof of the first claim of the theorem is complete.

If Hypotheses 1 and 2 hold, each weak-* convergent subsequence converge to the unique solution of the SPDE so that the extraction of a subsequence is not necessary. 


\section{Two additional uniqueness results in $L^{\infty}$}

The aim of this section is to prove some complementary uniqueness results for $L^{\infty}$ weak solutions of the SPDE obtained extending the key estimates in fractional Sobolev spaces. The first result is the following:

Theorem 31 Let $d \geq 2$. Assume Hypothesis 1 and also that $\operatorname{div} b \in L^{q}\left(0, T ; L^{p}\left(\mathbb{R}^{d}\right)\right)$ for some $q>2 \geq p>\frac{2 d}{d+2 \alpha}$. Then there exists a unique weak $L^{\infty}$-solution $u$ of the Cauchy problem (1) and $u(t, x)=u_{0}\left(\phi_{t}^{-1}(x)\right)$.

The main interest of this result is due to the fact that we can consider some $p$ in the critical interval $(1,2]$ not covered by Hypothesis 2 .

Another result deals with an additional hypothesis of Sobolev regularity for $b$ (beside the usual Hölder regularity) which allow to relax the hypothesis on $\operatorname{div} b$.

Theorem 32 Assume that $\operatorname{div} b \in L_{\text {loc }}^{1}\left([0, T] \times \mathbb{R}^{d}\right)$ and that

$$
b \in L^{1}\left(0, T ; W_{\text {loc }}^{\theta, 1}\left(\mathbb{R}^{d}\right)\right) \cap L^{\infty}\left(0, T ; C^{\alpha}\left(\mathbb{R}^{d}\right)\right)
$$

with $\alpha>0, \theta>0$ and $\alpha+\theta>1$. Then there exists a unique weak $L^{\infty}$-solution $u$ of the Cauchy problem (1) and $u(t, x)=u_{0}\left(\phi_{t}^{-1}(x)\right)$.

The proofs of both theorems follow the proof of Theorem 21 using the results below on the commutator and on the regularity of the Jacobian of the flow. Since these results are complementary the details of the proofs are left to the reader. The following commutator estimates follows from Lemma 22 ,

Corollary 33 Assume $v \in L_{l o c}^{\infty}\left(\mathbb{R}^{d}, \mathbb{R}^{d}\right)$, div $v \in L_{l o c}^{1}\left(\mathbb{R}^{d}\right), g \in L_{l o c}^{\infty}\left(\mathbb{R}^{d}\right)$.

(i) If there exists $\theta \in(0,1)$ such that $v \in W_{\mathrm{loc}}^{\theta, 1}\left(\mathbb{R}^{d}, \mathbb{R}^{d}\right)$, then

$$
\left|\int \mathcal{R}_{\varepsilon}[g, v](x) \rho(x) d x\right| \leq C_{r}\|g\|_{L_{r+1}^{\infty}}\left(\|\rho\|_{L_{r}^{\infty}}\|\operatorname{div} v\|_{L_{r+1}^{1}}+[\rho]_{C_{r}^{1-\theta}}[v]_{W_{r+1}^{\theta, 1}}\right) .
$$

(ii) If there exists $\alpha \in(0,1)$ such that $v \in C_{\mathrm{loc}}^{\alpha}\left(\mathbb{R}^{d}, \mathbb{R}^{d}\right)$, then

$$
\left|\int \mathcal{R}_{\varepsilon}[g, v](x) \rho(x) d x\right| \leq C_{r}\|g\|_{L_{r+1}^{\infty}}\left(\|\rho\|_{L_{r}^{\infty}}\|\operatorname{div} v\|_{L_{r+1}^{1}}+[v]_{C_{r+1}^{\alpha}}[\rho]_{W_{r}^{1-\alpha, 1}}\right) .
$$

Proof. We have

$$
\begin{gathered}
\left|\iint g\left(x^{\prime}\right) D_{x} \vartheta_{\varepsilon}\left(x-x^{\prime}\right)\left(\rho(x)-\rho\left(x^{\prime}\right)\right)\left[v(x)-v\left(x^{\prime}\right)\right] d x d x^{\prime}\right| \\
\leq \frac{\varepsilon^{1-\theta}}{\varepsilon}[\rho]_{C_{r}^{1-\theta}}\|g\|_{L_{r+1}^{\infty}} \frac{1}{\varepsilon^{d}} \iint_{B(r+1)^{2}}\left|D_{x} \vartheta\left(\frac{x-x^{\prime}}{\varepsilon}\right)\right| \frac{\left|v(x)-v\left(x^{\prime}\right)\right|}{\left|x-x^{\prime}\right|^{\theta+d}}\left|x-x^{\prime}\right|^{\theta+d} d x d x^{\prime} \\
\leq[\rho]_{C_{r}^{1-\theta}}\|g\|_{L_{r+1}^{\infty}}\|D \theta\|_{\infty}[v]_{W_{r+1}^{\theta, 1}}
\end{gathered}
$$

The second statement has a similar proof.

These results can be extended to the case in which commutators are composed with a flow. 
Lemma 34 Let $\phi$ be a $C^{1}$-diffeomorphism of $\mathbb{R}^{d}$ (J申 denotes its Jacobian). Assume $v \in$ $L_{l o c}^{\infty}\left(\mathbb{R}^{d}, \mathbb{R}^{d}\right), \operatorname{div} v \in L_{l o c}^{1}\left(\mathbb{R}^{d}\right), g \in L_{l o c}^{\infty}\left(\mathbb{R}^{d}\right)$.

Then, for any $\rho \in C_{r}^{\infty}\left(\mathbb{R}^{d}\right)$ and any $R>0$ such that $\operatorname{supp}\left(\rho \circ \phi^{-1}\right) \subseteq B(R)$, we have a uniform bound of $\int \mathcal{R}_{\varepsilon}[g, v](\phi(x)) \rho(x) d x$ under one of the following conditions:

(i) there exists $\theta \in(0,1)$ such that $v \in W_{\text {loc }}^{\theta, 1}\left(\mathbb{R}^{d}, \mathbb{R}^{d}\right), J \phi \in C_{\text {loc }}^{1-\theta}\left(\mathbb{R}^{d}\right)$;

(ii) there exists $\alpha \in(0,1)$ such that $J \phi \in W_{\mathrm{loc}}^{1-\alpha, 1}\left(\mathbb{R}^{d}\right), v \in C_{\mathrm{loc}}^{\alpha}\left(\mathbb{R}^{d}, \mathbb{R}^{d}\right)$.

Moreover, under one of the previous conditions, we also have

$$
\lim _{\varepsilon \rightarrow 0} \int \mathcal{R}_{\varepsilon}[g, v](\phi(x)) \rho(x) d x=0 .
$$

Proof. By a change of variables $\int \mathcal{R}_{\varepsilon}[g, v](\phi(x)) \rho(x) d x=\int \mathcal{R}_{\varepsilon}[g, v](y) \rho_{\phi}(y) d x$ where the function $\rho_{\phi}(y)=\rho\left(\phi^{-1}(y)\right) J \phi^{-1}(y)$ has the support strictly contained in the ball of radius $R$. Clearly, $\left\|\rho_{\phi}\right\|_{L_{R}^{\infty}} \leq\|\rho\|_{L_{r}^{\infty}}\left\|J \phi^{-1}\right\|_{L_{R}^{\infty}}$. To prove the result, we have to check that Corollary 33 can be applied with $\rho_{\phi}$ instead of $\rho$.

(i) To apply Corollary 33 (i), we need to check that $\rho_{\phi} \in C_{l o c}^{1-\theta}$. This follows since

$$
\begin{gathered}
{\left[\rho_{\phi}\right]_{C_{R}^{1-\theta}} \leq\left\|J \phi^{-1}\right\|_{L_{R}^{\infty}}\left[\rho\left(\phi^{-1}(\cdot)\right)\right]_{C_{R}^{1-\theta}}+\|\rho\|_{L_{r}^{\infty}}\left[J \phi^{-1}\right]_{C_{R}^{1-\theta}}} \\
\leq\left\|D \phi^{-1}\right\|_{L_{R}^{\infty}}\|D \rho\|_{L_{r}^{\infty}}\left[D \phi^{-1}\right]_{C_{R}^{1-\theta}}+\|\rho\|_{L_{r}^{\infty}}\left[D \phi^{-1}\right]_{C_{R}^{1-\theta}} .
\end{gathered}
$$

and the bound follows.

(ii) To apply Corollary 33 (ii), we need to check that $\rho_{\phi} \in W_{l o c}^{1-\alpha, 1}$ : first

$$
\left[\rho_{\phi}\right]_{W_{R}^{1-\alpha, 1}} \leq\left\|J \phi^{-1}\right\|_{L_{R}^{\infty}}\left[\rho \circ \phi^{-1}\right]_{W_{R}^{1-\alpha, 1}}+\left[J \phi^{-1}\right]_{W_{R}^{1-\alpha, 1}}\|\rho\|_{L_{r}^{\infty}}
$$

and since

$$
\left[\rho \circ \phi^{-1}\right]_{W_{R}^{1-\alpha, 1}} \leq\left\|D\left(\rho \circ \phi^{-1}\right)\right\|_{L_{R}^{1}} \leq\|D \rho\|_{L_{r}^{1}}\left\|D \phi^{-1}\right\|_{L_{R}^{\infty}}
$$

we find

$$
\left[\rho_{\phi}\right]_{W_{R}^{1-\alpha, 1}} \leq C_{R}\|D \rho\|_{L_{r}^{1}}\left\|D \phi^{-1}\right\|_{L_{R}^{\infty}}\left\|J \phi^{-1}\right\|_{L_{R}^{\infty}}+\left[J \phi^{-1}\right]_{W_{R}^{1-\alpha, 1}}\|\rho\|_{L_{r}^{\infty}}
$$

and the bound follows.

Finally the next theorem extends the analysis of the Jacobian of the flow presented in Section 3 and links the regularity condition on $J \phi$ required in Lemma 34 (ii) to the assumption on the divergence of $b$ stated in Theorem 31 .

Theorem 35 Let $d \geq 2$. Assume Hypothesis 1 and the existence of $p \in\left(\frac{2 d}{d+2 \alpha}, 2\right]$ and $q>2$ such that $\operatorname{div} b \in L^{q}\left(0, T ; L^{p}\left(\mathbb{R}^{d}\right)\right)$. Then, for any $r>0, J \phi \in L^{p}\left(0, T ; W_{r}^{1-\alpha, p}\right)$, P-a.s.

Proof. The first part of the proof is similar to the one of Theorem 11. Indeed Step 1 can be carried on thanks to the chain rule for fractional Sobolev spaces: if $f: \mathbb{R}^{d} \rightarrow \mathbb{R}$ is a continuous function, of class $W_{l o c}^{1-\alpha, p}\left(\mathbb{R}^{d}\right)$ and $g: \mathbb{R} \rightarrow \mathbb{R}$ is a $C^{\infty}$ function, then $g \circ f \in W_{l o c}^{1-\alpha, p}\left(\mathbb{R}^{d}\right)$ and

$$
[(g \circ f)]_{W_{r}^{1-\alpha, p}}^{p} \leq\left(\sup _{x \in B(r)}\left|g^{\prime}(f(x))\right|\right)^{p}[f]_{W_{r}^{1-\alpha, p}}^{p},
$$


for every $r>0$. The modification of Step 2 does not pose any problem, so we only consider the last steps of the proof.

Step 3. To prove the assertion it is enough to check that the family $\left(\psi_{\varepsilon}\right)_{\varepsilon>0}$ is bounded in $L^{p}\left(\Omega \times(0, T) ; W_{r}^{1-\alpha, p}\right)$.

Indeed, once we have proved this fact, we can extract from the previous sequence $\psi_{\varepsilon_{n}}$ a subsequence which converges weakly in $L^{p}\left(\Omega \times(0, T) ; W_{r}^{1-\alpha, p}\right)$ to some $\gamma$. This in particular implies that such subsequence converges weakly in $L^{p}\left(\Omega \times(0, T), L_{r}^{p}\right)$ to $\gamma$ so we must have that $\gamma=J \phi$.

We introduce the following Cauchy problem, for $\varepsilon \geq 0$,

$$
\left\{\begin{array}{rr}
\frac{\partial F^{\varepsilon}}{\partial t}+\frac{1}{2} \Delta F^{\varepsilon}+D F^{\varepsilon} \cdot b^{\varepsilon}=\operatorname{div} b^{\varepsilon}, & t \in[0, T[ \\
F^{\varepsilon}(T, x)=0, & x \in \mathbb{R}^{d} .
\end{array}\right.
$$

This problem has a unique solution $F^{\varepsilon}$ in the space $L^{q}\left(0, T ; W^{2, p}\left(\mathbb{R}^{d}\right)\right.$. Moreover, there exists a positive constant $C=C\left(p, q, d, T,\|b\|_{\infty}\right)$ such that

$$
\left\|F^{\varepsilon}\right\|_{L^{q}\left(0, T ; W^{2, p}\left(\mathbb{R}^{d}\right)\right)} \leq C\|\operatorname{div} b\|_{L^{q}\left(0, T ; L^{p}\left(\mathbb{R}^{d}\right)\right)},
$$

for any $\varepsilon \geq 0$. This result can be proved by using [18, Theorem 1.2] and repeating the argument of the proof in [21, Theorem 10.3]. This argument works without difficulties in the present case in which $b$ (and so $b^{\varepsilon}$ ) is globally bounded and $\operatorname{div} b \in L^{q}\left(0, T ; L^{p}\left(\mathbb{R}^{d}\right)\right)$ with $p, q \in(1,+\infty)$.

From the previous result we can also deduce, since we are assuming $q>2$, that $F^{\varepsilon} \in$ $C\left([0, T] ; W^{1, p}\left(\mathbb{R}^{d}\right)\right)$, for any $\varepsilon \geq 0$, and moreover there exists a positive constant $C=C(p, q$ $\left.d, T,\|b\|_{\infty}\right)$ such that

$$
\sup _{t \in[0, T]}\left\|F^{\varepsilon}(t, \cdot)\right\|_{W^{1, p}\left(\mathbb{R}^{d}\right)} \leq C\|\operatorname{div} b\|_{L^{q}\left(0, T ; L^{p}\left(\mathbb{R}^{d}\right)\right)} .
$$

We only give a sketch of proof of (49). Define $u^{\varepsilon}(t, x)=F^{\varepsilon}(T-t, x)$; we have the explicit formula

$$
u^{\varepsilon}(t, x)=\int_{0}^{t} P_{t-s} g^{\varepsilon}(s, \cdot)(x) d s
$$

where $\left(P_{t}\right)$ is the heat semigroup and $g^{\varepsilon}(t, x)=D u^{\varepsilon}(t, x) \cdot b^{\varepsilon}(T-t, x)-\operatorname{div} b^{\varepsilon}(T-t, x)$. We get, since $q>2$ and $q^{\prime}=\frac{q}{q-1}<2$,

$$
\left\|D_{x} u^{\varepsilon}(t, \cdot)\right\|_{L^{p}} \leq c \int_{0}^{t} \frac{1}{(t-s)^{1 / 2}}\left\|g^{\varepsilon}(s, \cdot)\right\|_{L^{p}} d s \leq C\left(\int_{0}^{T} \frac{1}{s^{q^{\prime} / 2}} d s\right)^{1 / q^{\prime}}\left(\int_{0}^{T}\|\operatorname{div} b(s, \cdot)\|_{L^{p}}^{q} d s\right)^{1 / q}
$$

and so (49) holds.

Using Itô formula we find $\left(\right.$ remark that $F^{\varepsilon}(t, \cdot) \in C_{b}^{2}\left(\mathbb{R}^{d}\right)$ )

$$
F^{\varepsilon}\left(t, \phi_{t}^{\varepsilon}(x)\right)-F^{\varepsilon}(0, x)-\int_{0}^{t} D F^{\varepsilon}\left(s, \phi_{s}^{\varepsilon}(x)\right) \cdot d W_{s} \int_{0}^{t} \operatorname{div} b^{\varepsilon}\left(s, \phi_{s}^{\varepsilon}(x)\right) d s=\psi_{\varepsilon}(t, x) .
$$

Since we already know that $\left(\psi_{\varepsilon}\right)_{\varepsilon>0}$ is bounded in $L^{p}\left(\Omega \times(0, T), L_{r}^{p}\right)$ and since $p \leq 2$, to verify that $\left(\psi_{\varepsilon}\right)_{\varepsilon>0}$ is bounded in $L^{p}\left(\Omega \times(0, T) ; W_{r}^{1-\alpha, p}\right)$, it is enough to prove that $E \int_{0}^{T}\left[\psi_{\varepsilon}(t, \cdot)\right]_{W_{r}^{1-\alpha, 2}}^{2} d t \leq$ 
$C$, for any $\varepsilon>0$. We give details only for the most difficult term $\int_{0}^{t} D F^{\varepsilon}\left(s, \phi_{s}^{\varepsilon}(x)\right) d W_{s}$ in (50). The $F(0, x)$ term can be controlled using (49) and the others are of easier estimation. We show that there exists a constant $C>0$ (independent on $\varepsilon$ ) such that

$$
E \int_{0}^{T} d t\left[\int_{0}^{t} D F^{\varepsilon}\left(s, \phi_{s}^{\varepsilon}(\cdot)\right) d W_{s}\right]_{W_{r}^{1-\alpha, 2}}^{2} \leq C
$$

We have

$$
\begin{gathered}
E\left[\int_{0}^{T} d t \int_{B(r)} \int_{B(r)} \frac{\left|\int_{0}^{t}\left(D F^{\varepsilon}\left(s, \phi_{s}^{\epsilon}(x)\right)-D F^{\varepsilon}\left(s, \phi_{s}^{\epsilon}\left(x^{\prime}\right)\right)\right) d W_{s}\right|^{2}}{\left|x-x^{\prime}\right|^{(1-\alpha) 2+d}} d x d x^{\prime}\right] \\
=\int_{0}^{T} \int_{B(r)} \int_{B(r)} E \int_{0}^{t} \frac{\left|D F^{\varepsilon}\left(s, \phi_{s}^{\epsilon}(x)\right)-D F^{\varepsilon}\left(s, \phi_{s}^{\epsilon}\left(x^{\prime}\right)\right)\right|^{2}}{\left|x-x^{\prime}\right|^{(1-\alpha) 2+d}} d s d x d x^{\prime}, \\
=E \int_{0}^{T} d t \int_{0}^{t} d s \int_{B(r)} \int_{B(r)} \frac{\left|D F^{\varepsilon}\left(s, \phi_{s}^{\epsilon}(x)\right)-D F^{\varepsilon}\left(s, \phi_{s}^{\epsilon}\left(x^{\prime}\right)\right)\right|^{2}}{\left|x-x^{\prime}\right|^{(1-\alpha) 2+d}} d x d x^{\prime} \\
\leq T E\left[\int_{0}^{T} d s \int_{B(r)} \int_{B(r)} \frac{\left|D F^{\varepsilon}\left(s, \phi_{s}^{\epsilon}(x)\right)-D F^{\varepsilon}\left(s, \phi_{s}^{\epsilon}\left(x^{\prime}\right)\right)\right|^{2}}{\left|x-x^{\prime}\right|^{(1-\alpha) 2+d}} d x d x^{\prime}\right], \\
\leq T E \int_{0}^{T}\left[D F^{\varepsilon}\left(s, \phi_{s}^{\epsilon}(\cdot)\right)\right]_{W_{r}^{1-\alpha, 2}}^{2} d s
\end{gathered}
$$

By the Sobolev embedding the $W_{r}^{1-\alpha, 2}$-seminorm can be controlled by the norm in $W_{r}^{1, p}$ if

$$
1-\frac{d}{p} \geq(1-\alpha)-\frac{d}{2}
$$

This means if $p \geq \frac{2 d}{d+2 \alpha}$. Then we consider $p_{1}$ such that $p>p_{1}>\frac{2 d}{d+2 \alpha}$ and show that

$$
E \int_{0}^{T}\left\|D F^{\varepsilon}\left(s, \phi_{s}^{\epsilon}(\cdot)\right)\right\|_{W_{r}^{1, p_{1}}}^{2} d s \leq C<\infty
$$

where $C$ is independent on $\varepsilon$.

Step 4. To obtain (52) we estimate

$$
E \int_{0}^{T} d s\left(\int_{B(r)}\left|D^{2} F^{\varepsilon}\left(s, \phi_{s}^{\epsilon}(x)\right) D \phi_{s}^{\epsilon}(x)\right|^{p_{1}} d x\right)^{\frac{2}{p_{1}}}
$$

A similar term has been already estimated in the proof of Theorem 11. Since

$$
\int_{B(r)}\left(\int_{0}^{T} E\left[\left|D \phi_{s}^{\epsilon}(x)\right|^{r}\right] d s\right)^{\gamma} d x<\infty
$$

for every $r, \gamma \geq 1$ (see (16)), by the Hölder inequality, it is sufficient to prove that

$$
\int_{0}^{T} E\left[\left(\int_{B(r)}\left|D^{2} F^{\varepsilon}\left(s, \phi_{s}^{\epsilon}(x)\right)\right|^{p} d x\right)^{\frac{2}{p}}\right] d t \leq C<\infty .
$$


We have

$$
\begin{aligned}
& \int_{0}^{T} E\left[\left(\int_{B(r)}\left|D^{2} F^{\varepsilon}\left(s, \phi_{s}^{\epsilon}(x)\right)\right|^{p} d x\right)^{\frac{2}{p}}\right] d t \\
& =E\left[\int_{0}^{T} d s\left(\int_{\phi^{\epsilon}{ }_{s}(B(r))}\left|D^{2} F^{\varepsilon}(s, y)\right|^{p} J\left(\phi^{\epsilon}{ }_{s}\right)^{-1}(y) d y\right)^{\frac{2}{p}}\right] \\
& \leq \sup _{s \in[0, T], y \in \mathbb{R}^{d}} E\left[J\left(\phi_{s}^{\varepsilon}\right)^{-1}(y)\right]^{2 / p} \int_{0}^{T}\left(\int_{\mathbb{R}^{d}}\left|D^{2} F^{\varepsilon}(s, y)\right|^{p} d y\right)^{\frac{2}{p}} \leq C<\infty,
\end{aligned}
$$

where, using the results of Section 2 and the bound (48), $C$ is independent on $\varepsilon>0$. The proof is complete.

\section{References}

[1] Albeverio S., Flandoli F., Sinai Y. G., SPDE in hydrodynamic: recent progress and prospects, Edited by G. Da Prato and M. Röckner, LNM 1942, Springer-Verlag, Berlin; Fondazione C.I.M.E., Florence, 2008.

[2] Ambrosio, L.: Transport equation and Cauchy problem for $B V$ vector fields, Invent. Math. 158, 227-260 (2004).

[3] Ambrosio, L., Crippa G.: Existence, uniqueness, stability and differentiability properties of the flow associated to weakly differentiable vector fields, in Transport Equations and Multi-D Hyperbolic Conservation Laws, Lecture Notes of the Unione Matematica Italiana 5, Springer Verlag, 2008.

[4] Brandt A.: Interior Schauder estimates for parabolic differential (or difference) equations via the maximum principle, Israel J. Math. 7 254-262 (1969).

[5] Brzezniak Z., Flandoli F.: Almost sure approximation of Wong-Zakai type for stochastic partial differential equations, Stochastic Process. Appl. 55, 329-358 (1995).

[6] Crippa G., De Lellis C.: Oscillatory solutions to transport equations, Indiana Univ. Math. J. 55, 1-13 (2006).

[7] Da Prato, Giuseppe; Zabczyk, Jerzy, Stochastic equations in infinite dimensions. Encyclopedia of Mathematics and its Applications, 44. Cambridge University Press, Cambridge, 1992.

[8] Davie A. M.: Uniqueness of solutions of stochastic differential equations, Int. Math. Res. Notices 2007, rnm124-126 (2007).

[9] Depauw N.: Non unicité des solutions bornées pour un champ de vecteurs BV en dehors d'un hyperplan, C. R. Math. Acad. Sci. Paris 337, no. 4, 249-252 (2003).

[10] DiPerna, R. J. and Lions, P. L.: Ordinary differential equations, transport theory and Sobolev spaces, Invent. Math. 98, 511-547 (1989). 
[11] Fang, S., Imkeller P., Zhang T.: Global flows for stochastic differential equations without global Lipschitz conditions, Ann. Probab. 35, 180-205 (2007).

[12] Figalli A.: Existence and uniqueness of martingale solutions for SDEs with rough or degenerate coefficients, J. Funct. Anal. 254, 109-153 (2008).

[13] Flandoli F., Russo F., Generalized calculus and SDEs with non regular drift, Stoch. Stoch. Rep. 72 (2002), no. 1-2, 11-54.

[14] Giaquinta M., Modica G., Soucek J.: Cartesian currents in the calculus of variations. I. Cartesian currents, Springer-Verlag, Berlin, 1998.

[15] Gyöngy I., Krylov N. V.: Existence of strong solutions for Itô's stochastic equations via approximations, Probab. Theory Related Fields 105, 143-158 (1996).

[16] Gyöngy I., Shmatkov A.: Rate of convergence of Wong-Zakai approximations for stochastic partial differential equations, Appl. Math. Optim. 54, 315-341 (2006).

[17] Krylov, N. V.: Lectures on elliptic and parabolic equations in Hölder spaces, Graduate Studies in Mathematics, 12. American Mathematical Society, Providence, RI, 1996.

[18] Krylov N. V.: The heat equation in $L_{q}\left((0, T), L_{p}\right)$-spaces with weights, SIAM J. on Math. Anal. 32, 1117-1141 (2001).

[19] Krylov, N. V.: Lectures on elliptic and parabolic equations in Sobolev spaces, 96. American Mathematical Society, Providence, RI, 2008.

[20] Krylov, N. V., Priola, E.: Elliptic and parabolic second-order PDEs with growing coefficients, to appear in Comm. in PDEs, Preprint arXiv.org (2008).

[21] Krylov, N. V., Röckner, M.: Strong solutions of stochastic equations with singular time dependent drift, Probab. Theory Related Fields 131, 154-196 (2005).

[22] Krylov, N. V.; Rozovskii, B. L. Stochastic evolution equations. (Russian) Current problems in mathematics, Vol. 14 (Russian), pp. 71-147, 256, Akad. Nauk SSSR, Vsesoyuz. Inst. Nauchn. i Tekhn. Informatsii, Moscow, 1979.

[23] Kunita, H.: Stochastic differential equations and stochastic flows of diffeomorphisms. Ecole d'été de probabilités de Saint-Flour, XII-1982, 143-303, Lecture Notes in Math. 1097, Springer, Berlin, 1984.

[24] Kunita, H.: First order stochastic partial differential equations. Stochastic analysis (Katata/Kyoto, 1982), 249-269, North-Holland Math. Library, 32, North-Holland, Amsterdam, 1984.

[25] Kunita, H.: Stochastic flows and stochastic differential equations. Cambridge Studies in Advanced Mathematics, 24. Cambridge Univ. Press, Cambridge, 1990.

[26] Ikeda, N. and Watanabe, S.: Stochastic Differential Equations and Diffusion Processes, North Holland-Kodansha, 1981. 
[27] Le Bris C., Lions P. L.: Existence and uniqueness of solutions to Fokker-Planck type equations with irregular coefficients, Comm. Partial Differential Equations 33, 1272-1317 (2008).

[28] Le Jan Y., Raimond O.: Integration of Brownian vector fields, Ann. Probab. 30, 826-873 (2002).

[29] Lions P. L.: Mathematical Topics in Fluid Mechanics. Volume 1: Incompressible models, Oxford Univ. Press, Oxford, 1996.

[30] Majda, A. J., Bertozzi, A. L.: Vorticity and incompressible flow, Cambridge Univ. Press, Cambridge, 2002.

[31] Pardoux E., Equations aux Dérivées Partielles Stochastiques non Linéaires Monotones. Etude de Solutions Fortes de Type Itô, PhD Thesis, Université Paris Sud, 1975.

[32] Protter, P. E.: Stochastic Integration and Differential Equations, Second Edition, Springer, 2004.

[33] Revuz D., Yor M.: Continuous martingales and Brownian motion, Third edition, Springer, Berlin, 1999.

[34] Rozovskii, B. L., Stochastic evolution systems. Linear theory and applications to nonlinear filtering. Translated from the Russian by A. Yarkho. Mathematics and its Applications (Soviet Series), 35. Kluwer Academic Publishers Group, Dordrecht, 1990.

[35] Tessitore G., Zabczyk J.: Wong-Zakai approximations of stochastic evolution equations, J. Evol. Equ. 6, 621-655 (2006).

[36] Veretennikov, Yu. A.: On strong solution and explicit formulas for solutions of stochastic integral equations, Math. USSR Sb. 39, 387-403 (1981).

[37] Zhang, X.: Homeomorphic flows for multi-dimensional SDEs with non-Lipschitz coefficients, Stochastic Processes and their Applications 115, 435-448 (2005).

[38] Zvonkin, A. K. : A transformation of the phase space of a diffusion process that will remove the drift, (Russian) Mat. Sb. (N.S.) 93(135) , 129-149 (1974). 